\title{
Rational Design of Original Fused-cycle Selective Inhibitors of Tryptophan 2,3-Dioxygenase
}

Arina Kozlova ${ }^{1,2,3}$, Léopold Thabault ${ }^{1,4}$, Maxime Liberelle ${ }^{1}$, Simon Klaessens ${ }^{2,3}$, Julien R.C. Prévost ${ }^{1}$, Caroline Mathieu ${ }^{1}$, Luc Pilotte ${ }^{2,3}$, Vincent Stroobant ${ }^{2,3}$, Benoît Van den Eynde ${ }^{2,3,5}$, Raphaël Frédérick ${ }^{1^{*}}$.

1 Louvain Drug Research Institute (LDRI), Université catholique de Louvain (UCLouvain), B-1200 Brussels, Belgium.

${ }^{2}$ Ludwig Institute for Cancer Research, Brussels B-1200, Belgium.

${ }^{3}$ de Duve Institute, UCLouvain, Brussels B-1200, Belgium.

4 Pole of Pharmacology and Therapeutics, Institut de Recherche Expérimentale et Clinique (IREC), UCLouvain,B-1200 Brussels, Belgium.

${ }^{5}$ Walloon Excellence in Life Sciences and Biotechnology, Brussels B-1200, Belgium.

* Correspondence and Lead Contact: Raphaël Frédérick

Mailing address: LDRI, Avenue Mounier 73/B1.73.10, B-1200 Brussels, Belgium.

Phone number: +32 27647341

Email address: raphael.frederick@uclouvain.be 


\section{Content}

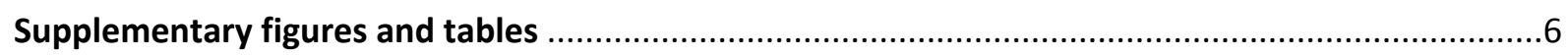

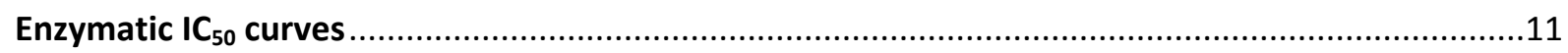

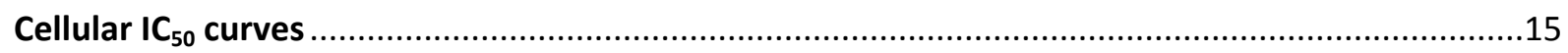

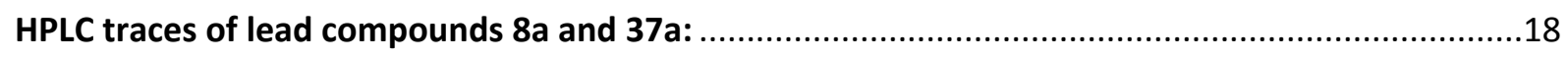

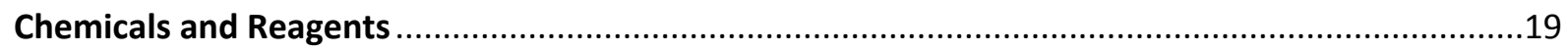

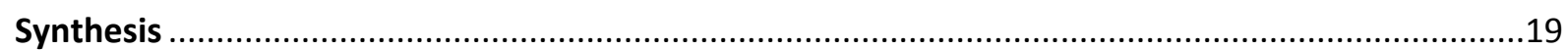

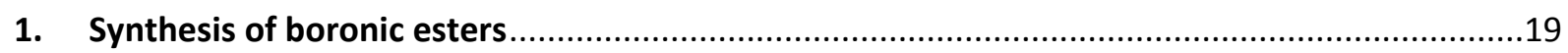

1.1. 1-(phenylsulfonyl)-3-(4,4,5,5-tetramethyl-1,3,2-dioxaborolan-2-yl)-1H-indole (41). ...........19

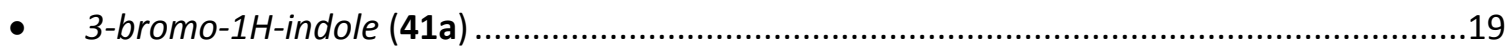

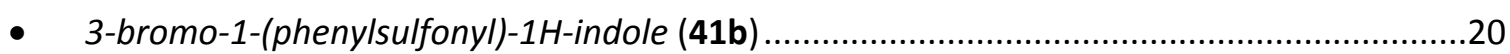

- 1-(phenylsulfonyl)-3-(4,4,5,5-tetramethyl-1,3,2-dioxaborolan-2-yl)-1H-indole (41) ..............20

1.2. tert-butyl 6-fluoro-3-(4,4,5,5-tetramethyl-1,3,2-dioxaborolan-2-yl)-1H-indole-1-carboxylate (42) 20

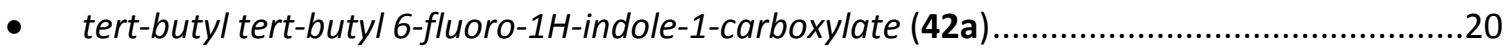

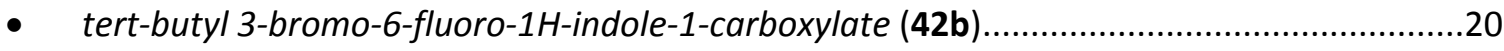

- tert-butyl 6-fluoro-3-(4,4,5,5-tetramethyl-1,3,2-dioxaborolan-2-yl)-1H-indole-1-carboxylate (42) 21

1.3. Synthesis of 1-(Phenylsulfonyl)-3-indolylboronic acid MIDA ester (4-methyl-8-(1(phenylsulfonyl)-1H-indol-3-yl)dihydro-4l4,8l4-[1,3,2]oxazaborolo[2,3-b][1,3,2]oxazaborole2,6(3H,5H)-dione) (43)

2. Synthesis of $\mathbf{N}$-substituted benzotriazoles.

2.1. Bromo-1-(4-methoxybenzyl)-1H-benzo[d][1,2,3]triazole (44) .........................................21

- 6-bromo-1H-benzo[d][1,2,3]triazole (44a)

- 6-bromo-1-(4-methoxybenzyl)-1H-benzo[d][1,2,3]triazole + 5-bromo-1-(4-methoxybenzyl)-

1 -benzo[d][1,2,3]triazole (44)

2.2. Synthesis of $\mathrm{N}$-methylated 6-chloro-1H-benzo[d][1,2,3]triazoles $(\mathbf{7 b} \mathbf{b}, \mathbf{8 b}, \mathbf{9 b})$....................22

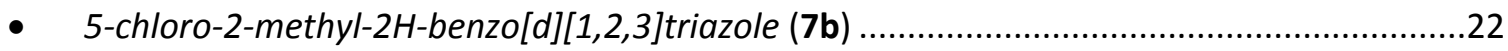

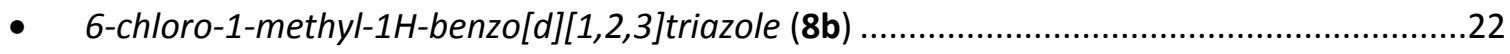

- 5-chloro-1-methyl-1H-benzo[d][1,2,3]triazole (9b) ........................................................22

2.3. 2-(5-chloro-1H-benzo[d][1,2,3]triazol-1-yl)acetonitrile \& 2-(6-chloro-1H-

benzo[d][1,2,3]triazol-1-yl)acetonitrile (21b, 22b)

- While conversion was total, reported yields are low due to purification process. Structures were confirmed with NOESY-NMR and X-ray diffraction crystallography ....................................23

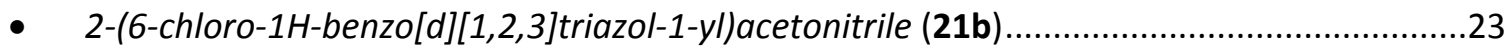

- 2-(5-chloro-1H-benzo[d][1,2,3]triazol-1-yl)acetonitrile (22b)............................................23

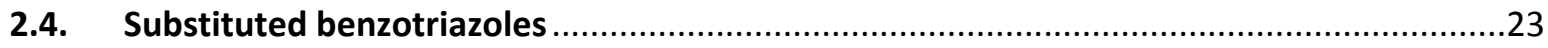


2.4.1. List of $\mathrm{N}$-substituted benzotriazoles obtained starting from 4-Bromo-1-fluoro-2-

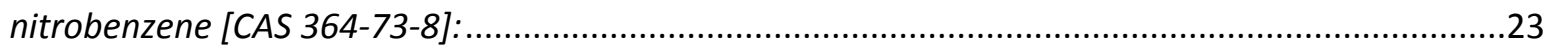

- $\quad$ 5-bromo-1-(2,2,2-trifluoroethyl)-1H-benzo[d][1,2,3]triazole (18d) .....................................23

- $\quad$ 5-bromo-1-(cyclopropylmethyl)-1H-benzo[d][1,2,3]triazole (20d) ......................................24

- 2-(5-bromo-1H-benzo[d][1,2,3]triazol-1-yl)-N,N-dimethylethan-1-amine (26d) ...................24

- 5-bromo-1-((tetrahydrofuran-3-yl)methyl)-1H-benzo[d][1,2,3]triazole (28) .........................25

- 5-bromo-1-((tetrahydrofuran-2-yl)methyl)-1H-benzo[d][1,2,3]triazole (30d) .......................25

- 4-(2-(5-bromo-1H-benzo[d][1,2,3]triazol-1-yl)ethyl)morpholine (32d) .................................26

- tert-butyl 4-((5-bromo-1H-benzo[d][1,2,3]triazol-1-yl)methyl)piperidine-1-carboxylate (34d) 26

- tert-butyl 3-((5-bromo-1H-benzo[d][1,2,3]triazol-1-yl)methyl)pyrrolidine-1-carboxylate (36d) 27

- $\quad$ tert-butyl 4-((5-bromo-1H-benzo[d][1,2,3]triazol-1-yl)methyl)piperazine-1-carboxylate (38d) 27

- 3-(5-bromo-1H-benzo[d][1,2,3]triazol-1-yl)propane-1,2-diol (40d).

2.4.2. List of $\mathrm{N}$-substituted benzotriazoles obtained starting from 4-Bromo-2-fluoro-1nitrobenzene [CAS 364-73-8]:

- 6-bromo-1-(2,2,2-trifluoroethyl)-1H-benzo[d][1,2,3]triazole (17d)....................................28

- 6-bromo-1-(cyclopropylmethyl)-1H-benzo[d][1,2,3]triazole (19d) .......................................29

- 2-(6-bromo-1H-benzo[d][1,2,3]triazol-1-yl)-N,N-dimethylethan-1-amine (25d) ..................29

- 6-bromo-1-((tetrahydrofuran-3-yl)methyl)-1H-benzo[d][1,2,3]triazole (27d) .......................30

- 6-bromo-1-((tetrahydrofuran-2-yl)methyl)-1H-benzo[d][1,2,3]triazole (29d) .......................30

- 4-(2-(6-bromo-1H-benzo[d][1,2,3]triazol-1-yl)ethyl)morpholine (31d) ................................31

- tert-butyl 4-((6-bromo-1H-benzo[d][1,2,3]triazol-1-yl)methyl)piperidine-1-carboxylate (33d) 31

- tert-butyl 3-((6-bromo-1H-benzo[d][1,2,3]triazol-1-yl)methyl)pyrrolidine-1-carboxylate (35d) 32

- tert-butyl 4-((6-bromo-1H-benzo[d][1,2,3]triazol-1-yl)methyl)piperazine-1-carboxylate (37d) 32

- 3-(6-bromo-1H-benzo[d][1,2,3]triazol-1-yl)propane-1,2-diol (39d).

2.5. Synthesis and methylation of 5-Bromotriazolopyridine.

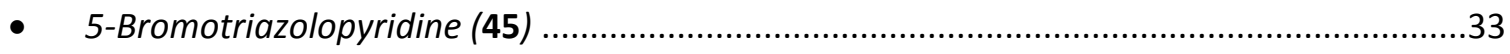

- 6-bromo-1-methyl-1H-[1,2,3]triazolo[4,5-b]pyridine (14b) .................................................

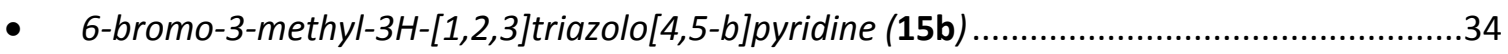

- 6-bromo-2-methyl-2H-[1,2,3]triazolo[4,5-b]pyridine (13b) ..................................................

2.6. General procedures of nitration (A) and reduction (B) of methylated 5- 


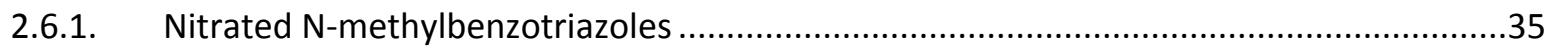

- $\quad$ 5-chloro-2-methyl-4-nitro-2H-benzo[d][1,2,3]triazole (10b) ..............................................

- $\quad$ 5-chloro-2-methyl-4, 6-dinitro-2H-benzo[d][1,2,3]triazole ................................................. 35

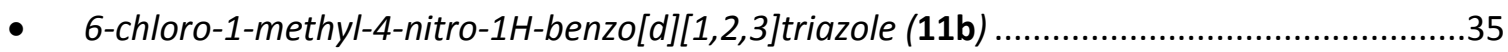

- $\quad$ 5-chloro-1-methyl-4 nitro-1H-benzo[d][1,2,3]triazole (12b) ...............................................

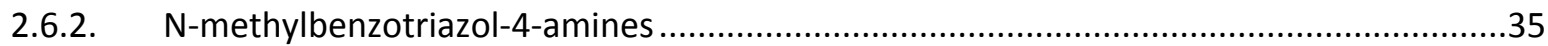

- $\quad$ 5-chloro-2-methyl-2H-benzo[d][1,2,3]triazol-4-amine (10c). ..............................................

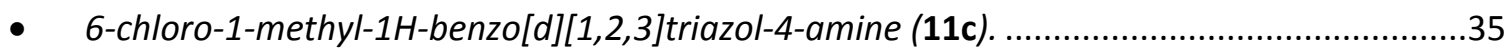

- 5-chloro-1-methyl-1H-benzo[d][1,2,3]triazol-4-amine (12c). ...............................................35

2.7. General procedures for the Suzuki-Miyaura cross-coupling reaction ................................36

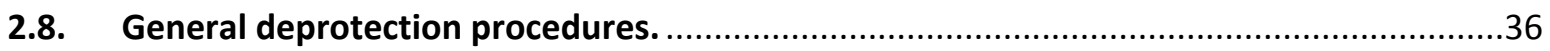

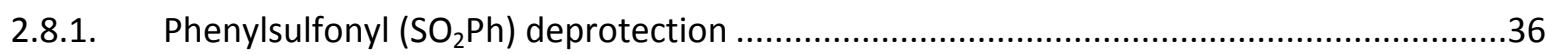

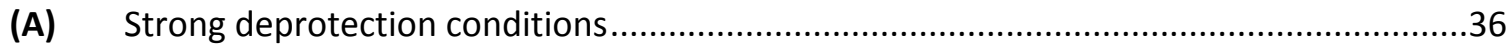

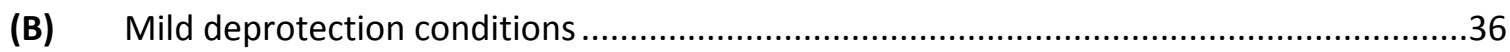

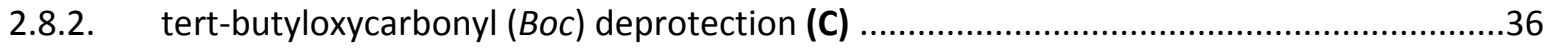

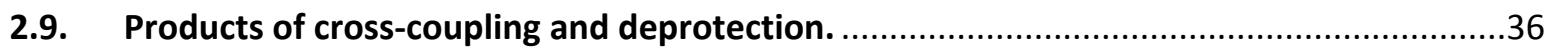

2.9.1. List of compounds obtained following cross-coupling procedure $A$ (using 43 )................36

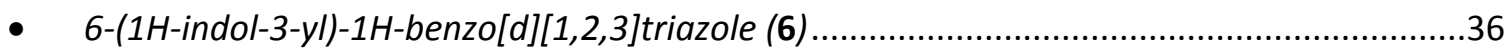

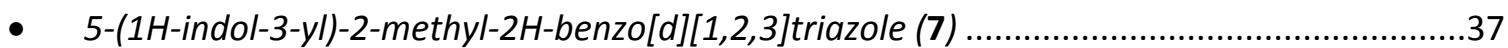

- $\quad$ 5-(1H-indol-3-yl)-2-methyl-2H-benzo[d][1,2,3]triazol-4-amine (10).....................................37

- $\quad$ 6-(1H-indol-3-yl)-1-methyl-1H-benzo[d][1,2,3]triazole (8) ................................................38

- $\quad$ 6-(1H-indol-3-yl)-1-methyl-1H-benzo[d][1,2,3]triazol-7-amine (11)....................................38

- $\quad$ 5-(1H-indol-3-yl)-1-methyl-1H-benzo[d][1,2,3]triazole (9) ..................................................39

- $\quad$ 5-(1H-indol-3-yl)-1-methyl-4-nitro-1H-benzo[d][1,2,3]triazole (16) ....................................40

- $\quad$ 5-(1H-indol-3-yl)-1-methyl-1H-benzo[d][1,2,3]triazol-4-amine (12) ......................................40

- 2-(6-(1H-indol-3-yl)-1H-benzo[d][1,2,3]triazol-1-yl)acetamide (21) .....................................41

- 2-(6-(1H-indol-3-yl)-1H-benzo[d][1,2,3]triazol-1-yl)acetic acid (23) ....................................41

- 2-(5-(1H-indol-3-yl)-1H-benzo[d][1,2,3]triazol-1-yl)acetamide (22) .....................................41

- 2-(5-(1H-indol-3-yl)-1H-benzo[d][1,2,3]triazol-1-yl)acetic acid (24) ....................................42

2.12.2 List of compounds obtained following cross-coupling general procedure B (using 41) ........42

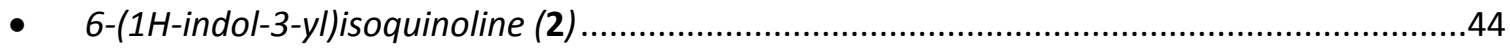

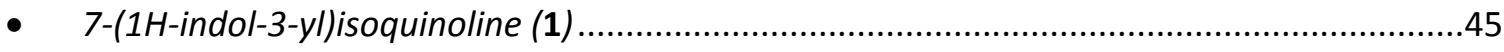

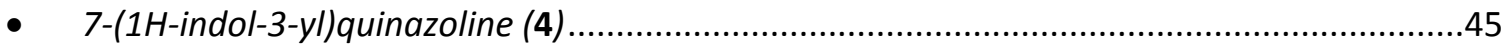

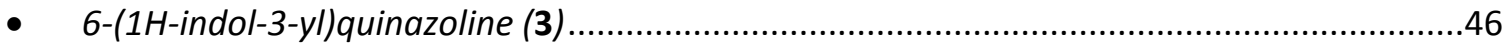

- 6-(1H-indol-3-yl)-3,4-dihydroquinazolin-2(1H)-one (5) ........................................................46 
- 1-(cyclopropylmethyl)-6-(1H-indol-3-yl)-1H-benzo[d][1,2,3]triazole (19)

- 1-(cyclopropylmethyl)-5-(1H-indol-3-yl)-1H-benzo[d][1,2,3]triazole (20).

- 2-(6-(1H-indol-3-yl)-1H-benzo[d][1,2,3]triazol-1-yl)-N,N-dimethylethan-1-amine (25)

- 2-(5-(1H-indol-3-yl)-1H-benzo[d][1,2,3]triazol-1-yl)-N,N-dimethylethan-1-amine (26). .48

- 6-(1H-indol-3-yl)-1-((tetrahydrofuran-3-yl)methyl)-1H-benzo[d][1,2,3]triazole (27) .48

- 5-(1H-indol-3-yl)-1-((tetrahydrofuran-3-yl)methyl)-1H-benzo[d][1,2,3]triazole (28) . .49

- 6-(1H-indol-3-yl)-1-((tetrahydrofuran-2-yl)methyl)-1H-benzotriazole (29) .49

- 5-(1H-indol-3-yl)-1-((tetrahydrofuran-2-yl)methyl)-1H-benzotriazole (30) .50

- 4-(2-(6-(1H-indol-3-yl)-1H-benzotriazol-1-yl)ethyl)morpholine (31) .51

- 4-(2-(5-(1H-indol-3-yl)-1H-benzotriazol-1-yl)ethyl)morpholine (32)

- 3-(6-(1H-indol-3-yl)-1H-benzo[d][1,2,3]triazol-1-yl)propane-1,2-diol (39)

- 3-(5-(1H-indol-3-yl)-1H-benzo[d][1,2,3]triazol-1-yl)propane-1,2-diol (40)

2.9.2. List of compounds obtained following cross-coupling procedure $B$.

A. using tert-butyl 6-fluoro-3-(4,4,5,5-tetramethyl-1,3,2-dioxaborolan-2-yl)-1H-indole-1carboxylate (42).

- 6-(6-fluoro-1H-indol-3-yl)-1-methyl-1H-benzotriazole (8a)

- 6-(6-fluoro-1H-indol-3-yl)-1-(piperazin-1-ylmethyl)-1H-benzo[d][1,2,3]triazole (37a) .53

B. Using tert-butyl 3-(4,4,5,5-tetramethyl-1,3,2-dioxaborolan-2-yl)-1H-indole-1-carboxylate........53

- 6-(1H-indol-3-yl)-1-(2,2,2-trifluoroethyl)-1H-benzo[d][1,2,3]triazole (17)........................53

- 6-(1H-indol-3-yl)-1-(2,2,2-trifluoroethyl)-1H-benzo[d][1,2,3]triazole (18).........................54

- 6-(1H-indol-3-yl)-1-(piperidin-4-ylmethyl)-1H-benzo[d][1,2,3]triazole (34)........................54

- $\quad$ 5-(1H-indol-3-yl)-1-(piperidin-4-ylmethyl)-1H-benzo[d][1,2,3]triazole (34)........................55

- 6-(1H-indol-3-yl)-1-(pyrrolidin-3-ylmethyl)-1H-benzo[d][1,2,3]triazole (35) ........................56

- 5-(1H-indol-3-yl)-1-(pyrrolidin-3-ylmethyl)-1H-benzo[d][1,2,3]triazole (36) ......................56

- $\quad$ 6-(1H-indol-3-yl)-1-(2-(piperazin-1-yl)ethyl)-1H-benzo[d][1,2,3]triazole (37) .....................57

- 5-(1H-indol-3-yl)-1-(piperazin-4-yl)ethyl)-1H-benzo[d][1,2,3]triazole (38) .57 


\section{Supplementary figures and tables}

Table S1. Inhibitory affinities (confidence intervals $95 \% \mathrm{IC}_{50}$ ) of compounds against hTDO2 in the spectrophotometric assay and P815B cells.

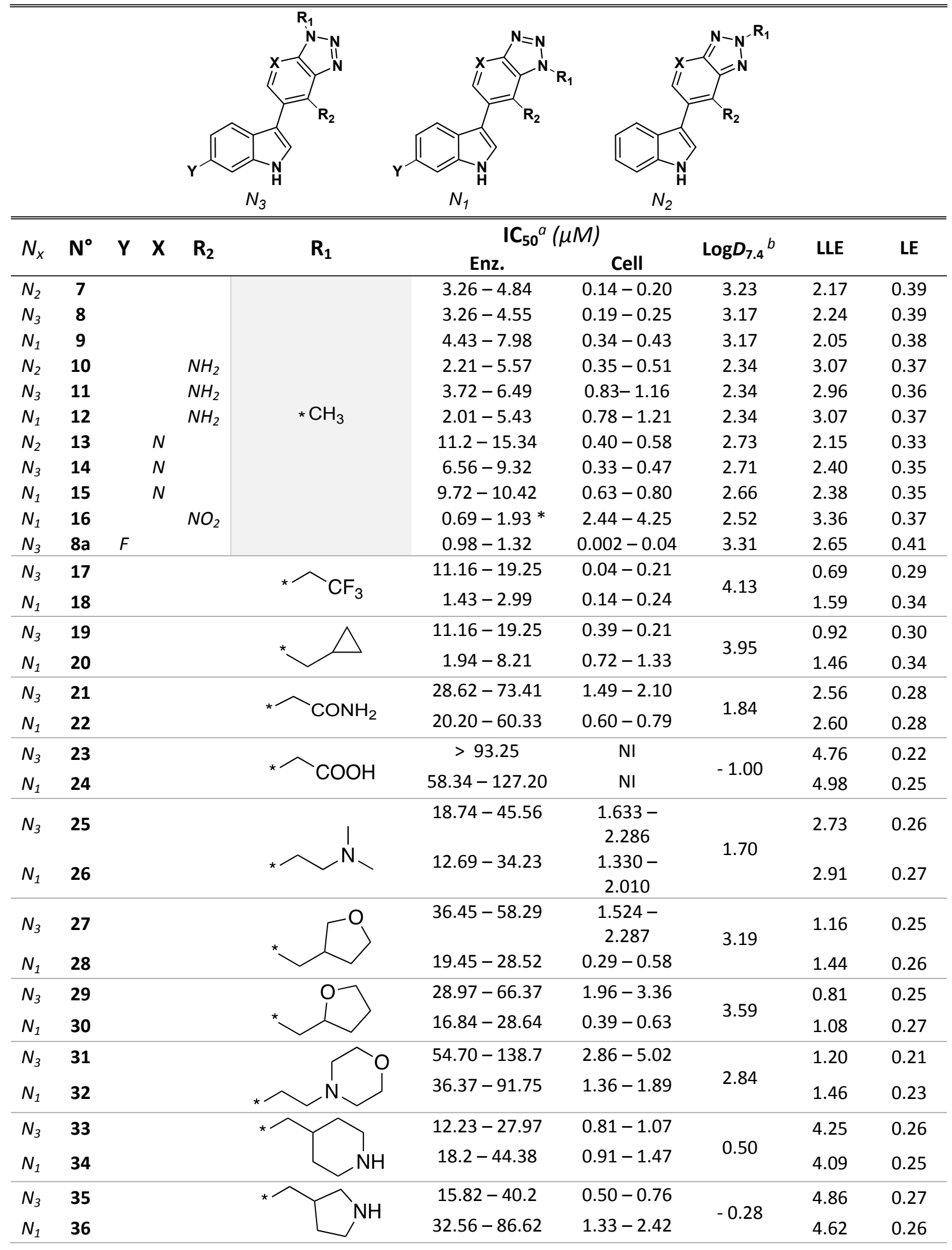




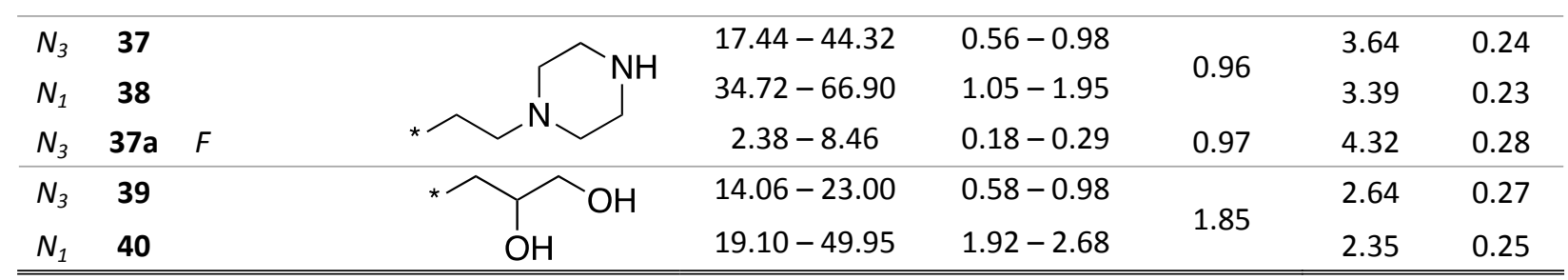




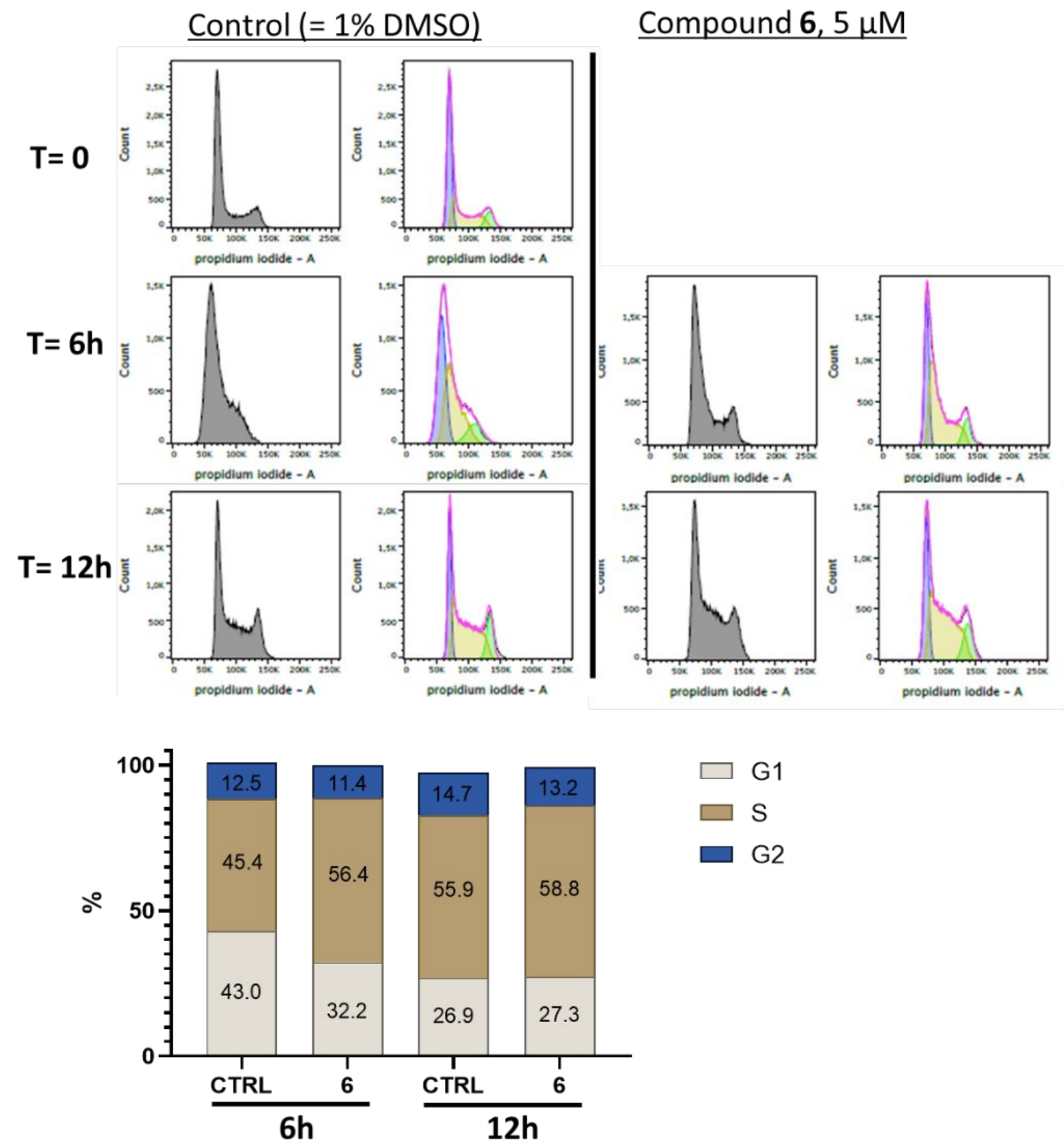

Figure S1. Evaluation of influence of compound 6 (5-(1H-indol-3-yl)-1H-benzo[d] [1,2,3]triazole) on P815B cell cycle. Cell cycle phase distribution was determined at $6 \mathrm{~h}$ and $12 \mathrm{~h}$ by flow-cytometry. Bar diagram represents the distribution of cells in different phases of the cell cycle. 


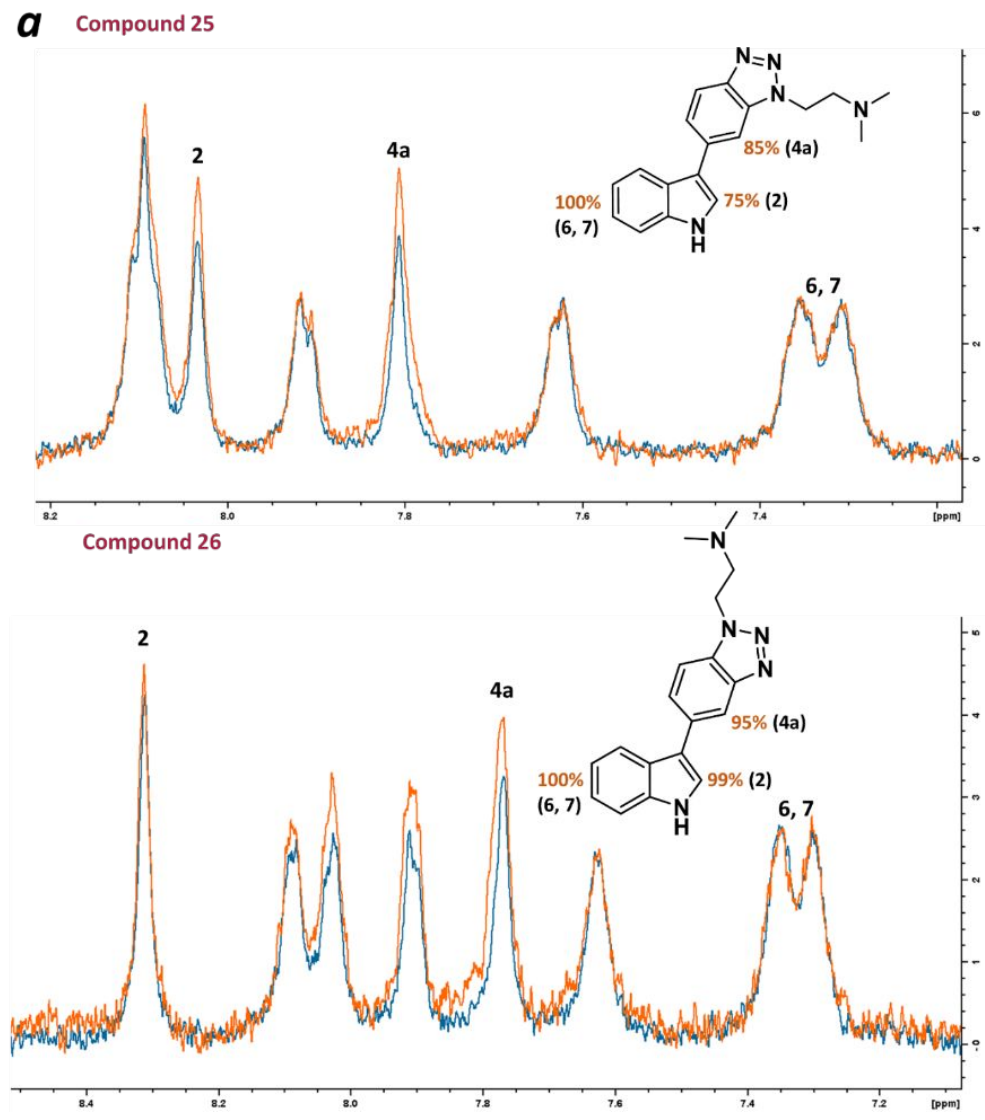

b compound 35

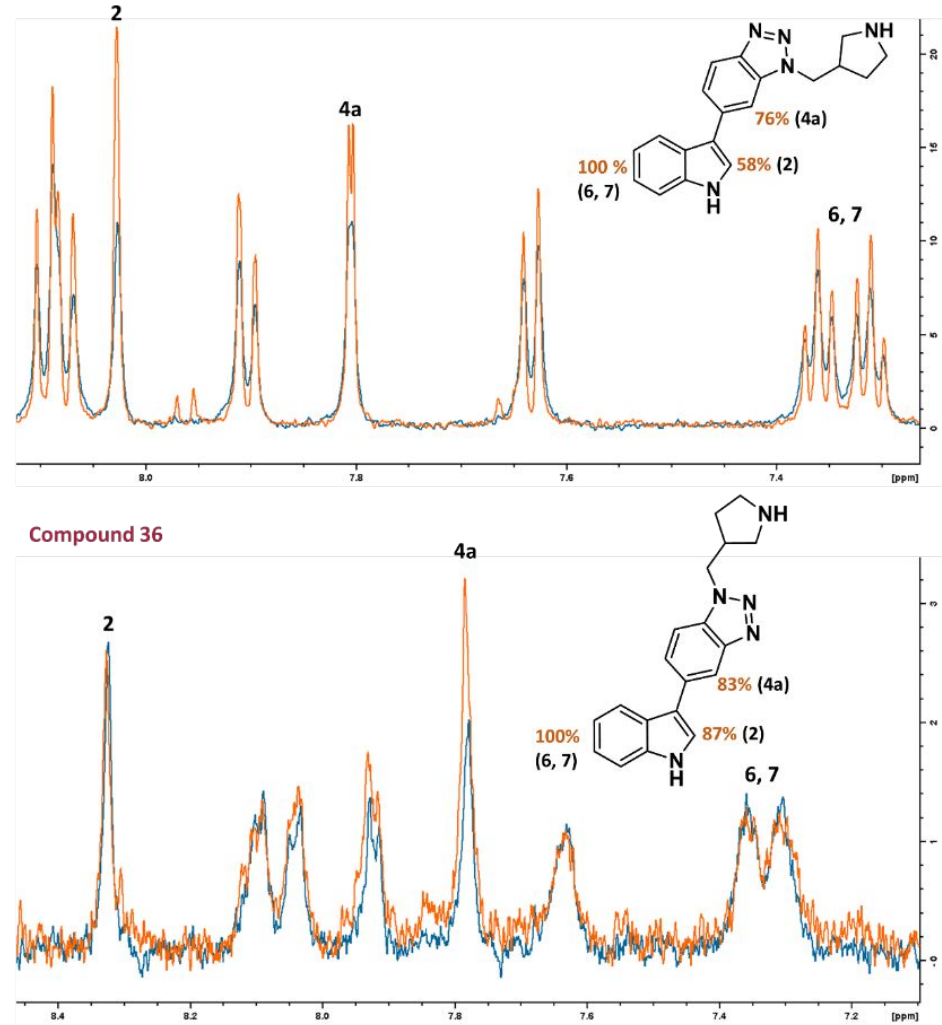

Figure S2. Epitope mapping of regioisomers 26, 27 (a) and 36, 37 (b). 
$a$

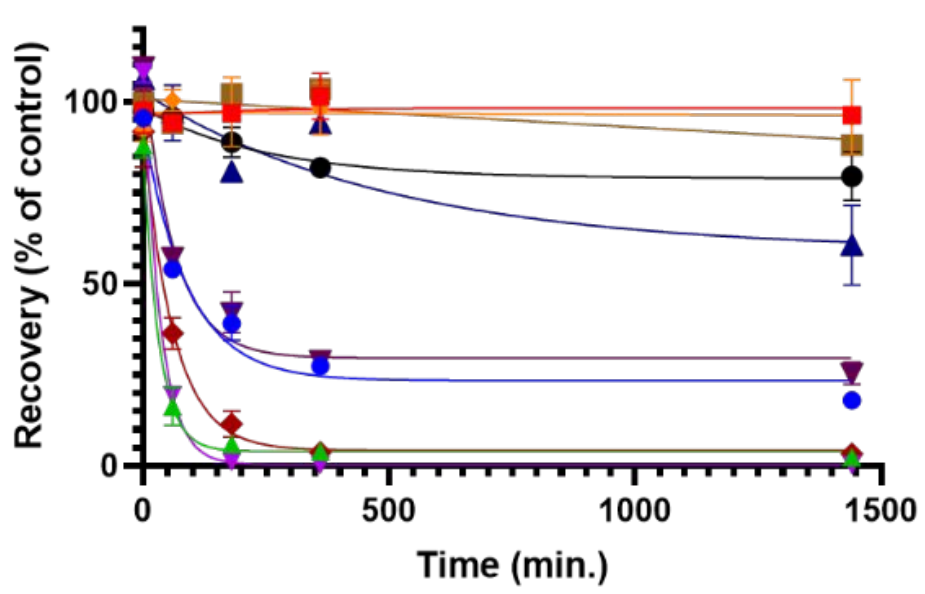

- LM10

$-6$

$\rightarrow 8$

$+8 a$

$\rightarrow 18$

- 26

-35
$-\quad 37$

$-\quad 37$

- $37 a$

- 38

b

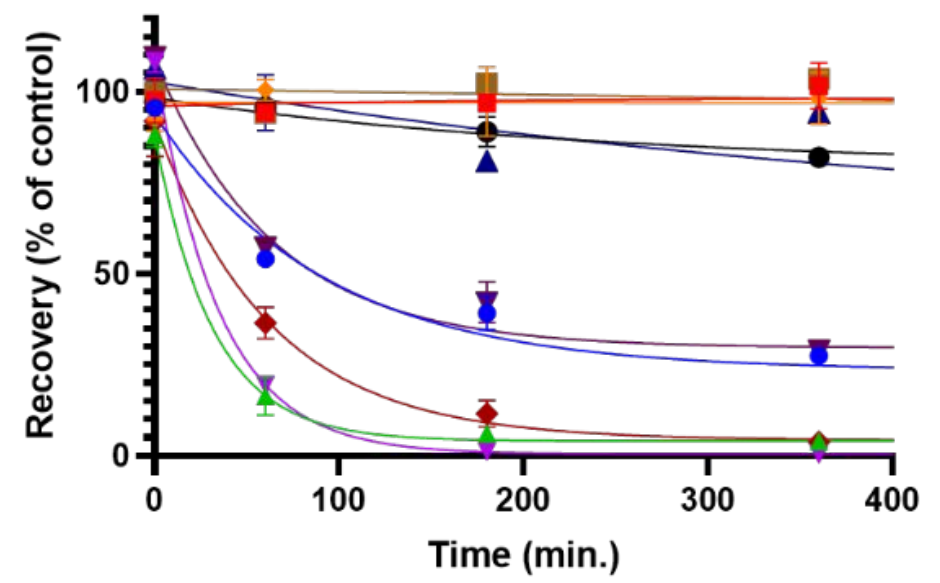

Figure S3. Microsomal stability, half-life curves. (a) Recovery of the different studied compounds over time in the presence of microsomal extracts. (b) Closeup view on the $0-360$ minutes portion of the curves. The plotted values are the result of two independent experiments. 


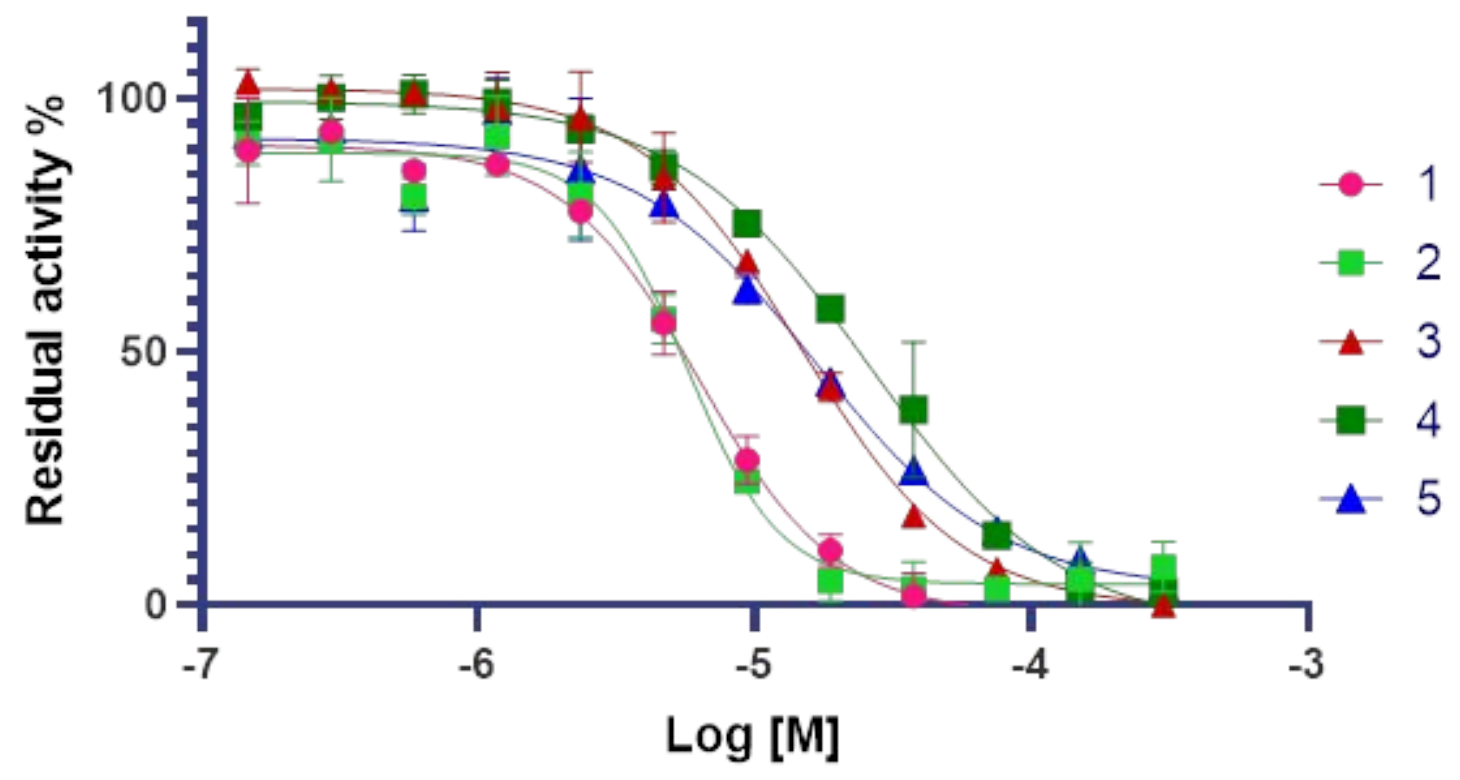

Inhibition curves of compounds $\mathbf{1}$ to $\mathbf{5}$ in enzymatic assays. All experiments were performed in triplicates, in at least two separate experiments.

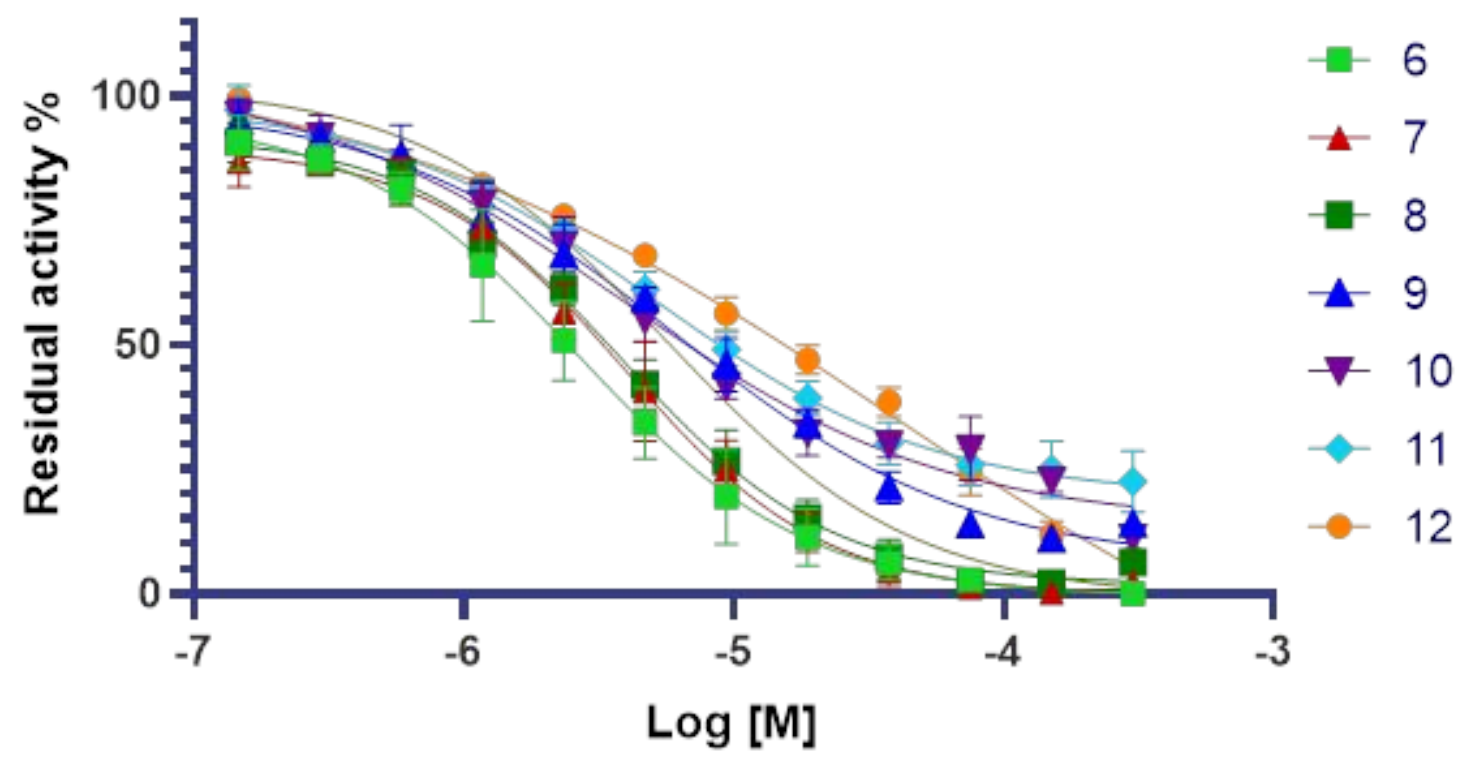

Inhibition curves of compounds $\mathbf{6}$ to $\mathbf{1 2}$ in enzymatic assays. All experiments were performed in triplicates, in at least two separate experiments. 


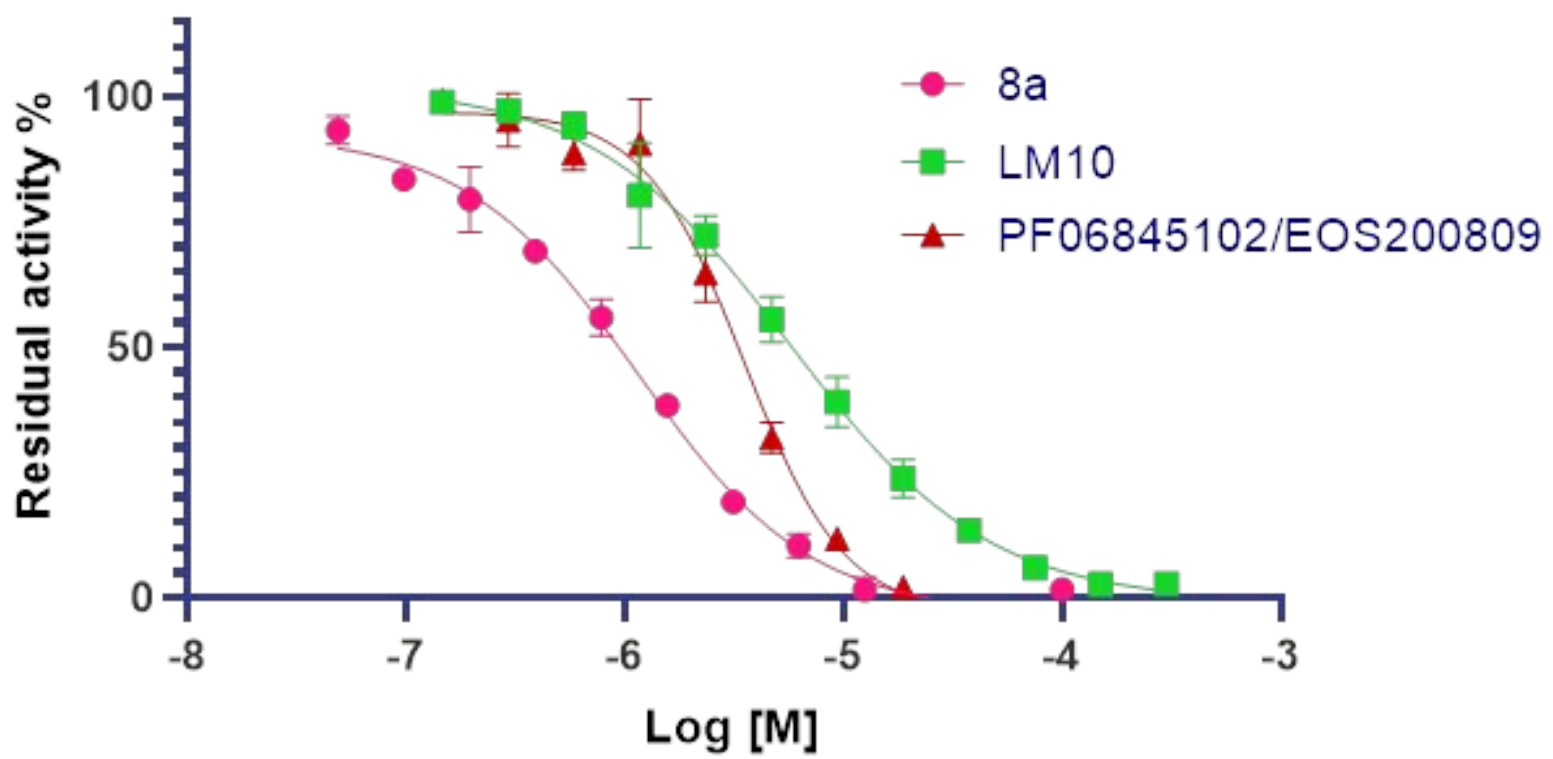

Inhibition curves of compounds 8a, LM10, and PF06845102/EOS200809 in enzymatic assays. All experiments were performed in triplicates, in at least two separate experiments.

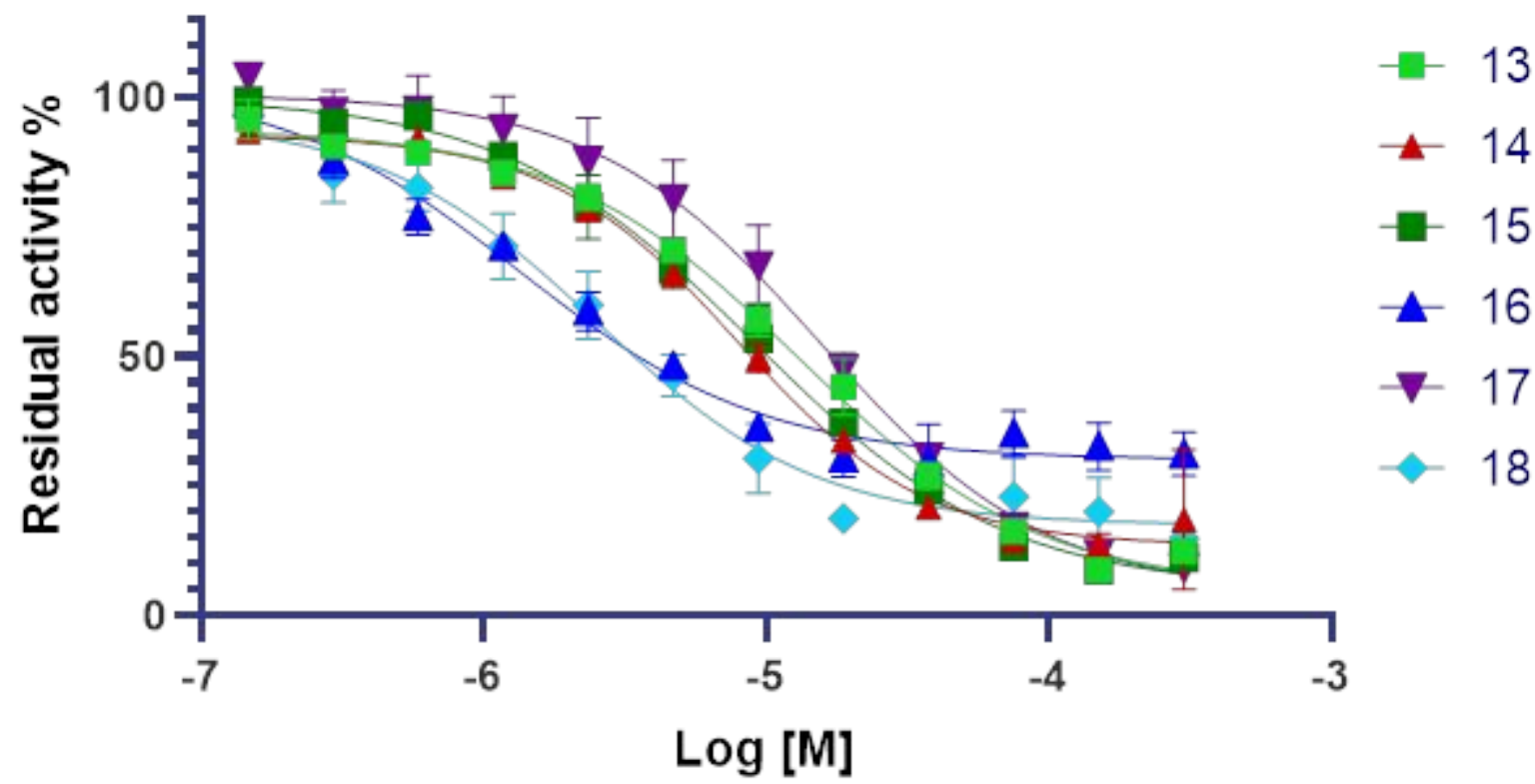

Inhibition curves of compounds $\mathbf{1 3}$ to $\mathbf{1 8}$ in enzymatic assays. All experiments were performed in triplicates, in at least two separate experiments. 


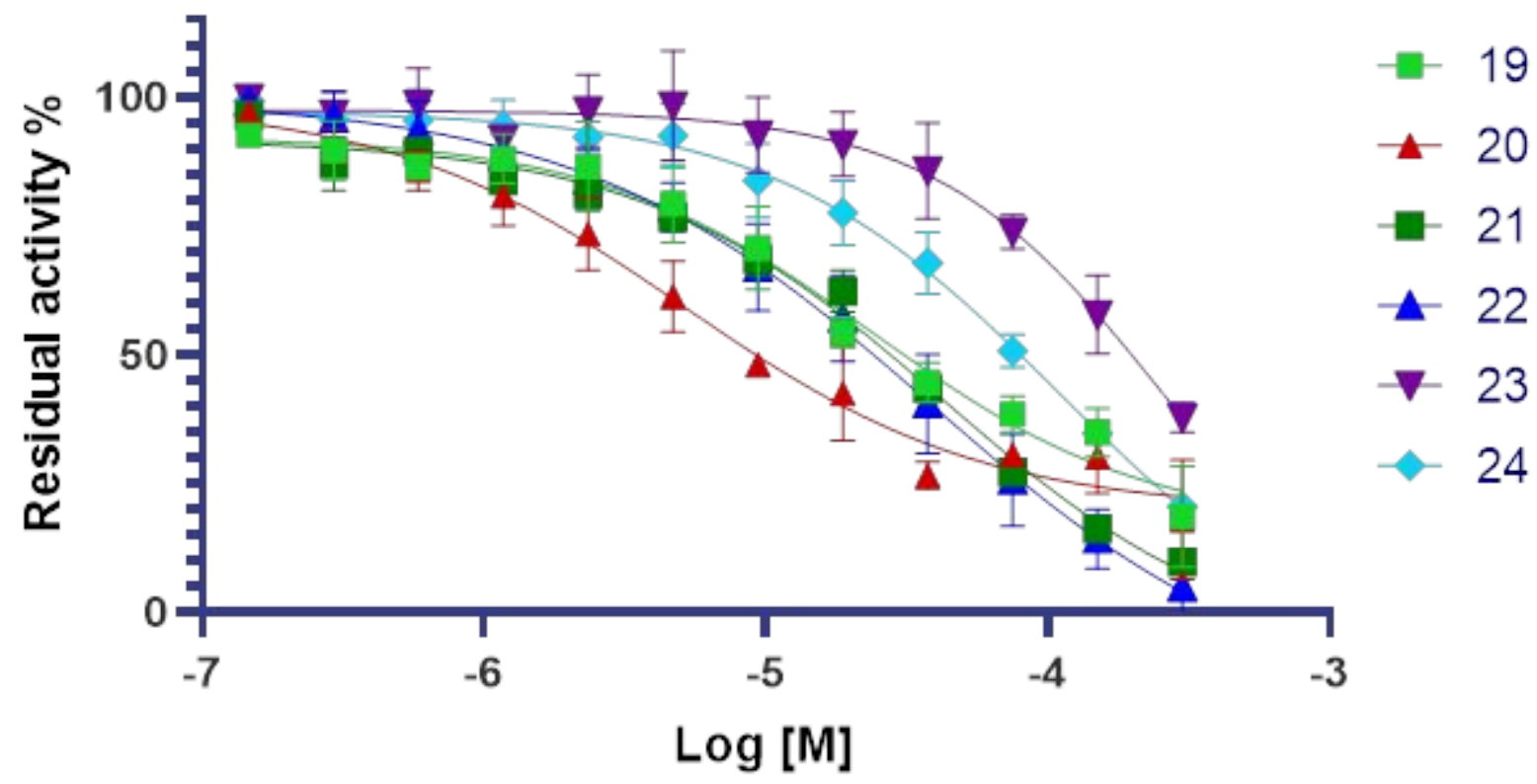

Inhibition curves of compounds $\mathbf{1 9}$ to $\mathbf{2 4}$ in enzymatic assays. All experiments were performed in triplicates, in at least two separate experiments.

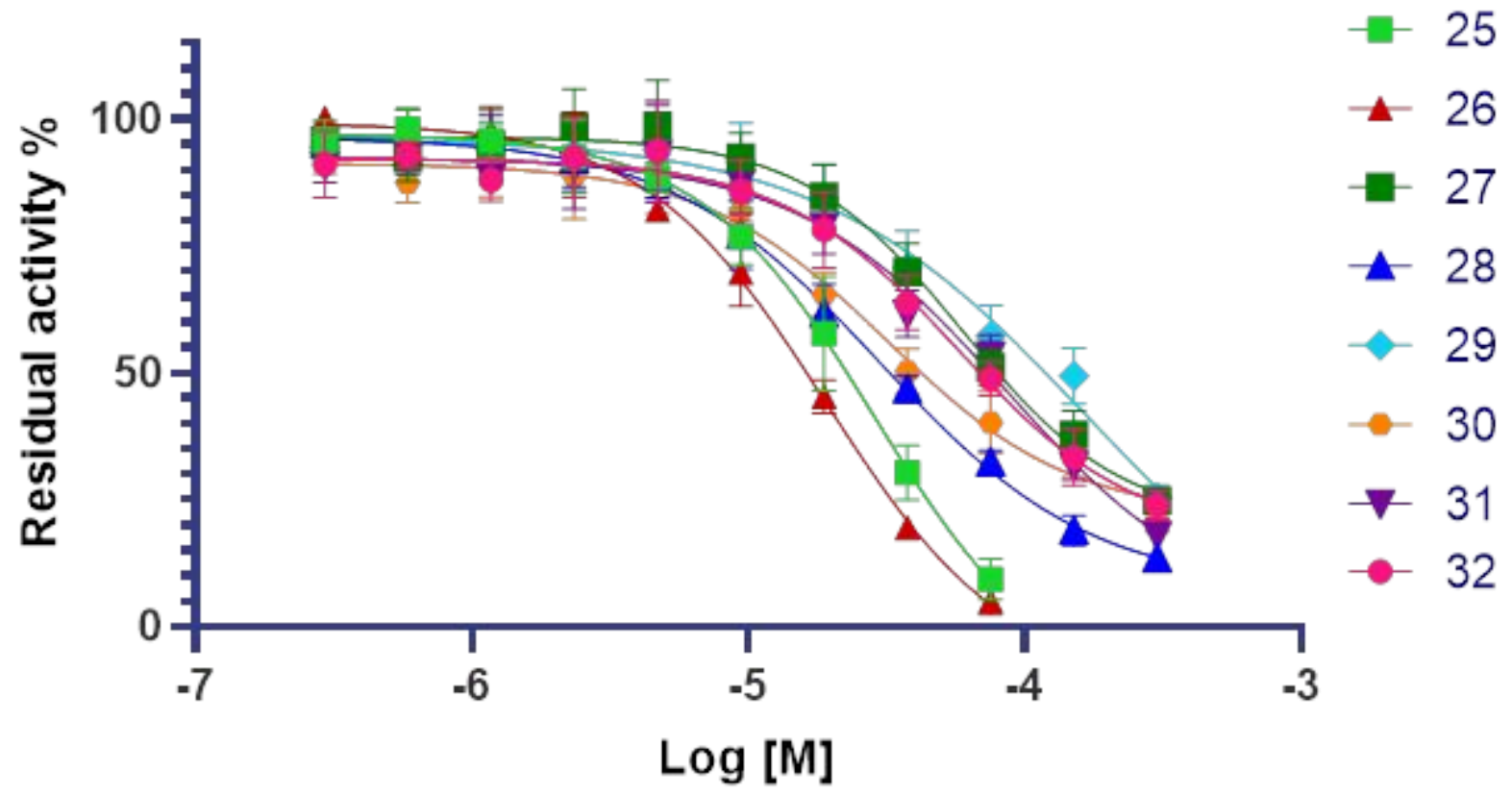

Inhibition curves of compounds $\mathbf{2 5}$ to $\mathbf{3 2}$ in enzymatic assays. All experiments were performed in triplicates, in at least two separate experiments. 


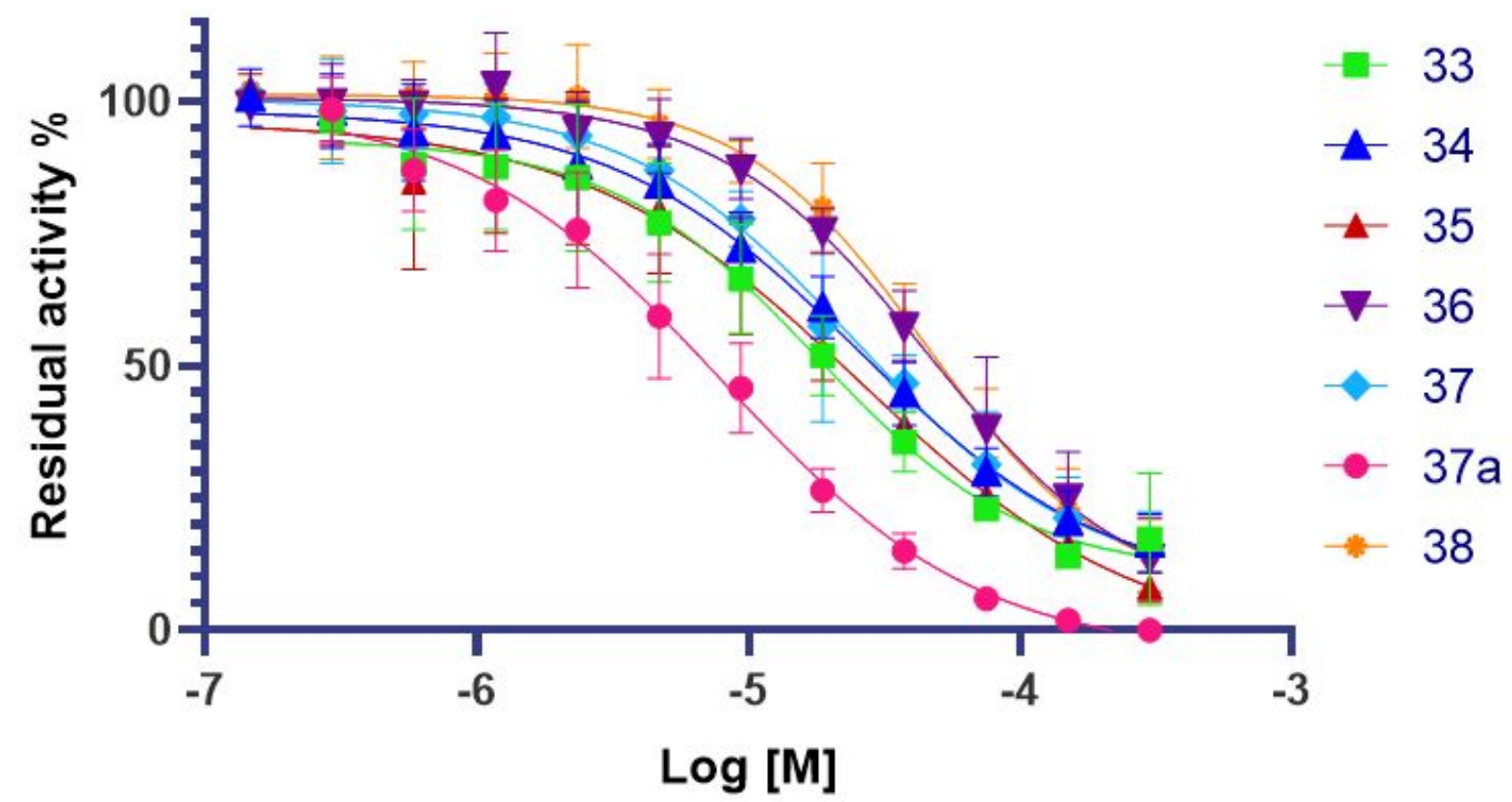

Inhibition curves of compounds $\mathbf{3 3}$ to $\mathbf{3 8}$ in enzymatic assays. All experiments were performed in triplicates, in at least two separate experiments.

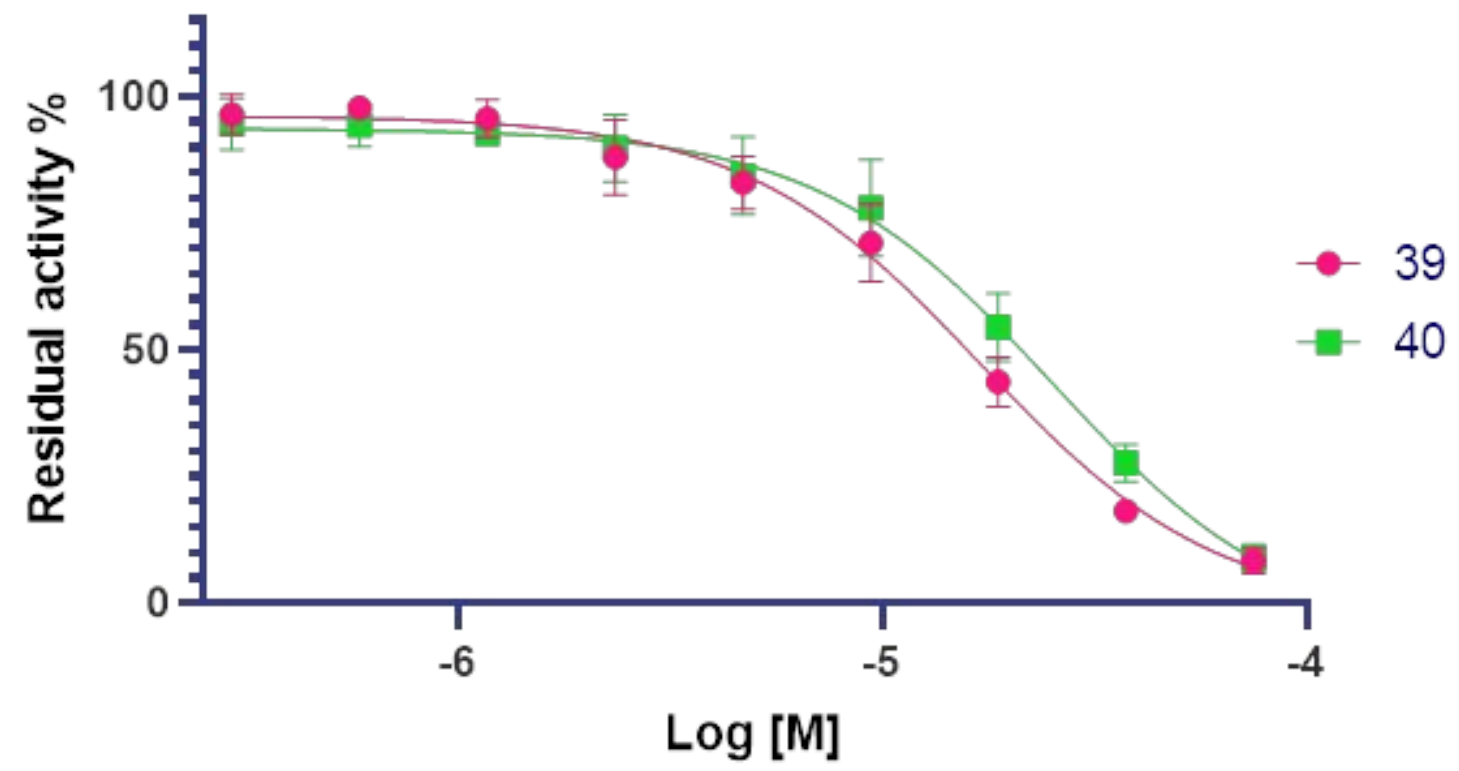

Inhibition curves of compounds $\mathbf{3 9}$ to $\mathbf{4 0}$ in enzymatic assays. All experiments were performed in triplicates, in at least two separate experiments. 


\section{Cellular IC 50 curves}

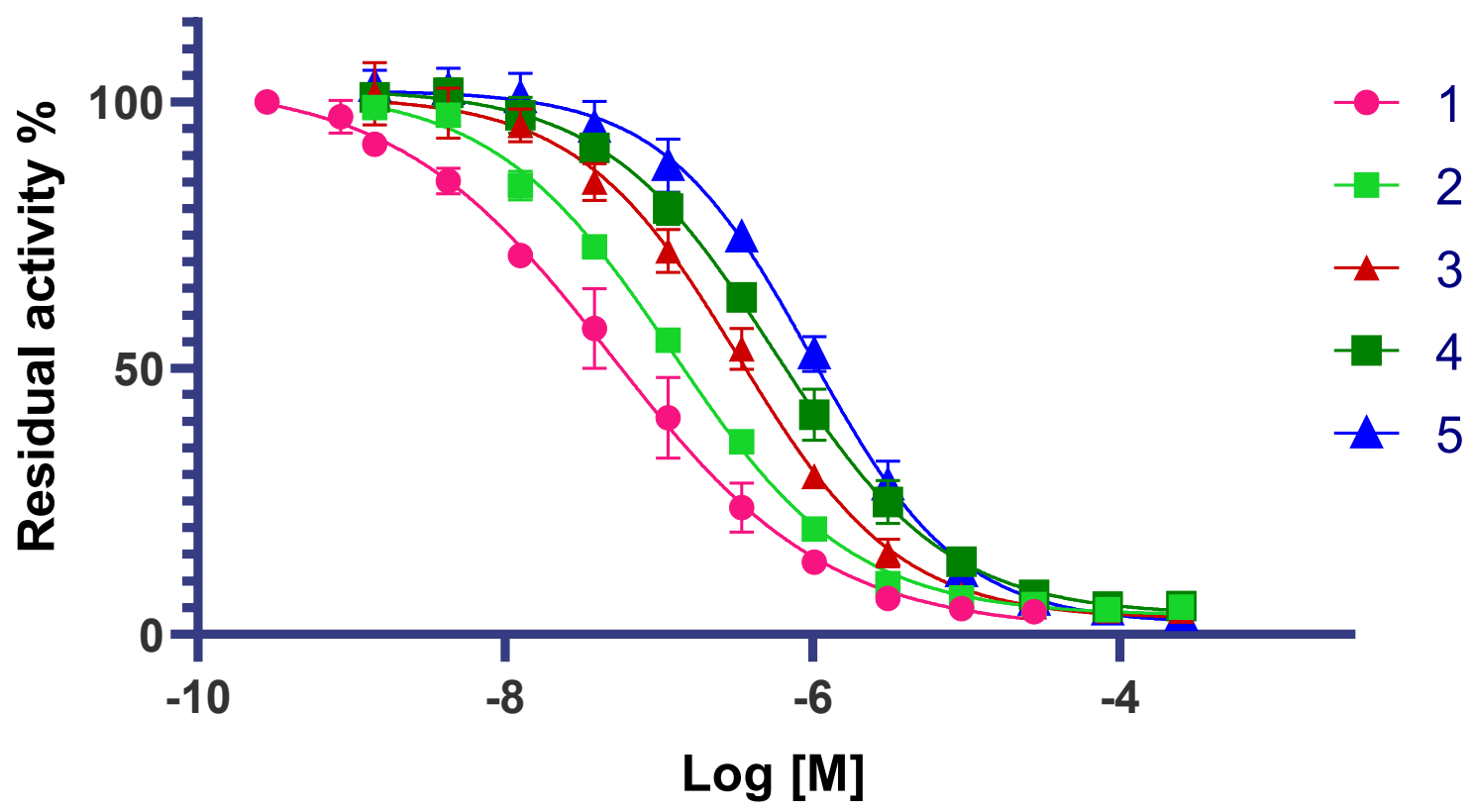

Inhibition curves of compounds $\mathbf{1}$ to $\mathbf{5}$ in cellular assays. All experiments were performed in triplicates, in at least two separate experiments.

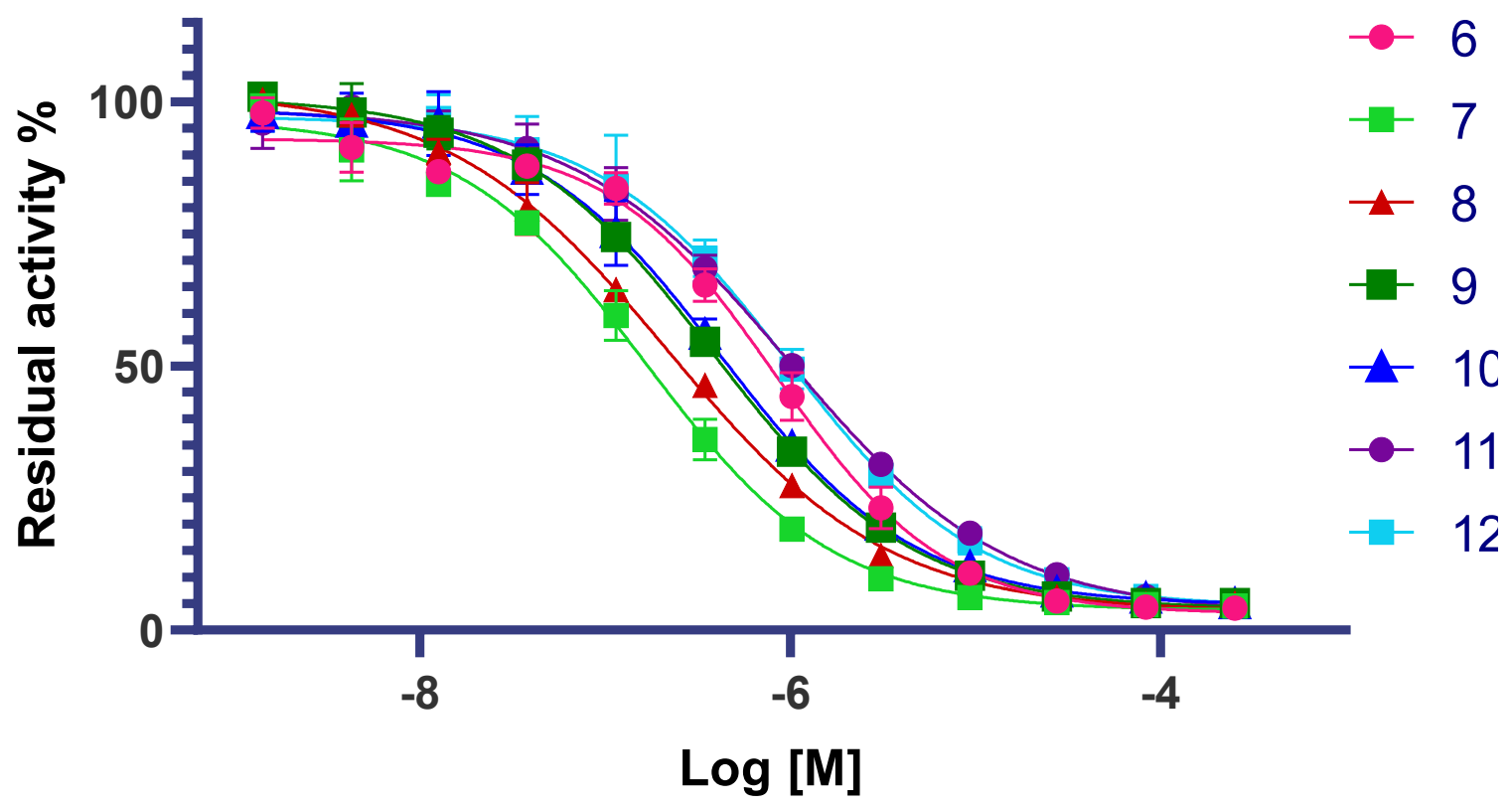

Inhibition curves of compounds $\mathbf{6}$ to $\mathbf{1 2}$ in cellular assays. All experiments were performed in triplicates, in at least two separate experiments. 


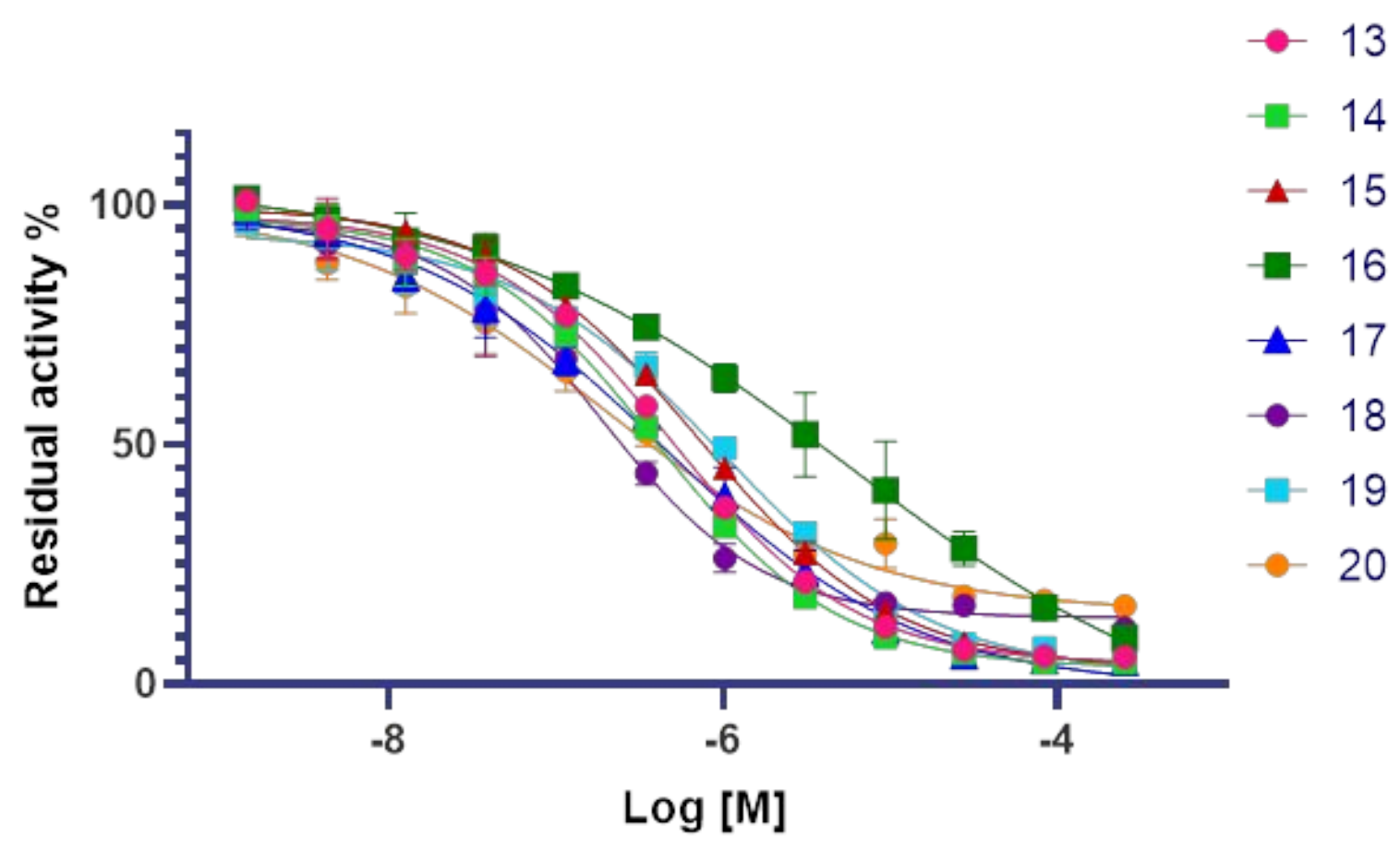

Inhibition curves of compounds $\mathbf{1 3}$ to $\mathbf{2 0}$ in cellular assays. All experiments were performed in triplicates, in at least two separate experiments.

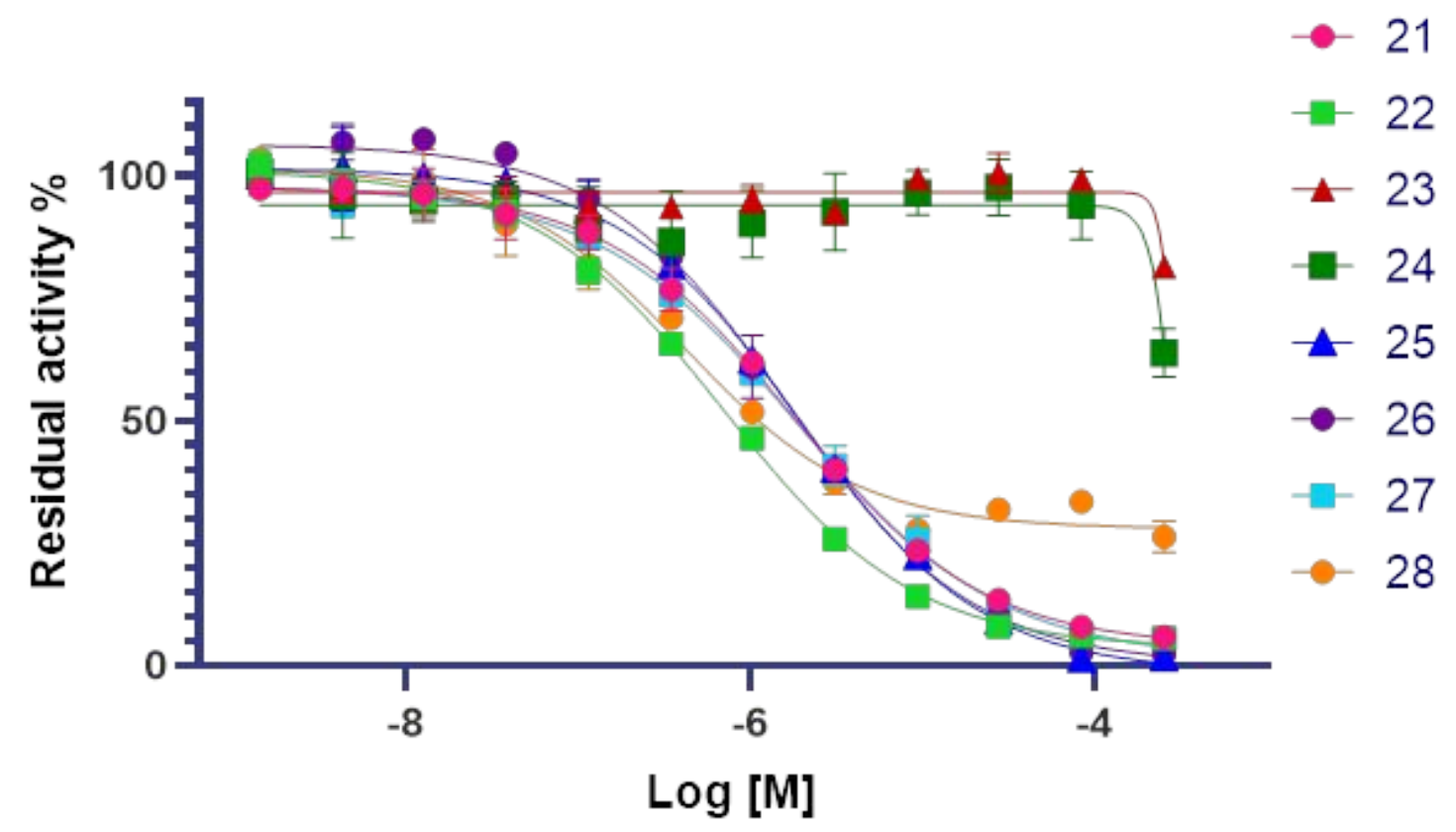

Inhibition curves of compounds $\mathbf{2 1}$ to $\mathbf{2 8}$ in cellular assays. All experiments were performed in triplicates, in at least two separate experiments. 


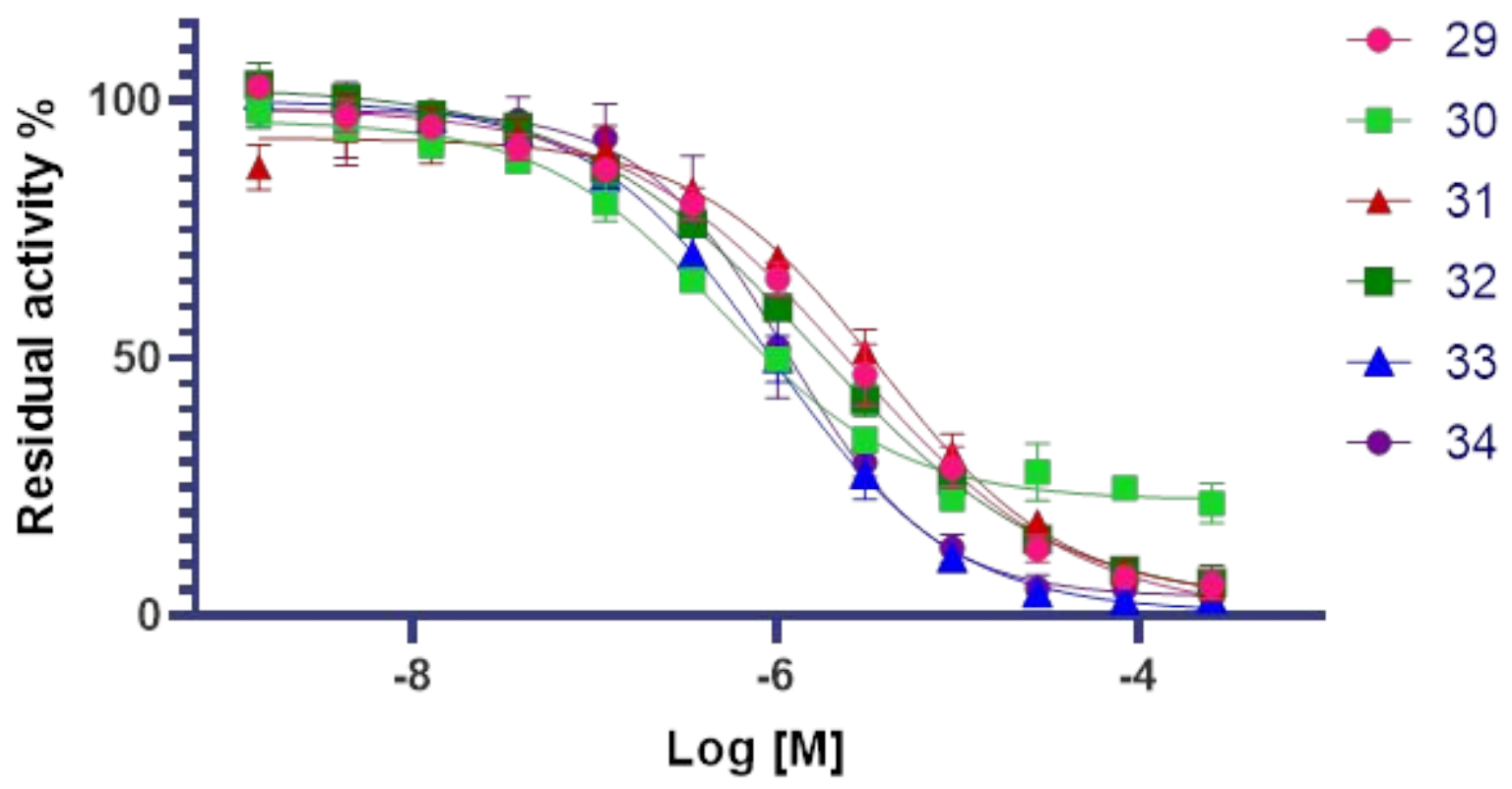

Inhibition curves of compounds $\mathbf{2 9}$ to $\mathbf{3 4}$ in cellular assays. All experiments were performed in triplicates, in at least two separate experiments.

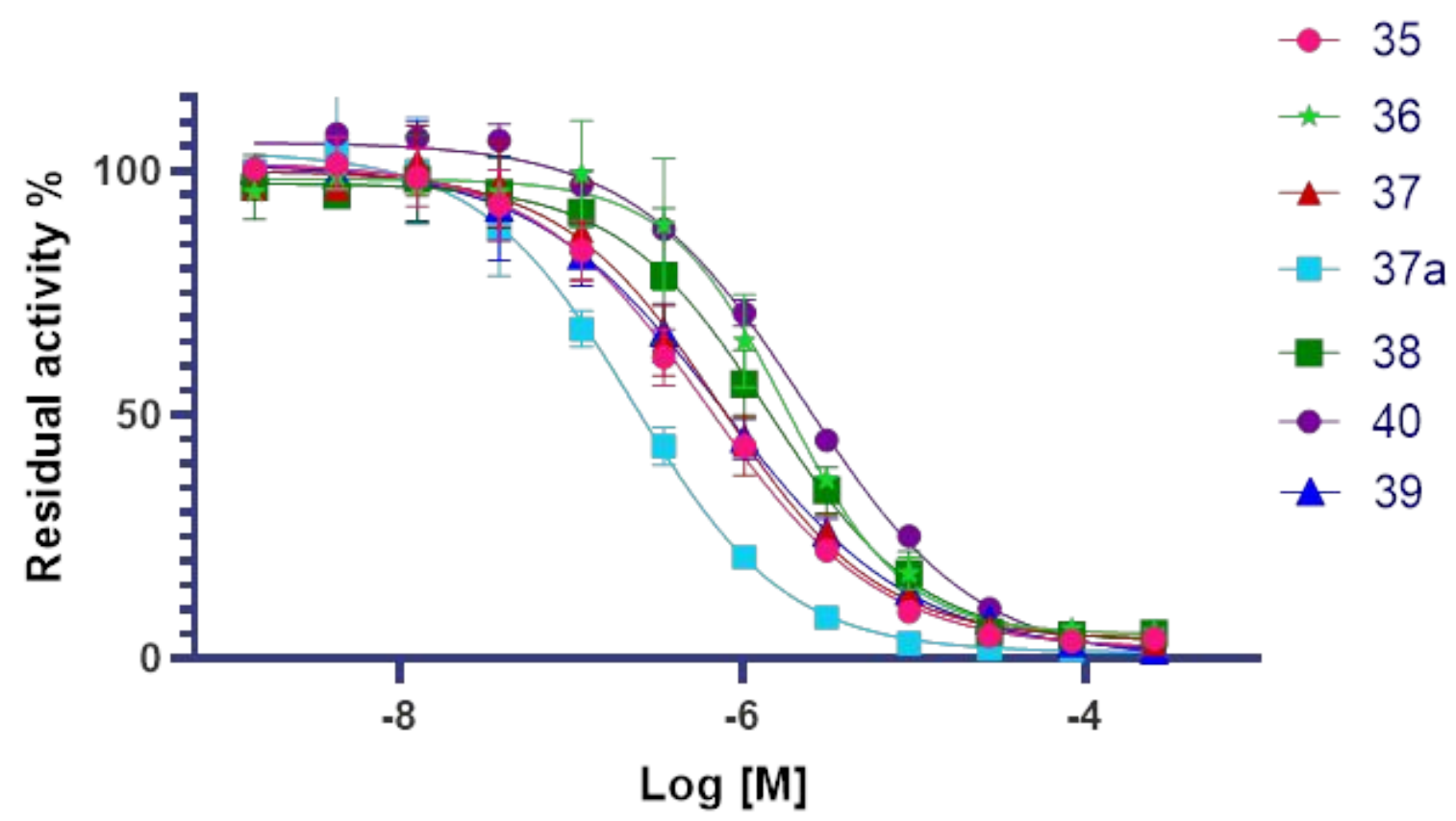

Inhibition curves of compounds $\mathbf{3 5}$ to $\mathbf{4 0}$ in cellular assays. All experiments were performed in triplicates, in at least two separate experiments. 
HPLC traces of lead compounds $8 a$ and 37a:

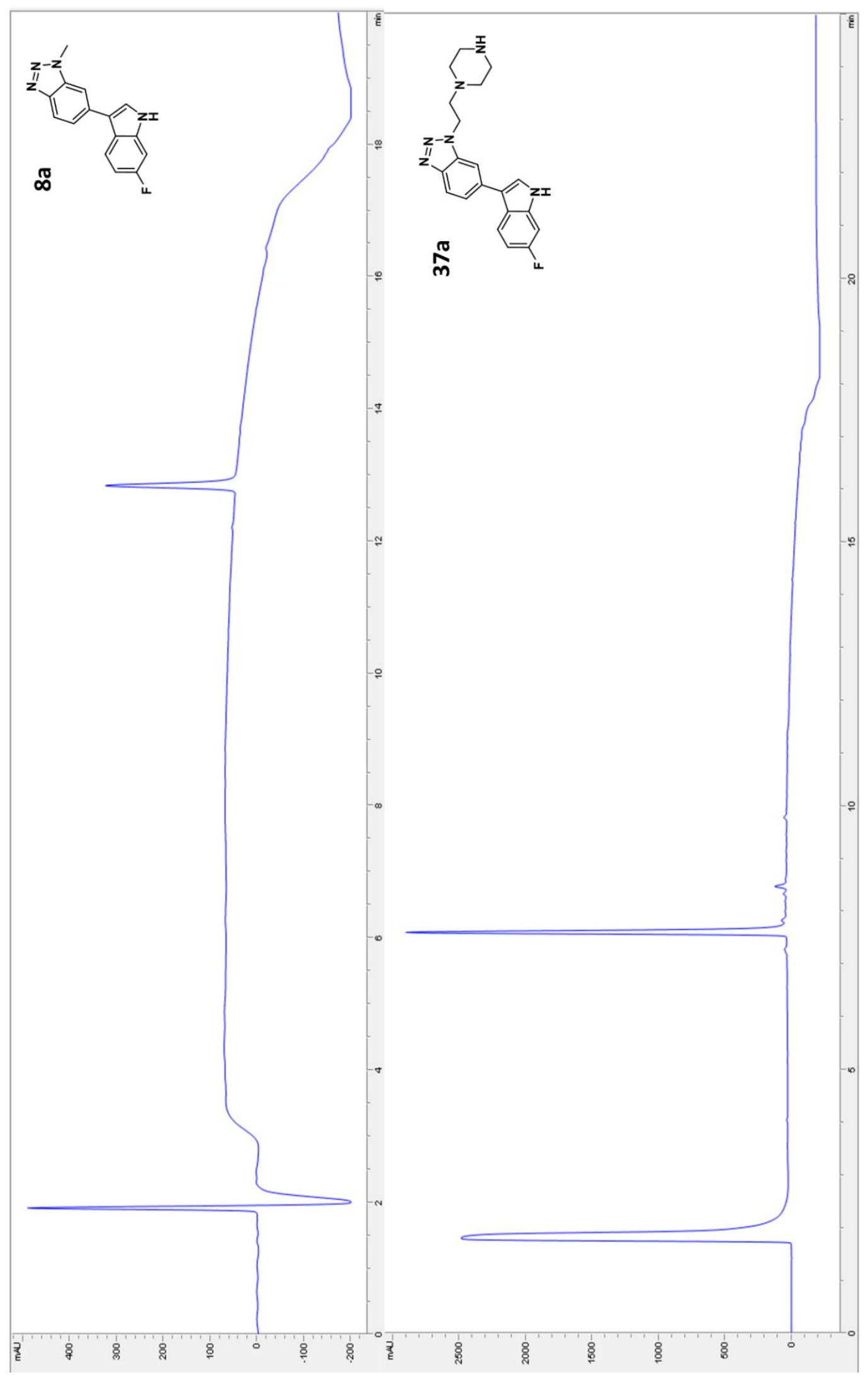




\section{Chemicals and Reagents}

General. All chemical reagents and solvents were used as obtained from various commercial sources (Merck/Sigma, Fluorochem, TCl, VWR) and were all used without further purification. Unless stated otherwise, reactions were performed under atmospheric pressure. Thin-layer chromatography (TLC) was performed using silica gel 60 F254 plates, with observation under UV at 254 and $366 \mathrm{~nm}$ Camag Lamp. For purification, flash-column chromatography on silica gel was performed. The compound characterization was done using ${ }^{1} \mathrm{H}$ and ${ }^{13} \mathrm{C}$ NMR; recorded on a $400 \mathrm{MHz}$ Bruker Ultrashield Advance II spectrometer, and their spectra were analyzed using the TopSpin 4 software. NMR analysis was performed in either $\mathrm{CDCl}_{3}$, DMSO- $d 6$ or $\mathrm{MeOD}$, all purchased from Eurisotop. Chemical shifts are in parts per million (ppm), coupling constants $(\mathrm{J})$ are in hertz $(\mathrm{Hz})$. Standard abbreviations indicating multiplicity were used as follows: $\mathrm{s}=$ singlet, $\mathrm{d}=$ doublet $\mathrm{t}=$ triplet $\mathrm{dd}=$ double doublet, $\mathrm{q}=$ quartet, $\mathrm{m}=$ multiplet, $\mathrm{br}=$ broad. Melting points $/$ ranges were determined using an electrothermal IA9000 apparatus and are reported uncorrected. High-resolution mass spectra were carried out on a Thermofisher Scientific LTQ-Orbitrap XL hybrid mass spectrometer (Thermo Fisher Scientific, Bremen, Germany). Data were acquired in full-scan MS positive ion mode. External calibration was performed before data acquisition. Tuning of the electrospray ion source was performed accordingly. The following electrospray inlet conditions were used: flow rate, $100 \mu \mathrm{L} \mathrm{min-1}$; spray voltage, $5 \mathrm{kV}$; sheath gas (N2) flow rate, 20 au; auxiliary gas (N2) flow rate, 10 au; capillary temperature, $275^{\circ} \mathrm{C}$; capillary voltage, $45 \mathrm{~V}$; tube lens, $80 \mathrm{~V}$.

$\log \mathrm{D}_{7.4}$ calculations were performed using Chemicalize (ChemAxon).

\section{Synthesis}

\section{Synthesis of boronic esters}

1.1. 1-(phenylsulfonyl)-3-(4,4,5,5-tetramethyl-1,3,2-dioxaborolan-2-yl)-1H-indole (41).

- 3-bromo-1H-indole (41a)

Indole (10 g, $85.36 \mathrm{mmol})$ was dissolved in $100 \mathrm{~mL}$ of pyridine. After what, pyridinium tribromide (1.25 eq, $34 \mathrm{~g}$ ) in $100 \mathrm{~mL}$ pyridine was added dropwise using a dropping funnel and the reaction was left to stir for 1 hour. Next, ice water was added and the reaction mixture was extracted with $\mathrm{Et}_{2} \mathrm{O}(3 \mathrm{x})$. The $\mathrm{OL}$ was washed with $6 \mathrm{~N} \mathrm{HCl}$ and saturated $\mathrm{NaHCO}_{3}$, dried with $\mathrm{MgSO}_{4}$, filtered and concentrated in 
vacuo to afford 12.7 grams of (41a) as a beige powder, which was used as such in the next step. Yield=

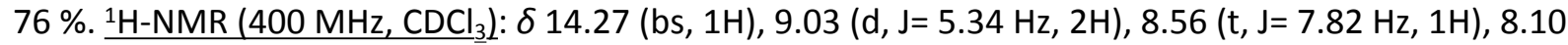
$(\mathrm{t}, \mathrm{J}=6.94 \mathrm{~Hz}, 2 \mathrm{H})$. El-MS (m/z): $337[\mathrm{M}]^{+}$

\section{- 3-bromo-1-(phenylsulfonyl)-1H-indole (41b)}

A 2-necked round bottom (RB) flask was charged with 3-bromo-1H-indole $(12.5 \mathrm{~g}, 63.77 \mathrm{mmol})$ and TBAHS $(3.25 \mathrm{~g}, 9.56 \mathrm{mmol})$ in $300 \mathrm{~mL}$ toluene at $0^{\circ} \mathrm{C}$. Next $300 \mathrm{~mL}$ of a $50 \%$ aqueous $\mathrm{NaOH}$ solution and benzenesulfonyl chloride $(16.9 \mathrm{~g}, 12.3 \mathrm{~mL}, 95.66 \mathrm{mmol})$ were added dropwise. The reaction mixture was left to stir at rt overnight. After TLC showed full conversion, the OL layer was separated, acidified with $1 \mathrm{~N} \mathrm{HCl}$, neutralized with $\mathrm{NaHCO}_{3}$, washed with brine, dried with $\mathrm{Na}_{2} \mathrm{SO}_{4}$ and concentrated in vacuo to be used as such. $\mathbf{4 1} \mathbf{b}$ was obtained as a beige powder in quantitative yield. The compound is poorly stable, it was stored at $-20^{\circ} \mathrm{C}$ in the dark and used $<24 \mathrm{~h}$ after synthesis. $\underline{{ }^{1} \mathrm{H}-}$ NMR $\left(400 \mathrm{MHz}, C D C l_{3}\right): \delta 7.35(1 \mathrm{H}, \mathrm{t}, \mathrm{J}=7.45 \mathrm{~Hz}), 7.41(1 \mathrm{H}, \mathrm{t}, \mathrm{J}=7.32 \mathrm{~Hz}), 7.48(2 \mathrm{H}, \mathrm{t}, \mathrm{J}=7.77 \mathrm{~Hz}), 7.53$ $(1 \mathrm{H}, \mathrm{d}, \mathrm{J}=7.82 \mathrm{~Hz}), 7.58(1 \mathrm{H}, \mathrm{t}, \mathrm{J}=7.46 \mathrm{~Hz}), 7.66(1 \mathrm{H}, \mathrm{s}), 7.92(2 \mathrm{H}, \mathrm{d}, \mathrm{J}=7.61 \mathrm{~Hz}), 8.03(1 \mathrm{H}, \mathrm{d}, \mathrm{J}=8.29 \mathrm{~Hz})$. ${ }^{13} \mathrm{C} \mathrm{NMR}\left(100 \mathrm{MHz}, \mathrm{CDCl}_{3}\right): \delta 136.52 ; 134.91 ; 133.55 ; 130.02 ; 129.07 ; 126.82 ; 126.15 ; 125.63 ; 124.47 ;$ 119.77; 113.45; 98.92. El-MS (m/z): 337 [M]

- 1-(phenylsulfonyl)-3-(4,4,5,5-tetramethyl-1,3,2-dioxaborolan-2-yl)-1H-indole (41)

A RB flask was charged with 3-bromo-1-(phenylsulfonyl)-1H-indole (41b) (10 g, $29.76 \mathrm{mmol})$, bis(pinacolato)diboron $(10.2 \mathrm{~g}, 40.18 \mathrm{mmol})$ and KOAc $(8.8 \mathrm{~g}, 89.3 \mathrm{mmol})$ in $100 \mathrm{~mL} \mathrm{DMF}$. The reaction mixture was degassed for $20 \mathrm{~min}$, after which $\mathrm{Pd}(\mathrm{dppf}) \mathrm{Cl}_{2}(659 \mathrm{mg}, 3 \mathrm{~mol} \%)$ was added. The reaction was stirred at $80^{\circ} \mathrm{C}$ overnight. After TLC showed full conversion, EtOAc was added and the mixture was filtered over a pad of celite. The OL was evaporated in vacuo. The crude product was purified using flash chromatography ( hexane/EtOAc: $4 / 1$ ) to obtain $7.7 \mathrm{~g}$ of the desired product (67\%). ${ }^{1 \mathrm{H}-\mathrm{NMR}(400}$ $\left.\mathrm{MHz}, \mathrm{CDCl}_{3}\right): \delta 1.26(12 \mathrm{H}, \mathrm{s}), 7.30(2 \mathrm{H}, \mathrm{t}, \mathrm{J}=7.38 \mathrm{~Hz}), 7.36(1 \mathrm{H}, \mathrm{d}, \mathrm{J}=7.96 \mathrm{~Hz}), 7.42(3 \mathrm{H}, \mathrm{t}, \mathrm{J}=7.85 \mathrm{~Hz}), 7.46$ $-7.54(3 \mathrm{H}, \mathrm{m}, \mathrm{J}=7.27 \mathrm{~Hz}), 7.63(1 \mathrm{H}, \mathrm{s}), 7.88(2 \mathrm{H}, \mathrm{d}, \mathrm{J}=7.73 \mathrm{~Hz}), 8.00(1 \mathrm{H}, \mathrm{d}, \mathrm{J}=8.31 \mathrm{~Hz}) .{ }^{13 \mathrm{C}-N M R}(100$ $\left.\mathrm{MHz}, \mathrm{CDCl}_{3}\right): \delta 25.04 ; 83.52 ; 99.84 ; 113.56 ; 120.11 ; 124.01 ; 124.72 ; 125.88 ; 126.85 ; 129.44 ; 134.18$; 137.74.EI-MS (m/z): $383[\mathrm{M}]^{+}$

\section{2. tert-butyl 6-fluoro-3-(4,4,5,5-tetramethyl-1,3,2-dioxaborolan-2-yl)-1H-indole-1-} carboxylate (42)

- tert-butyl 6-fluoro-1H-indole-1-carboxylate (42a)

To a $0.4 \mathrm{M}$ solution of 6 -fluoro- $1 \mathrm{H}$-indole $(4 \mathrm{~g}, 29.62 \mathrm{mmol}$, 1equi) in DCM at r.t. were added di-tertbutyl dicarbonate $\left(8.4 \mathrm{~g}, 38.5 \mathrm{mmol}, 1.3\right.$ equi), $\mathrm{Et}_{3} \mathrm{~N}(0.60 \mathrm{mg}, 5.92 \mathrm{mmol}, 0.2$ equi) and $4-$ dimethylpyrridine $(0.72 \mathrm{~g}, 5.92 \mathrm{mmol}, 0.2$ equi). The solution was stirred under atm. $\mathrm{P}$ for $3 \mathrm{~h}$, then washed with $\mathrm{H}_{2} \mathrm{O}$ and Brine, dried with $\mathrm{MgSO}_{4}$ and concentrated in-vacuo. The product 42a was obtained as a orange/brown oil and was used without further purification. Quantitative yield. ${ }^{1} \mathrm{H} \mathrm{NMR}$ $\left(400 \mathrm{MHz}, \mathrm{CDCl}_{3}\right)$ : 国 $1.70(9 \mathrm{H}, \mathrm{s}), 6.55(1 \mathrm{H}, \mathrm{d}, \mathrm{J}=3,35 \mathrm{~Hz}), 7.00(1 \mathrm{H}, \mathrm{td}, \mathrm{J}=8.92 \mathrm{~Hz}, \mathrm{~J}=2.37 \mathrm{~Hz}), 7.49(1 \mathrm{H}, \mathrm{dd}$, $\mathrm{J}=8.98 \mathrm{~Hz}, \mathrm{~J}=5.45 \mathrm{~Hz}), 7.59(1 \mathrm{H}, \mathrm{d}, \mathrm{J}=3.12 \mathrm{~Hz}), 7.90(1 \mathrm{H}, \mathrm{d}, \mathrm{J}=8.5 \mathrm{OHz}) .{ }^{13} \mathrm{C} \mathrm{NMR}\left(100 \mathrm{MHz}, \mathrm{CDCl}_{3}\right)$ : 国 28.16 ; $84.09 ; 102.55$ (d, J=28.18 Hz) ; $107.02 ; 110.91$ (d, J=24,49 Hz); 121.46 (d, J=10.04 Hz); 126.19 ; $126.77 ; 135.35$ (d, J=12.83 Hz) ; $149.54 ; 160.85$ (d, J=238.88 Hz).

\section{- tert-butyl 3-bromo-6-fluoro-1H-indole-1-carboxylate (42b)}

To a $0.1 \mathrm{M}$ solution of $42 \mathrm{a}(6,97 \mathrm{~g}, 29,62 \mathrm{mmol}, 1 \mathrm{eq})$ in DCM was added $\mathrm{N}$-bromosuccinimide (NBS) $(6.85 \mathrm{~g}, 38.50 \mathrm{mmol}, 1.1$ equi $)$. The solution was stirred at atm. P. under reflux for $6 \mathrm{~h} .0 .1$ equi of NBS were added after $2 \mathrm{~h}, 4 \mathrm{~h}$, and $5 \mathrm{~h}$ (If added all at once, the reaction led to the formation of an undesired dibrominated compound). The reaction mixture was subsequently washed with $\mathrm{H}_{2} \mathrm{O}, 1 \mathrm{M} \mathrm{NaOH}$ and Brine, dried with $\mathrm{MgSO}_{4}$ and concentrated in-vacuo. The obtained residue was crystallized in EtOH. Pink crystals. Yield: $80 \%$. ${ }^{1} \mathrm{H} \mathrm{NMR}\left(400 \mathrm{MHz}, \mathrm{CDCl}_{3}\right)$ : $1.69(9 \mathrm{H}, \mathrm{s}), 7.08(1 \mathrm{H}, \mathrm{td}, \mathrm{J}=8.81 \mathrm{~Hz}, \mathrm{~J}=1.9 \mathrm{~Hz}), 7.47$ 
$(1 \mathrm{H}, \mathrm{dd}, \mathrm{J}=8.56 \mathrm{~Hz}, \mathrm{~J}=5.3 \mathrm{~Hz}), 7.63(1 \mathrm{H}, \mathrm{s}), 7.91(1 \mathrm{H}, \mathrm{s}) .{ }^{13} \mathrm{C} \mathrm{NMR}\left(100 \mathrm{MHz} \mathrm{CDCl}_{3}\right)$ : $28.11 ; 84.77 ; 97.59$; 102.84 (d, J=29.07 Hz) ; 111.69 (d, J=24.70 Hz) ; 120.43 (d, J=10.03 Hz) ; $125.04 ; 125.74 ; 134.72$ (d, $\mathrm{J}=11.67 \mathrm{~Hz}) ; 148.63 ; 161.57$ (d, J=241.79 Hz).

- tert-butyl 6-fluoro-3-(4,4,5,5-tetramethyl-1,3,2-dioxaborolan-2-yl)-1H-indole-1carboxylate (42)

In a flame-dried RB flask under $\mathrm{N}_{2}$, tert-butyl 3-bromo-6-fluoro-1H-indole-1-carboxylate (42b) $(6 \mathrm{~g}$, $19,09 \mathrm{mmol}, 1 \mathrm{eq})$ was dissolved in dry THF $(70 \mathrm{~mL})$. The solution was stirred at $-78^{\circ} \mathrm{C}$ several minutes before dropwise addition of $\mathrm{n}$-BuLi $(27,5 \mathrm{~mL}$ of a $2.5 \mathrm{M}$ solution in hexanes, $68,75 \mathrm{mmol}, 3,6 \mathrm{eq})$. After $30 \mathrm{~min}$., a solution of bispinacolatodiboron $(9,7 \mathrm{~g}, 38,19 \mathrm{mmol}, 2 \mathrm{eq})$ in $24 \mathrm{~mL}$ of dry THF was added to the reaction mixture. After $3 \mathrm{~h}$, the reaction was quenched with saturated $\mathrm{NH}_{4} \mathrm{Cl}_{\mathrm{aq}}$. The mixture was extracted 3x with EtOAc. The combined $\mathrm{OL}$ was washed with $\mathrm{H} 2 \mathrm{O}$ and Brine, dried with $\mathrm{Na}_{2} \mathrm{SO}_{4}$ and concentrated in-vacuo. The residue was purified by flash chromatography in Cyclohexane/EtOAc (9:1). Optionally, when purity was insufficient, a crystallisation in $\mathrm{MeOH}$ was performed. White powder. Yield : $43 \%$. ${ }^{1} \mathrm{H} \mathrm{NMR}\left(400 \mathrm{MHz} \mathrm{CDCl}_{3}\right)$ : 回 $1.39(12 \mathrm{H}, \mathrm{s}), 1.67(9 \mathrm{H}, \mathrm{s}), 7.03(1 \mathrm{H}, \mathrm{td}, \mathrm{J}=9.00 \mathrm{~Hz}, \mathrm{~J}=2.30 \mathrm{~Hz})$, 7.89 - $7.92(2 \mathrm{H}, \mathrm{m}), 7.99(1 \mathrm{H}, \mathrm{s}) .{ }^{13} \mathrm{C} \mathrm{NMR}\left(100 \mathrm{MHz}_{2} \mathrm{CDCl}_{3}\right)$ : $24.90 ; 28.16 ; 83.47 ; 84.29 ; 102.26(\mathrm{~d}$, $\mathrm{J}=28.59 \mathrm{~Hz}) ; 111.08$ (d, J=23.83Hz); 123.23 (d, J=9.81Hz); $129.62 ; 135.53$ (d, J=3.19 Hz); 136.26 (d, $\mathrm{J}=12.79 \mathrm{~Hz}) ; 149.17 ; 160.85(\mathrm{~d}, \mathrm{~J}=239.66 \mathrm{~Hz})$.

1.3. Synthesis of 1-(Phenylsulfonyl)-3-indolylboronic acid MIDA ester (4-methyl-8-(1(phenylsulfonyl)-1H-indol-3-yl)dihydro-4/4,8/4-[1,3,2]oxazaborolo[2,3b] $[1,3,2]$ oxazaborole-2,6(3H,5H)-dione) (43)

The synthesis was adapted from J. Am. Chem. Soc. 2009, 131, 20, 6961-6963. To a round-bottom flask equipped with a stir bar was added 1-(phenylsulfonyl)-3-indoleboronic acid ( 1 equi, $1 \mathrm{~g}, 3.32 \mathrm{mmol}$, purchased from Fluorochem), $\mathrm{N}$-methyliminodiacetic acid ( 1.5 equi, $0.732 \mathrm{~g}, 4.96 \mathrm{mmol}$ ), toluene (21 $\mathrm{mL}$ ) and DMSO $(11 \mathrm{~mL})$. The flask was fitted with a Dean-Stark trap and the Dean-Stark trap was fitted with a reflux condenser vented to ambient atmosphere. The stirred mixture was heated to reflux for $1 \mathrm{~h} 30$, after what the reaction was complete. Toluene was evaporated in vacuo. The product was precipitated with cyclohexane and filtered over a glass filter funnel. The final compound was obtained as a white powder with $85 \%$ yield. ${ }^{1} \mathrm{H} N M R\left(400 \mathrm{MHz}^{\mathrm{CDCl}} \mathrm{CDC}_{3}\right)$ : $2.56(3 \mathrm{H}, \mathrm{s}), 4.19(2 \mathrm{H}, \mathrm{d}, \mathrm{J}=17.21 \mathrm{~Hz})$, $4.39(2 \mathrm{H}, \mathrm{d}, \mathrm{J}=17.25 \mathrm{~Hz}), 7.22-7.29(1 \mathrm{H}, \mathrm{d}, \mathrm{J}=26.01 \mathrm{~Hz}), 7.32(1 \mathrm{H}, \mathrm{t}, \mathrm{J}=7.62 \mathrm{~Hz}), 7.52-7.63(3 \mathrm{H}, \mathrm{m})$, $7.69(1 \mathrm{H}, \mathrm{t}, \mathrm{J}=7.30 \mathrm{~Hz}), 7.74(1 \mathrm{H}, \mathrm{s}), 7.90(1 \mathrm{H}, \mathrm{d}, \mathrm{J}=8.16 \mathrm{~Hz}), 8.03(2 \mathrm{H}, \mathrm{d}, \mathrm{J}=7.80 \mathrm{~Hz}) .{ }^{13} \mathrm{C} \mathrm{NMR}(100 \mathrm{MHz}$, $\mathrm{CDCl}_{3}$ : 137.40 .

\section{Synthesis of $\mathbf{N}$-substituted benzotriazoles}

2.1. Bromo-1-(4-methoxybenzyl)-1H-benzo[d][1,2,3]triazole (44)

- 6-bromo-1H-benzo[d][1,2,3]triazole (44a)

4-bromobenzene-1,2-diamine ( $1 \mathrm{~g}, 5.35 \mathrm{mmol})$ was dissolved in $10 \mathrm{~mL}$ of glacial acetic acid, to this solution were added 2.5 equivalents of $\mathrm{NaNO}_{2}(0.92 \mathrm{~g}, 13.35 \mathrm{mmol})$ and the resulting mixture was sonicated for 20 minutes. The reaction mixture is poured onto $\mathrm{H}_{2} \mathrm{O}$ and the off-white precipitate was filtered and washed with $\mathrm{H}_{2} \mathrm{O}$ until neutrality. Yield: $86 \%$. ${ }^{1} \mathrm{H} \mathrm{NMR}\left(400 \mathrm{MHz}, \mathrm{CDCl}_{3}\right)$ : 圆 $7.57(1 \mathrm{H}, \mathrm{dd}$, $\mathrm{J}=1.29,8.76 \mathrm{~Hz}), 7.91(1 \mathrm{H}, \mathrm{d}, \mathrm{J}=8.75 \mathrm{~Hz}), 8.21(1 \mathrm{H}, \mathrm{s})$.

- 6-bromo-1-(4-methoxybenzyl)-1H-benzo[d][1,2,3]triazole + + 5-bromo-1-(4methoxybenzyl)-1H-benzo[d][1,2,3]triazole (44) 
Compound 8 (396mg,2 mmol), was dissolved in $20 \mathrm{~mL}$ DMF. To this solution were added 4methoxybenzyl chloride ( $0.6 \mathrm{~mL}, 2.2$ equi), TBAB ( $130 \mathrm{mg}, 0.2$ equi) and $\mathrm{NaH} 60 \%$ (168 mg, 2.1 equi). Agitated overnight at atm. P. and r.t. After completion, saturated $\mathrm{NH}_{4} \mathrm{Cl}_{\mathrm{aq}}$ was added and the mixture was extracted with EtOAc. The combined OL was washed with $\mathrm{CuSO}_{4(\mathrm{aq})}$ and Brine, dried with $\mathrm{Na}_{2} \mathrm{SO}_{4}$ and concentrated in vacuo. The residue was purified by flash chromatography (Cyclohexane/EtOAc 4:1). The product is obtained as a mix of two regioisomers that co-elute. ${ }^{1} \mathrm{H} \mathrm{NMR}\left(400 \mathrm{MHz}, \mathrm{CDCl}_{3}\right)$ : [ ] $3.79(6 \mathrm{H}, \mathrm{d}, \mathrm{J}=3.90 \mathrm{~Hz}), 5.75(4 \mathrm{H}, \mathrm{d}, \mathrm{J}=11.92 \mathrm{~Hz}), 6.87(4 \mathrm{H}, \mathrm{dd}, \mathrm{J}=6.44,8.41 \mathrm{~Hz}), 7.20-7.26(\mathrm{~m}), 7.44$ $(1 \mathrm{H}, \mathrm{dd}, \mathrm{J}=1.45,8.80 \mathrm{~Hz}), 7.47(1 \mathrm{H}, \mathrm{dd}, \mathrm{J}=1.51,8.78 \mathrm{~Hz}), 7.54(1 \mathrm{H}, \mathrm{s}), 7.92(1 \mathrm{H}, \mathrm{d}, \mathrm{J}=8.78 \mathrm{~Hz}), 8.21(1 \mathrm{H}$, $\mathrm{d}, \mathrm{J}=0.86 \mathrm{~Hz})$.

\subsection{Synthesis of $\mathbf{N}$-methylated 6-chloro-1H-benzo[d][1,2,3]triazoles (7b, $\mathbf{8 b}$, 9b)}

The procedure previously described by A. Carta et al. in Heterocycles, 65(10), 2471-2481; 2005

The commercially available 5-Chlorobenzotriazole (CAS = 94-97-3, $10 \mathrm{mmol}$ ) was dissolved in $25 \mathrm{~mL}$ of $\mathrm{NaOH}_{\text {aq }} 2 \mathrm{M}$. 1.6 equi. of $\mathrm{Me}_{2} \mathrm{SO}_{4}(16 \mathrm{mmol})$ were added. The reaction mixture was agitated at room temperature for $1 \mathrm{~h} 30$. The product gradually appeared in the form of an off-white precipitate and contained $3 \mathrm{~N}$-methylated regioisomers. A flash chromatography was performed using cyclohexane/EtOAc $(4: 1)$ as eluent. The structures were confirmed using diffraction X-Ray crystallography. Compounds eluted in the following order:

- 5-chloro-2-methyl-2H-benzo[d][1,2,3]triazole (7b)<smiles>Cn1nc2ccc(Cl)cc2n1</smiles>

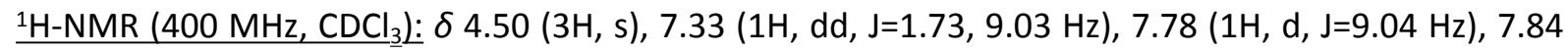
$(1 \mathrm{H}, \mathrm{d}, \mathrm{J}=1.11 \mathrm{~Hz}) .{ }^{13} \mathrm{C}-\mathrm{NMR}\left(100 \mathrm{MHz} \mathrm{CDCl}_{3}\right): \delta 43.37 ; 116.96 ; 119.06 ; 127.82 ; 132.13 ; 143.01$; 144.87 .

White powder. Yield $=23 \%$. Melting point $=57^{\circ} \mathrm{C}$

- 6-chloro-1-methyl-1H-benzo[d][1,2,3]triazole (8b)<smiles>Cn1nnc2ccc(Cl)cc21</smiles>

${ }^{1} \mathrm{H}-\mathrm{NMR}\left(400 \mathrm{MHz}, \mathrm{CDCl}_{3}\right) \delta 4.28(3 \mathrm{H}, \mathrm{s}), 7.33(1 \mathrm{H}, \mathrm{dd}, \mathrm{J}=1.58,8.83 \mathrm{~Hz}), 7.53(1 \mathrm{H}, \mathrm{d}, \mathrm{J}=0.99 \mathrm{~Hz}), 7.97$ $(1 \mathrm{H}, \mathrm{d}, \mathrm{J}=8.83 \mathrm{~Hz}) .{ }^{13} \mathrm{C}-\mathrm{NMR}\left(100 \mathrm{MHz}, \mathrm{CDCl}_{3}\right)$ 回 34,$36 ; 112,20 ; 121,14 ; 121,65 ; 127,61 ; 134,51$; 144,78 .

White powder. Yield $=31 \%$. Melting point $=120^{\circ} \mathrm{C}$

- 5-chloro-1-methyl-1H-benzo[d][1,2,3]triazole (9b)<smiles>Cn1[nH]c2cc(Cl)ccc21</smiles>

${ }^{1} \mathrm{H}-\mathrm{NMR}\left(400 \mathrm{MHz}, \mathrm{CDCl}_{3}\right): \delta 4.30(3 \mathrm{H}, \mathrm{s}), 7.46(2 \mathrm{H}, \mathrm{d}, \mathrm{J}=1.18 \mathrm{~Hz}), 8.01-8.05(1 \mathrm{H}, \mathrm{m})$.

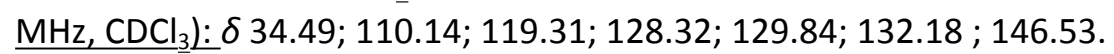


White powder.Yield $=27 \%$. Melting point $=85^{\circ} \mathrm{C}$

2.3. 2-(5-chloro-1H-benzo[d][1,2,3]triazol-1-yl)acetonitrile \& 2-(6-chloro-1Hbenzo[d][1,2,3]triazol-1-yl)acetonitrile (21b, 22b)

The commercially available 5-Chlorobenzotriazole (CAS $=94-97-3,20 \mathrm{mmol}$ ) was dissolved in anhydrous DMF (30 mL). Triethylamine $(22.86 \mathrm{mmol}, 3.81 \mathrm{~mL})$ and chloroacetonitrile $(31.26 \mathrm{mmol}$, $2 \mathrm{~mL}$ ) were added and the reaction was refluxed overnight (16h). The solution was basified to remove unreacted 5-Chlorobenzotriazole and the desired product was extracted using EtOAc. A column was performed using a gradient of Cyclohexane/EtOAc $(1: 0 \rightarrow 3: 1)$ as both products have very similar $R f$ values. The compounds eluted in the following order: $\mathbf{2 1} \mathbf{b}$ then $\mathbf{2 2} \mathbf{b}$. However, a significant number of fractions contained both products. Each product was crystallized in the same conditions: Cyclohexane was added to the obtained powder and heated until boiling. EtOAc was gradually added until complete dissolution.

- While conversion was total, reported yields are low due to purification process. Structures were confirmed with NOESY-NMR and X-ray diffraction crystallography.

- 2-(6-chloro-1H-benzo[d][1,2,3]triazol-1-yl)acetonitrile (21b)

Crystals were obtained as white long needles. Yield $=15 \%$. ${ }^{1} \mathrm{H}-\mathrm{NMR}\left(400 \mathrm{MHz}, \mathrm{CDCl}_{3}\right) \delta 8.06(\mathrm{~d}, \mathrm{~J}=8.84$ $\mathrm{Hz}, 1 \mathrm{H}), 7.70(\mathrm{~d}, \mathrm{~J}=0.83 \mathrm{~Hz}, 1 \mathrm{H}), 7.45(\mathrm{dd}, \mathrm{J}=8.84,3.44 \mathrm{~Hz}, 1 \mathrm{H}), 5.59(\mathrm{~s}, 2 \mathrm{H}) .{ }^{13} \mathrm{C}-\mathrm{NMR}\left(100 \mathrm{MHz}, \mathrm{CDCl}{ }_{3}\right)$ $\delta 35.77 ; 108.69 ; 112.07 ; 121.69 ; 126.36 ; 132.85 ; 135.71 ; 144.84$.

- 2-(5-chloro-1H-benzo[d][1,2,3]triazol-1-yl)acetonitrile (22b)

Crystals were obtained as white flakes. Yield $=10 \%$. ${ }^{1 \mathrm{H}-\mathrm{NMR}(400 \mathrm{MHz}, \mathrm{DMSO})} \delta 8.31(\mathrm{~d}, \mathrm{~J}=0.82 \mathrm{~Hz}$, $1 \mathrm{H}), 8.04\left(\mathrm{~d}, \mathrm{~J}=8.85 \mathrm{~Hz}, 1 \mathrm{H}\right.$ ), 7.74 (dd, J=8.82, 3.43 Hz, 1H), $6.20(\mathrm{~s}, 2 \mathrm{H}) .{ }^{13} \mathrm{C}-\mathrm{NMR}(100 \mathrm{MHz}, \mathrm{DMSO}) \delta$ $36.42 ; 112.59 ; 115.37 ; 119.37 ; 129.49 ; 129.88 ; 131.89 ; 146.22$.

\subsection{Substituted benzotriazoles}

2.4.1. List of $\mathrm{N}$-substituted benzotriazoles obtained starting from 4-Bromo-1-fluoro-2nitrobenzene [CAS 364-73-8]:

- 5-bromo-1-(2,2,2-trifluoroethyl)-1H-benzo[d][1,2,3]triazole (18d)<smiles>FC(F)(F)Cn1nnc2cc(Br)ccc21</smiles>

- 4-bromo-2-nitro-N-(2,2,2-trifluoroethyl)aniline (18b): 4-Bromo-1-fluoro-2-nitrobenzene $(500 \mathrm{mg}$, $2.27 \mathrm{mmol}$ ) and trifluoroethanolamine. $\mathrm{HCl}$ were dissolved in anhydrous THF $(4 \mathrm{~mL})$ and DIEA $(1.16 \mathrm{~mL}$, $6.82 \mathrm{mmol}$ ) under $\mathrm{N}_{2}$. The resulting mixture was refluxed for $16 \mathrm{~h}$. After cooling to room temperature, the solvent was removed in-vacuo. Then, the residue was resuspended in $10 \mathrm{~mL}$ water and extracted 3 times with EtOAc. The $\mathrm{OL}$ was dried with $\mathrm{Na}_{2} \mathrm{SO}_{4}$ and evaporated to yield $620 \mathrm{mg}(2.07 \mathrm{mmol})$ of a bright yellow powder containing 19b. Yield $=91 \%$ Used without further purification in the next step. ${ }^{1} \mathrm{H}-\mathrm{NMR}\left(400 \mathrm{MHz}_{\mathrm{CDCl}}\right)$ : $\delta 3.98(2 \mathrm{H}, \mathrm{qn}, \mathrm{J}=8.12 \mathrm{~Hz}), 6.88(1 \mathrm{H}, \mathrm{d}, \mathrm{J}=9.12 \mathrm{~Hz}), 7.59(1 \mathrm{H}, \mathrm{dd}, \mathrm{J}=2.09,9.11$ $\mathrm{Hz}), 8.21(1 \mathrm{H}, \mathrm{dd}, \mathrm{J}=2.35,6.51 \mathrm{~Hz}), 8.36(1 \mathrm{H}, \mathrm{d}, \mathrm{J}=2.23 \mathrm{~Hz})$. 
- 4-bromo- $N^{1}-(2,2,2$-trifluoroethyl)benzene-1,2-diamine (18c). Obtained following the general reduction procedure. After evaporation, the crude extract was immediately engaged in the next step.

- 5-bromo-1-(2,2,2-trifluoroethyl)-1H-benzo[d][1,2,3]triazole (18d) was obtained using the general procedure. Flash chromatography purification: Cyclohexane/EtOAc (9:1). Beige powder. Yield over two steps: $60 \% .5 .27-5.15(\mathrm{~m}, 2 \mathrm{H}), 7.56(\mathrm{~d}, \mathrm{~J}=8.72 \mathrm{~Hz}, 1 \mathrm{H}), 7.78(\mathrm{~s}, 1 \mathrm{H}), 7.99(\mathrm{~d}, \mathrm{~J}=8.76 \mathrm{~Hz}, 1 \mathrm{H}) .{ }^{13} \mathrm{C}-\mathrm{NMR}$ $\left(100 \mathrm{MHz}, \mathrm{CDCl}_{3}\right) \delta 49.23 \& 49.60\left(-\mathrm{CF}_{3}\right) ; 110.40 ; 117.96 ; 123.13 ; 132.12 ; 132.24 ; 147.26$. Yield: $56 \%$.

- 5-bromo-1-(cyclopropylmethyl)-1H-benzo[d][1,2,3]triazole (20d)<smiles>Brc1ccc2c(c1)nnn2CC1CC1</smiles>

- 4-bromo-N-(cyclopropylmethyl)-2-nitroaniline (20b) was synthesized according to the general procedure using cyclopropylmethanamine. Quantitative yield. Obtained as a bright orange oil. ${ }^{1} \mathrm{H}-\mathrm{NMR}$ $\left(400 \mathrm{MHz}, \mathrm{CDCl}_{3}\right): \delta 0.35-0.30(\mathrm{~m}, 2 \mathrm{H}), 0.69-0.63(\mathrm{~m}, 2 \mathrm{H}), 1.21-1.14(\mathrm{~m}, 1 \mathrm{H}), 3.14(\mathrm{dd}, \mathrm{J}=6.37,5.41$ $\mathrm{Hz}), 6.73(\mathrm{~d}, \mathrm{~J}=9.16 \mathrm{~Hz}, 1 \mathrm{H}), 7.48(\mathrm{~d}, \mathrm{~J}=9.16 \mathrm{~Hz}, 1 \mathrm{H}), 8.11$ (bs, $1 \mathrm{H}), 8.32(\mathrm{~s}, 1 \mathrm{H}) .{ }^{13} \mathrm{C}-\mathrm{NMR}(100 \mathrm{MHz}$, $\left.\mathrm{CDCl}_{3}\right): 48.18 ; 106.24 ; 115.60 ; 128.94 ; 138.90$.

- 4-bromo-N1-(cyclopropylmethyl)benzene-1,2-diamine (20c). Obtained by reducing $20 \mathrm{~b}$ using the general procedure, reaction time $=1 \mathrm{~h}$. The crude extract was immediately used in the following step.

- 5-bromo-1-(cyclopropylmethyl)-1H-benzo[d][1,2,3]triazole (20d) was obtained by using the general cyclisation procedure. Reaction time $=30 \mathrm{~min}$. The compound was purified using flash chromatography (cyclohexane/EtOAc 3:1) and obtained as a dark red oil that solidified overnight. Yield over two steps

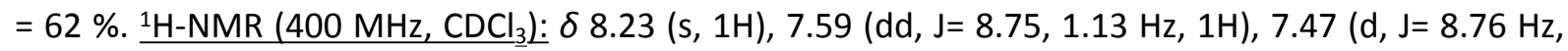
$1 \mathrm{H}), 4.51(\mathrm{~d}, \mathrm{~J}=7.11 \mathrm{~Hz}, 2 \mathrm{H}), 1.42-1.35(\mathrm{~m}, 1 \mathrm{H}), 0.70-0.66(\mathrm{~m}, 2 \mathrm{H}), 0.52-0.47(\mathrm{~m}, 2 \mathrm{H}) .{ }^{13} \mathrm{C}-\mathrm{NMR}(100$

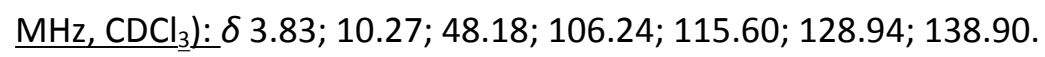

- 2-(5-bromo-1H-benzo[d][1,2,3]triazol-1-yl)-N,N-dimethylethan-1-amine (26d)<smiles>CN(C)CCn1nnc2cc(Br)ccc21</smiles>

$N^{1}$-(4-bromo-2-nitrophenyl)- $N^{2}, N^{2}$-dimethylethane-1,2-diamine (26b) was synthesized according to the general procedure using $N^{1}, N^{1}$-dimethylethane-1,2-diamine. Yield: $84 \%$. Orange powder. ${ }^{1} \mathrm{H} N M R$ $\left(400 \mathrm{MHz}, \mathrm{CDCl}_{3}\right)$ : 国 $2.30(6 \mathrm{H}, \mathrm{s}), 2.63(2 \mathrm{H}, \mathrm{t}, \mathrm{J}=6.18 \mathrm{~Hz}), 3.32(2 \mathrm{H}, \mathrm{dd}, \mathrm{J}=5.92,11.05 \mathrm{~Hz}), 6.74(1 \mathrm{H}, \mathrm{d}$, $\mathrm{J}=9.16 \mathrm{~Hz}), 7.48(1 \mathrm{H}, \mathrm{dd}, \mathrm{J}=2.09,9.13 \mathrm{~Hz}), 8.28-8.38(2 \mathrm{H}, \mathrm{m}) .{ }^{13} \mathrm{C} \mathrm{NMR}\left(100 \mathrm{MHz}, \mathrm{CDCl}_{3}\right):$ ? 40.77; $45.26 ; 57.20 ; 106.08 ; 115.71 ; 128.92 ; 132.21 ; 138.80 ; 144.27$.

4-bromo-N1-(2-(dimethylamino)ethyl)benzene-1,2-diamine (26c) Obtained by reducing 26b using the general procedure. The crude extract was immediately used in the following step.

2-(5-bromo-1H-benzo[d][1,2,3]triazol-1-yl)-N,N-dimethylethan-1-amine (26d). Flash chromatography (cyclohexane/EtOAc 3:1 $\rightarrow$ 0:1). Yield over two steps: $65 \%$. Dark brown powder. ${ }^{1} \mathrm{H}$ NMR $(400 \mathrm{MHz}$, $\left.\mathrm{CDCl}_{3}\right)$ : $2.30(6 \mathrm{H}, \mathrm{s}), 2.88(2 \mathrm{H}, \mathrm{t}, \mathrm{J}=6.65 \mathrm{~Hz}), 4.71(2 \mathrm{H}, \mathrm{t}, \mathrm{J}=6.65 \mathrm{~Hz}), 7.47(1 \mathrm{H}, \mathrm{d}, \mathrm{J}=8.75 \mathrm{~Hz}), 7.57(1 \mathrm{H}$, 
dd, J=1.22, $8.76 \mathrm{~Hz}), 8.20(1 \mathrm{H}, \mathrm{s}) .{ }^{13} \mathrm{C} \mathrm{NMR}\left(100 \mathrm{MHz}, \mathrm{CDCl}_{3}\right)$ : 回 45.56; 46.84; 58.41; 110.92; 117.11; $122.56 ; 130.64 ; 132.25 ; 147.09$.

- 5-bromo-1-((tetrahydrofuran-3-yl)methyl)-1H-benzo[d][1,2,3]triazole (28)<smiles>Brc1ccc2c(c1)nnn2CC1CCOC1</smiles>

4-bromo-2-nitro-N-((tetrahydrofuran-3-yl)methyl)aniline (28b). Synthesized according to the general procedure using (tetrahydrofuran-3-y/)methanamine. Compound was obtained in quantitative yield as an orange powder. ${ }^{1} \mathrm{H}-\mathrm{NMR}\left(400 \mathrm{MHz}, \mathrm{CDCl}_{3}\right): \delta 1.65-1.81(1 \mathrm{H}, \mathrm{m}), 2.14-2.25(1 \mathrm{H}, \mathrm{m}), 2.61-2.73$ $(1 \mathrm{H}, \mathrm{m}), 3.22-3.36(2 \mathrm{H}, \mathrm{m}, \mathrm{J}=4.31 \mathrm{~Hz}), 3.67(1 \mathrm{H}, \mathrm{dd}, \mathrm{J}=4.83,8.89 \mathrm{~Hz}), 3.80(1 \mathrm{H}, \mathrm{dd}, \mathrm{J}=7.90,15.69 \mathrm{~Hz})$, $3.90(1 \mathrm{H}, \mathrm{dd}, \mathrm{J}=6.94,8.77 \mathrm{~Hz}), 3.94-4.02(1 \mathrm{H}, \mathrm{m}), 6.78(1 \mathrm{H}, \mathrm{dd}, \mathrm{J}=1.64,9.08 \mathrm{~Hz}), 7.02(1 \mathrm{H}, \mathrm{d}, \mathrm{J}=1.48$ $\mathrm{Hz}), 8.04(1 \mathrm{H}, \mathrm{d}, \mathrm{J}=9.08 \mathrm{~Hz}), 8.08-8.19(1 \mathrm{H}, \mathrm{m}) .{ }^{13} \mathrm{C}-\mathrm{NMR}\left(100 \mathrm{MHz}, \mathrm{CDCl}_{3}\right): \delta 26.91 ; 30.07 ; 38.47 ;$ $46.27 ; 67.72 ; 71.27 ; 116.31 ; 118.98 ; 128.21 ; 131.01 ; 131.83 ; 145.69$.

4-bromo- $N^{1}-(($ tetrahydrofuran-3-yl)methyl)benzene-1,2-diamine (28c). Obtained following the general reduction procedure. After evaporation, the crude extract was immediately engaged in the next step.

5-bromo-1-((tetrahydrofuran-3-yl)methyl)-1H-benzo[d][1,2,3]triazole (28d). Obtained following the general cyclisation procedure. The obtained compound was purified using flash chromatography

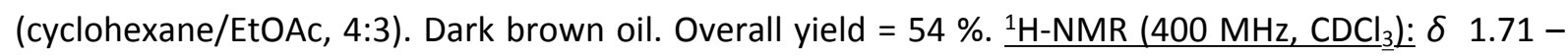
$1.82(1 \mathrm{H}, \mathrm{m}), 2.03-2.14(1 \mathrm{H}, \mathrm{m}), 2.96-3.08(1 \mathrm{H}, \mathrm{m}), 3.68(1 \mathrm{H}, \mathrm{dd}, \mathrm{J}=4.69,9.10 \mathrm{~Hz}), 3.76-3.85(2 \mathrm{H}$, m), $4.57(2 \mathrm{H}, \mathrm{d}, \mathrm{J}=7.74 \mathrm{~Hz}), 7.49(1 \mathrm{H}, \mathrm{dd}, \mathrm{J}=1.35,8.80 \mathrm{~Hz}), 7.74(1 \mathrm{H}, \mathrm{d}, \mathrm{J}=0.66 \mathrm{~Hz}), 7.95(1 \mathrm{H}, \mathrm{d}, \mathrm{J}=8.80$ $\mathrm{Hz})$.

- 5-bromo-1-((tetrahydrofuran-2-yl)methyl)-1H-benzo[d][1,2,3]triazole (30d)<smiles>Brc1ccc2c(c1)nnn2CC1CCCO1</smiles>

- 4-bromo-2-nitro-N-((tetrahydrofuran-2-yl)methyl)aniline (30b) Synthesized according to the general procedure using 2-(aminomethyl)tetrahydrofuran. The reaction mixture was agitated for $1 \mathrm{~h}$ at room temperature. After cooling to $0^{\circ} \mathrm{C}$, the precipitate was filtered and washed with cold EtOH. The product was obtained as a bright orange powder with an $82 \%$ yield. ${ }^{1} \mathrm{H}-\mathrm{NMR}\left(400 \mathrm{MHz}, \mathrm{CDCl}_{3}\right): \delta 1.65-1.79$ $(\mathrm{m}, 1 \mathrm{H}), 1.93-2.01(\mathrm{~m}, 2 \mathrm{H}), 2.05-2.13(\mathrm{~m}, 1 \mathrm{H}), 3.29-3.36(\mathrm{~m}, 1 \mathrm{H}), 3.42-3.46(\mathrm{~m}, 1 \mathrm{H}), 3.79-3.86(\mathrm{~m}$, $1 \mathrm{H}), 3.91-3.99(\mathrm{~m}, 1 \mathrm{H}), 4.15-4.22(\mathrm{~m}, 1 \mathrm{H}), 6.81(\mathrm{~d}, \mathrm{~J}=9.17 \mathrm{~Hz}, 1 \mathrm{H}), 7.48(\mathrm{~d}, \mathrm{~J}=9.12 \mathrm{~Hz}, 1 \mathrm{H}), 8.23$ (bs, $1 \mathrm{H}), 8.31(\mathrm{~s}, 1 \mathrm{H}) .{ }^{13} \mathrm{C}-\mathrm{NMR}\left(100 \mathrm{MHz}, \mathrm{CDCl}_{3}\right) \delta 25.86 ; 29.15 ; 30.95 ; 47.07 ; 68.57 ; 106.44 ; 115.71 ; 128.93$; $132.40 ; 138.84 ; 144.56$.

-4-bromo-N-((tetrahydrofuran-2-yl)methyl)benzene-1,2-diamine (30c). Obtained following the general reduction procedure. After evaporation, the crude extract was immediately engaged in the next step.

- 5-bromo-1-((tetrahydrofuran-2-yl)methyl)-1H-benzo[d][1,2,3]triazole (30d). Obtained following the general cyclisation procedure. The obtained compound was purified using flash chromatography (cyclohexane/EtOAc, 4:3). Dark red oil. Overall yield $37 \%$. ${ }^{1} \mathrm{H}-\mathrm{NMR}\left(400 \mathrm{MHz}, \mathrm{CDCl}_{3}\right): \delta 1.56-1.64(\mathrm{~m}$, $1 \mathrm{H}), 1.68-1.76(\mathrm{~m}, 1 \mathrm{H}), 1.79-1.89(\mathrm{~m}, 1 \mathrm{H}), 2.03-2.12(\mathrm{~m}, 1 \mathrm{H}), 3.64-3.73(\mathrm{~m}, 2 \mathrm{H}), 4.35-4.41(\mathrm{~m}, 1 \mathrm{H})$, 
$4.68(\mathrm{dd}, \mathrm{J}=14.49,5.39 \mathrm{~Hz}, 1 \mathrm{H}), 4.80$ (dd, J=14.49Hz, $3.48 \mathrm{~Hz}, 2 \mathrm{H}), 7.54-7.63(\mathrm{~m}, 2 \mathrm{H}), 8.19(\mathrm{~s}, 1 \mathrm{H}) .{ }^{13} \mathrm{C}-$

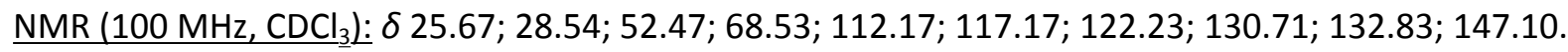

- 4-(2-(5-bromo-1H-benzo[d][1,2,3]triazol-1-yl)ethyl)morpholine (32d)<smiles>Brc1ccc2c(c1)nnn2CCN1CCOCC1</smiles>

-4-bromo-N-(2-morpholinoethyl)-2-nitroaniline (32b). Synthesized according to the general procedure using 4-(2-aminoethyl)morpholine. The precipitate was filtered and washed with cold EtOH. The

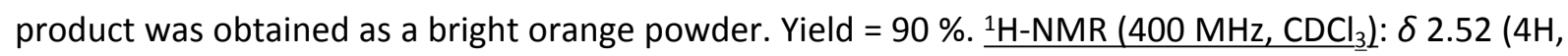
m), $2.72(2 \mathrm{H}, \mathrm{t}, \mathrm{J}=6.06 \mathrm{~Hz}), 3.34(2 \mathrm{H}, \mathrm{dd}, \mathrm{J}=10.83,5.63 \mathrm{~Hz}), 3.76(4 \mathrm{H}, \mathrm{t}, \mathrm{J}=4.39 \mathrm{~Hz}), 6.73(2 \mathrm{H}, \mathrm{d}, \mathrm{J}=9.13$ $\mathrm{Hz}), 7.49(2 \mathrm{H}, \mathrm{dd}, \mathrm{J}=1.70,9.08 \mathrm{~Hz}), 8.32(2 \mathrm{H}, \mathrm{d}, \mathrm{J}=1.94 \mathrm{~Hz}), 8.53(1 \mathrm{H}, \mathrm{s}) .{ }^{13} \mathrm{C}-\mathrm{NMR}\left(100 \mathrm{MHz}, \mathrm{CDCl}_{3}\right) \delta$ $39.40 ; 53.14 ; 55.87 ; 67.00 ; 106.20 ; 115.75 ; 128.99 ; 132.30 ; 138.85 ; 144.17$.

- 4-bromo-N-(2-morpholinoethyl)benzene-1,2-diamine (32c). Obtained following the general reduction procedure. Brown oil. Yield: $89 \%$. ${ }^{1} \mathrm{H}-\mathrm{NMR}\left(400 \mathrm{MHz}, \mathrm{CDCl}_{3}\right): \delta 2.43-2.53(4 \mathrm{H}, \mathrm{m}), 2.67(2 \mathrm{H}, \mathrm{t}, \mathrm{J}=5.76$ $\mathrm{Hz}), 3.13(2 \mathrm{H}, \mathrm{t}, \mathrm{J}=5.77 \mathrm{~Hz}), 3.47(2 \mathrm{H}, \mathrm{s}), 3.72(4 \mathrm{H}, \mathrm{t}, \mathrm{J}=4.33 \mathrm{~Hz}), 6.49(1 \mathrm{H}, \mathrm{d}, \mathrm{J}=8.35 \mathrm{~Hz}), 6.82(1 \mathrm{H}, \mathrm{d}$, $\mathrm{J}=1.59 \mathrm{~Hz}), 6.89(1 \mathrm{H}, \mathrm{q}, \mathrm{J}=3.32 \mathrm{~Hz})$.

- 4-(2-(5-bromo-1H-benzo[d][1,2,3]triazol-1-yl)ethyl)morpholine (32d). Obtained following the general cyclisation procedure. The crude was purified using flash chromatography cyclohexane/EtOAc $(1: 1 \rightarrow$ 2:1). The compound was obtained as a brown powder. Yield $=43 \% .{ }^{1} \mathrm{H}-\mathrm{NMR}\left(400 \mathrm{MHz}, \mathrm{CDCl}_{3}\right) \delta 2.46$ - $2.55(4 \mathrm{H}, \mathrm{m}) .2 .93(2 \mathrm{H}, \mathrm{t}, \mathrm{J}=6.43 \mathrm{~Hz}), 3.59-3.67(4 \mathrm{H}, \mathrm{m}), 4.74(2 \mathrm{H}, \mathrm{t}, \mathrm{J}=6.43 \mathrm{~Hz}), 7.47(1 \mathrm{H}, \mathrm{d}, \mathrm{J}=8.68$ $\mathrm{Hz}), 7.58(1 \mathrm{H}, \mathrm{d}, \mathrm{J}=8.53 \mathrm{~Hz}), 8.22(1 \mathrm{H}, \mathrm{s}) .{ }^{13} \mathrm{C}-\mathrm{NMR}\left(100 \mathrm{MHz}, \mathrm{CDCl}_{3}\right) \delta$ 46.13; 53.64; 57.63; 66.84; $110.89 ; 117.14 ; 122.65 ; 130.62 ; 132.29 ; 147.11$.

- tert-butyl 4-((5-bromo-1H-benzo[d][1,2,3]triazol-1-yl)methyl)piperidine-1-carboxylate (34d)<smiles>CC(C)(C)OC(=O)N1CCC(Cn2nnc3cc(Br)ccc32)CC1</smiles>

tert-butyl 4-(((4-bromo-2-nitrophenyl)amino)methyl)piperidine-1-carboxylate (34b). Synthesized according to the general procedure using tert-butyl 4-(aminomethyl)piperidine-1-carboxylate. An orange oil was obtained following evaporation under reduced pressure. Yield: $86 \%$. $\underline{{ }^{1} \mathrm{H}-\mathrm{NMR}(400 \mathrm{MHz}}$, $\left.\mathrm{CDCl}_{3}\right): \delta 1.36-1.49(11 \mathrm{H}, \mathrm{m}), 1.59-1.71(1 \mathrm{H}, \mathrm{m}), 1.78(2 \mathrm{H}, \mathrm{d}, \mathrm{J}=10.40 \mathrm{~Hz}), 2.83(2 \mathrm{H}, \mathrm{t}, \mathrm{J}=11.76 \mathrm{~Hz})$, $3.08(2 \mathrm{H}, \mathrm{t}, \mathrm{J}=5.82 \mathrm{~Hz}), 3.28(2 \mathrm{H}, \mathrm{d}, \mathrm{J}=11.92 \mathrm{~Hz}), 4.66-4.76(1 \mathrm{H}, \mathrm{m}), 7.07(1 \mathrm{H}, \mathrm{d}, \mathrm{J}=8.48 \mathrm{~Hz}), 7.22(1 \mathrm{H}$, s), $7.66(1 \mathrm{H}, \mathrm{d}, \mathrm{J}=8.64 \mathrm{~Hz})$.

tert-butyl 4-(((2-amino-4-bromophenyl)amino)methyl)piperidine-1-carboxylate (34c). Obtained following the general reduction procedure. Yield: $73 \%$. ${ }^{1} \mathrm{H}-\mathrm{NMR}\left(400 \mathrm{MHz}, \mathrm{CDCl}_{3}\right): \delta 1.32-1.42(2 \mathrm{H}$, m), $1.46(9 \mathrm{H}, \mathrm{d}, \mathrm{J}=4.06 \mathrm{~Hz}), 1.52-1.66(1 \mathrm{H}, \mathrm{m}), 1.80(2 \mathrm{H}, \mathrm{d}, \mathrm{J}=12.27 \mathrm{~Hz}), 2.55(2 \mathrm{H}, \mathrm{t}, \mathrm{J}=11.39 \mathrm{~Hz}), 3.04$ 
$-3.18(4 \mathrm{H}, \mathrm{m}), 3.92(3 \mathrm{H}, \mathrm{s}), 4.64(1 \mathrm{H}, \mathrm{s}), 6.59(3 \mathrm{H}, \mathrm{t}, \mathrm{J}=6.60 \mathrm{~Hz}), 7.00(2 \mathrm{H}, \mathrm{dd}, \mathrm{J}=1.80,8.37 \mathrm{~Hz}), 7.04(2 \mathrm{H}$, s).

tert-butyl 4-((5-bromo-1H-benzo[d][1,2,3]triazol-1-yl)methyl)piperidine-1-carboxylate (34d). Obtained following the general cyclisation procedure. The crude was purified using flash chromatography cyclohexane/EtOAc (1:1 $\rightarrow 0: 1)$. The compound was obtained as a brown powder. Overall yield $=55$ \%. ${ }^{1} \mathrm{H}-\mathrm{NMR}\left(400 \mathrm{MHz}, \mathrm{CDCl}_{3}\right): \delta 1.21-1.35(2 \mathrm{H}, \mathrm{m}), 1.44(9 \mathrm{H}, \mathrm{s}), 1.52-1.63(2 \mathrm{H}, \mathrm{m}), 2.15-2.27(1 \mathrm{H}$, m), $2.65(2 \mathrm{H}, \mathrm{t}, \mathrm{J}=11.90 \mathrm{~Hz}), 4.01-4.23(2 \mathrm{H}, \mathrm{m}), 4.50(2 \mathrm{H}, \mathrm{d}, \mathrm{J}=7.18 \mathrm{~Hz}), 7.40(1 \mathrm{H}, \mathrm{d}, \mathrm{J}=8.75 \mathrm{~Hz}), 7.60$ $(1 \mathrm{H}, \mathrm{q}, \mathrm{J}=3.37 \mathrm{~Hz}), 8.23(1 \mathrm{H}, \mathrm{d}, \mathrm{J}=0.73 \mathrm{~Hz}) .{ }^{13} \mathrm{C}-\mathrm{NMR}\left(100 \mathrm{MHz}, \mathrm{CDCl}_{3}\right) \delta 28.42 ; 29.78 ; 37.22 ; 53.53$; $79.72 ; 110.51 ; 117.24 ; 122.75 ; 130.88 ; 132.36 ; 146.98 ; 154.65$.

- tert-butyl 3-((5-bromo-1H-benzo[d][1,2,3]triazol-1-yl)methyl)pyrrolidine-1-carboxylate (36d)<smiles>CC(=O)N1CCC(Cn2nnc3cc(Br)ccc32)C1</smiles>

tert-butyl 3-(((4-bromo-2-nitrophenyl)amino)methyl)pyrrolidine-1-carboxylate (36b). Synthesized according to the general procedure using tert-butyl 3-(aminomethyl)pyrrolidine-1-carboxylate. Agitated at reflux temperature for $2 \mathrm{~h} 30$. The reaction media was extracted with EtOAc. After evaporation the product was obtained as an orange oil that was used without further purification in the next step. Quantitative yield. ${ }^{1} \mathrm{H}-\mathrm{NMR}\left(400 \mathrm{MHz}, \mathrm{CDCl}_{3}\right): \delta 1.46(9 \mathrm{H}, \mathrm{s}), 1.67-1.80(1 \mathrm{H}, \mathrm{m}), 2.08-$ $2.19(1 \mathrm{H}, \mathrm{m}), 2.52-2.65(1 \mathrm{H}, \mathrm{m}), 3.07-3.20(1 \mathrm{H}, \mathrm{m}), 3.21-3.70(5 \mathrm{H}, \mathrm{m}), 6.76(1 \mathrm{H}, \mathrm{d}, \mathrm{J}=9.16 \mathrm{~Hz}), 7.51$ $(1 \mathrm{H}, \mathrm{dd}, \mathrm{J}=1.59,9.08 \mathrm{~Hz}), 8.03-8.15(1 \mathrm{H}, \mathrm{m}), 8.33(1 \mathrm{H}, \mathrm{d}, \mathrm{J}=1.61 \mathrm{~Hz}) .{ }^{13 \mathrm{C}-N M R}\left(100 \mathrm{MHz}, \mathrm{CDCl}_{3}\right): \delta$ 28.42 ; 39.59; 55.39; 58.46; 79.73; 116.74; 118.52; 128.19; 131.01; 131.56; 145.57; 154.75.

tert-butyl 3-(((2-amino-4-bromophenyl)amino)methyl)pyrrolidine-1-carboxylate (36c). Obtained following the general reduction procedure as an orange oil. Quantitative yield. ${ }^{1} \mathrm{H}-\mathrm{NMR}(400 \mathrm{MHz}$, $\left.\mathrm{CDCl}_{3}\right): \delta 1.47(9 \mathrm{H}, \mathrm{s}), 1.64-1.77(1 \mathrm{H}, \mathrm{m}), 2.04-2.13(1 \mathrm{H}, \mathrm{m}), 2.43-2.56(1 \mathrm{H}, \mathrm{m}), 3.04-3.20(3 \mathrm{H}, \mathrm{m})$, $3.21-3.66(6 \mathrm{H}, \mathrm{m}), 6.49(2 \mathrm{H}, \mathrm{d}, \mathrm{J}=8.39 \mathrm{~Hz}), 6.84(1 \mathrm{H}, \mathrm{s}), 6.90(2 \mathrm{H}, \mathrm{d}, \mathrm{J}=8.14 \mathrm{~Hz})$.

tert-butyl 3-((5-bromo-1H-benzo[d][1,2,3]triazol-1-yl)methyl)pyrrolidine-1-carboxylate

(36d). Obtained following the general cyclisation procedure. Flash chromatography: Cyclohexane/EtOAc: 1:0 $\rightarrow$ 1:4. Yield: $70 \%$. ${ }^{1 \mathrm{H}-N M R}\left(400 \mathrm{MHz}, \mathrm{CDCl}_{3}\right): \delta 1.45(9 \mathrm{H}, \mathrm{s}), 1.68-1.78(1 \mathrm{H}, \mathrm{m}), 1.91-2.03(1 \mathrm{H}, \mathrm{m})$, $2.85-2.99(1 \mathrm{H}, \mathrm{m}), 3.11-3.62(5 \mathrm{H}, \mathrm{m}), 4.61(3 \mathrm{H}, \mathrm{s}), 7.41(3 \mathrm{H}, \mathrm{d}, \mathrm{J}=8.72 \mathrm{~Hz}), 7.60(3 \mathrm{H}, \mathrm{d}, \mathrm{J}=8.48 \mathrm{~Hz})$, $8.24(2 \mathrm{H}, \mathrm{s}) .{ }^{13} \mathrm{C}-\mathrm{NMR}\left(100 \mathrm{MHz}, \mathrm{CDCl}_{3}\right): \delta 28.48 ; 39.35 ; 44.62 ; 49.25 ; 50.26 ; 79.68 ; 110.27 ; 117.36$; $122.85 ; 131.03 ; 147.06 ; 154.44$.

- tert-butyl 4-((5-bromo-1H-benzo[d][1,2,3]triazol-1-yl)methyl)piperazine-1-carboxylate (38d)<smiles>CC(=O)OC(=O)N1CCN(Cn2nnc3cc(Br)ccc32)CC1</smiles>

tert-butyl 4-(((4-bromo-2-nitrophenyl)amino)methyl)piperazine-1-carboxylate (38b). Synthesized according to the general procedure using tert-butyl 4-(aminomethyl)piperazine-1-carboxylate. 
Agitated at reflux temperature for $2 \mathrm{~h} 30$. Yellow powder. Quantitative yield. ${ }^{1} \mathrm{H}-\mathrm{NMR}\left(400 \mathrm{MHz}, \mathrm{CDCl}_{3}\right)$ : $\delta 1.46(9 \mathrm{H}, \mathrm{s}), 2.47(4 \mathrm{H}, \mathrm{s}), 2.73(2 \mathrm{H}, \mathrm{t}, \mathrm{J}=5.90 \mathrm{~Hz}), 3.34(2 \mathrm{H}, \mathrm{dd}, \mathrm{J}=5.29,10.25 \mathrm{~Hz}), 3.48(4 \mathrm{H}, \mathrm{s}), 6.76(1 \mathrm{H}$, d, J=9.04 Hz), $7.00(1 \mathrm{H}, \mathrm{s}), 8.03(1 \mathrm{H}, \mathrm{d}, \mathrm{J}=9.04 \mathrm{~Hz}), 8.54(1 \mathrm{H}, \mathrm{s}) .{ }^{13} \mathrm{C}-\mathrm{NMR}\left(100 \mathrm{MHz}, \mathrm{CDCl}_{3}\right) \delta 28.51$; $37.52 ; 38.42 ; 45.18 ; 45.73 ; 49.62 ; 60.42 ; 79.54 ; 106.80 ; 115.33 ; 129.06 ; 132.36 ; 139.05 ; 144.18$; 154.49.

tert-butyl 4-(((2-amino-4-bromophenyl)amino)methyl)piperazine-1-carboxylate (38c). Obtained following the general reduction procedure as a clear oil. Quantitative yield. ${ }^{1} \mathrm{H}-\mathrm{NMR}\left(400 \mathrm{MHz}, \mathrm{CDCl}_{3}\right)$ : $\delta 1.46(9 \mathrm{H}, \mathrm{s}), 2.42(4 \mathrm{H}, \mathrm{s}), 2.68(2 \mathrm{H}, \mathrm{t}, \mathrm{J}=5.79 \mathrm{~Hz}), 3.13(2 \mathrm{H}, \mathrm{t}, \mathrm{J}=5.64 \mathrm{~Hz}), 3.34(2 \mathrm{H}, \mathrm{bs}), 3.38-3.49(5 \mathrm{H}$, m), $6.56(2 \mathrm{H}, \mathrm{d}, \mathrm{J}=8.09 \mathrm{~Hz}), 6.72(2 \mathrm{H}, \mathrm{d}, \mathrm{J}=1.85 \mathrm{~Hz}), 6.76(2 \mathrm{H}, \mathrm{dd}, \mathrm{J}=2.02,8.09 \mathrm{~Hz})$.

tert-butyl 4-((5-bromo-1H-benzo[d][1,2,3]triazol-1-yl)methyl)piperazine-1-carboxylate (38d). Obtained following the general cyclisation procedure. Flash chromatography: Cyclohexane/EtOAc 1:0 $\rightarrow$ 1:2. Yield: $80 \%$. Brown powder. ${ }^{1} \mathrm{H}-\mathrm{NMR}\left(400 \mathrm{MHz}, \mathrm{CDCl}_{3}\right): \delta 1.45(9 \mathrm{H}, \mathrm{s}), 2.46(4 \mathrm{H}, \mathrm{s}), 2.93(2 \mathrm{H}, \mathrm{t}, \mathrm{J}=6.38$ $\mathrm{Hz}), 3.37(4 \mathrm{H}, \mathrm{s}), 4.72(2 \mathrm{H}, \mathrm{t}, \mathrm{J}=6.38 \mathrm{~Hz}), 7.46(1 \mathrm{H}, \mathrm{dd}, \mathrm{J}=8.80,1.37 \mathrm{~Hz}), 7.79(1 \mathrm{H}, \mathrm{d}, \mathrm{J}=0.68 \mathrm{~Hz}), 7.92(1 \mathrm{H}$,

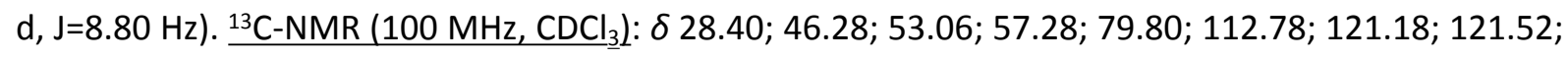
$127.57 ; 134.38 ; 144.80 ; 154.62$.

- 3-(5-bromo-1H-benzo[d][1,2,3]triazol-1-yl)propane-1,2-diol (40d).<smiles>OCC(O)Cn1nnc2cc(Br)ccc21</smiles>

3-((4-bromo-2-nitrophenyl)amino)propane-1,2-diol (40b). Synthesized according to the general procedure using 3-aminopropane-1,2-diol. Orange powder. Quantitative yield. ${ }^{1} \mathrm{H} \mathrm{NMR}(400 \mathrm{MHz}$, DMSO): 国 $3.22-3.31(1 \mathrm{H}, \mathrm{m}), 3.36-3.41(1 \mathrm{H}, \mathrm{m}), 3.42-3.52(2 \mathrm{H}, \mathrm{m}), 3.69-3.77(1 \mathrm{H}, \mathrm{m}), 4.81(1 \mathrm{H}, \mathrm{t}$, $\mathrm{J}=5.57 \mathrm{~Hz}), 5.18(1 \mathrm{H}, \mathrm{d}, \mathrm{J}=4.98 \mathrm{~Hz}), 7.08(1 \mathrm{H}, \mathrm{d}, \mathrm{J}=9.31 \mathrm{~Hz}), 7.67(1 \mathrm{H}, \mathrm{dd}, \mathrm{J}=2.14,9.24 \mathrm{~Hz}), 8.18(1 \mathrm{H}, \mathrm{d}$, $\mathrm{J}=2.30 \mathrm{~Hz}), 8.35(1 \mathrm{H}, \mathrm{t}, \mathrm{J}=4.97 \mathrm{~Hz}) .{ }^{13} \mathrm{C}$ NMR $(100 \mathrm{MHz}$, DMSO): $46.16 ; 64.07 ; 69.77 ; 105.52 ; 117.72$; $128.24 ; 131.79 ; 139.31 ; 145.11$.

3-((2-amino-4-bromophenyl)amino)propane-1,2-diol (40c). Obtained following the general reduction procedure. After evaporation, the crude extract was immediately engaged in the next step.

3-(5-bromo-1H-benzo[d][1,2,3]triazol-1-yl)propane-1,2-diol (40d). Obtained following the general cyclisation procedure. Flash chromatography: EtOAc (100\%). Yield: $80 \%$. Brown powder. ${ }^{1} \mathrm{H}$ NMR (400MHz, MeOD): $3.33(1 \mathrm{H}, \mathrm{s}), 3.62(2 \mathrm{H}, \mathrm{d}, \mathrm{J}=5.49 \mathrm{~Hz}), 4.07-4.19(1 \mathrm{H}, \mathrm{m}), 4.63-4.74(1 \mathrm{H}, \mathrm{m}), 4.86$ $(1 \mathrm{H}, \mathrm{d}, \mathrm{J}=3.52 \mathrm{~Hz}), 7.55(1 \mathrm{H}, \mathrm{q}, \mathrm{J}=3.28 \mathrm{~Hz}), 7.91(1 \mathrm{H}, \mathrm{d}, \mathrm{J}=8.82 \mathrm{~Hz}), 8.13(1 \mathrm{H}, \mathrm{s}) . \underline{13 \mathrm{C} \mathrm{NMR}(100 \mathrm{MHz},}$ MeOD): $51.06 ; 63.24 ; 70.94 ; 113.99 ; 119.79 ; 121.07 ; 127.61 ; 135.16 ; 144.10$.

2.4.2. List of $\mathrm{N}$-substituted benzotriazoles obtained starting from 4-Bromo-2-fluoro-1nitrobenzene [CAS 364-73-8]:

- 6-bromo-1-(2,2,2-trifluoroethyl)-1H-benzo[d][1,2,3]triazole (17d).<smiles>FC(F)(F)Cn1nnc2ccc(Br)cc21</smiles> 
- 5-bromo-2-nitro-N-(2,2,2-trifluoroethyl)aniline (17b). 4-Bromo-2-fluoro-1-nitrobenzene $(500 \mathrm{mg}$, $2.27 \mathrm{mmol})$ and trifluoroethanolamine. $\mathrm{HCl}$ were dissolved in anhydrous THF $(4 \mathrm{~mL})$ and DIEA $(1.16 \mathrm{~mL}$, $6.82 \mathrm{mmol}$ ) under $\mathrm{N}_{2}$. The resulting mixture was refluxed for $16 \mathrm{~h}$. After cooling to room temperature, the solvent was removed in-vacuo. Then, the residue was resuspended in $10 \mathrm{~mL}$ water and extracted 3 times with EtOAc. The $\mathrm{OL}$ was dried with $\mathrm{Na}_{2} \mathrm{SO}_{4}$ and evaporated to yield $620 \mathrm{mg}(2.07 \mathrm{mmol})$ of a bright yellow powder containing 17b. Quantitative yield. Used without further purification in the next step. ${ }^{1} \mathrm{H}$ NMR $\left(400 \mathrm{MHz}, \mathrm{CDCl}_{3}\right)$ : 回 $3.97(2 \mathrm{H}, \mathrm{qn}, \mathrm{J}=8.08 \mathrm{~Hz}), 6.94(1 \mathrm{H}, \mathrm{dd}, \mathrm{J}=1.43,9.04 \mathrm{~Hz}), 7.11(1 \mathrm{H}, \mathrm{s})$, $7.47(1 \mathrm{H}, \mathrm{d}, \mathrm{J}=8.95 \mathrm{~Hz}), 7.51(1 \mathrm{H}, \mathrm{d}, \mathrm{J}=10.16 \mathrm{~Hz}), 7.98(1 \mathrm{H}, \mathrm{t}, \mathrm{J}=8.29 \mathrm{~Hz}), 8.08(2 \mathrm{H}, \mathrm{d}, \mathrm{J}=9.05 \mathrm{~Hz}), 8.28$ $(1 \mathrm{H}, \mathrm{s}) .{ }_{13}^{13} \mathrm{CNMR}\left(100 \mathrm{MHz}, \mathrm{CDCl}_{3}\right)$ : 回 $44.67\left(\mathrm{CF}_{3}, \mathrm{q}, \mathrm{J}=34.76 \mathrm{~Hz}\right), 116.29 ; 120.91 ; 122.09$ (d, J=23.60 Hz); 125.53; 127.17 (d, J=2.33 Hz); 128.21 (d, J=13.52 Hz); 131.89; 144.42 .

- 5-bromo-N1-(2,2,2-trifluoroethyl)benzene-1,2-diamine (17c). Obtained following the general reduction procedure. After evaporation, the crude extract was immediately engaged in the next step.

- 6-bromo-1-(2,2,2-trifluoroethyl)-1H-benzo[d][1,2,3]triazole (17d) was obtained using the cyclisation general procedure. Flash chromatography purification: Cyclohexane/EtOAc (9:1). Beige powder. Yield over two steps: $68 \%$. ${ }^{1} \mathrm{H} \mathrm{NMR}\left(400 \mathrm{MHz}, \mathrm{CDCl}_{3}\right)$ : $5.21(2 \mathrm{H}, \mathrm{q}, \mathrm{J}=8.02 \mathrm{~Hz}), 7.56(1 \mathrm{H}, \mathrm{d}, \mathrm{J}=8.67 \mathrm{~Hz}), 7.78$ $(1 \mathrm{H}, \mathrm{s}), 7.99(1 \mathrm{H}, \mathrm{d}, \mathrm{J}=8.68 \mathrm{~Hz}) .{ }^{13} \mathrm{C} \mathrm{NMR}\left(100 \mathrm{MHz} \mathrm{CDCl}_{3}\right)$ : ] $49.29\left(\mathrm{~m}, \mathrm{CF}_{3}\right) ; 112.17 ; 121.28 ; 121.60$; $123.25 ; 124.06 ; 128.50 ; 134.28 ; 144.91$.

- 6-bromo-1-(cyclopropylmethyl)-1H-benzo[d][1,2,3]triazole (19d)<smiles>Brc1ccc2nnn(CC3CC3)c2c1</smiles>

-5-bromo-N-(cyclopropylmethyl)-2-nitroaniline (19b). Synthesized according to the general procedure using cyclopropylmethanamine. Quantitative yield. The product precipitated as yellow crystals that were filtrated. Then, 5-bromo-N1-(cyclopropylmethyl)benzene-1,2-diamine (19c) was obtained following the general reduction procedure. After evaporation, the crude extract was immediately engaged in the next step.

- 6-bromo-1-(cyclopropylmethyl)-1H-benzo[d][1,2,3]triazole (19d). was obtained by using the general cyclisation procedure. Reaction time $=30 \mathrm{~min}$. The compound was purified using flash chromatography (cyclohexane/EtOAc 3:1) and obtained as a dark red oil that solidified overnight. Overall yield $=51 \%$. ${ }^{1} \mathrm{H} \mathrm{NMR}\left(400 \mathrm{MHz}, \mathrm{CDCl}_{3}\right)$ : 国 $0.50(2 \mathrm{H}, \mathrm{q}, \mathrm{J}=5.21 \mathrm{~Hz}), 0.68(2 \mathrm{H}, \mathrm{q}, \mathrm{J}=6.31 \mathrm{~Hz}), 1.36-1.45(1 \mathrm{H}, \mathrm{m}), 4.48$ $(2 \mathrm{H}, \mathrm{d}, \mathrm{J}=7.13 \mathrm{~Hz}), 7.47(1 \mathrm{H}, \mathrm{dd}, \mathrm{J}=1.07,8.80 \mathrm{~Hz}), 7.77(1 \mathrm{H}, \mathrm{s}), 7.93(1 \mathrm{H}, \mathrm{d}, \mathrm{J}=8.80 \mathrm{~Hz}) .{ }^{13} \mathrm{C} \mathrm{NMR}(100 \mathrm{MHz}$, $\mathrm{CDCl}_{3}$ : : $4.51 ; 11.04 ; 53.16 ; 112.54 ; 121.20 ; 121.52 ; 127.51 ; 133.94 ; 144.92$.

- 2-(6-bromo-1H-benzo[d][1,2,3]triazol-1-yl)-N,N-dimethylethan-1-amine (25d)<smiles>CN(C)CCn1nnc2ccc(Br)cc21</smiles>

$N^{1}$-(5-bromo-2-nitrophenyl)- $N^{2}, N^{2}$-dimethylethane-1,2-diamine (25b) was synthesized according to the general procedure using $N^{1}, N^{1}$-dimethylethane-1,2-diamine. Quantitative yield. Orange powder. ${ }^{1} \mathrm{H}$ NMR $\left(400 \mathrm{MHz}, \mathrm{CDCl}_{3}\right):$ ? $2.30(6 \mathrm{H}, \mathrm{s}), 2.63(2 \mathrm{H}, \mathrm{t}, \mathrm{J}=6.08 \mathrm{~Hz}), 3.31(2 \mathrm{H}, \mathrm{dd}, \mathrm{J}=5.74,10.80 \mathrm{~Hz}), 6.73(1 \mathrm{H}$, 
q, J=3.53 Hz), $6.99(1 \mathrm{H}, \mathrm{d}, \mathrm{J}=1.14 \mathrm{~Hz}), 8.02(1 \mathrm{H}, \mathrm{d}, \mathrm{J}=9.07 \mathrm{~Hz}), 8.36(1 \mathrm{H}, \mathrm{s}) .{ }^{13} \mathrm{C} N M R\left(100 \mathrm{MHz}, \mathrm{CDCl}_{3}\right)$ : 目 $40.74 ; 45.22 ; 57.11 ; 116.60 ; 118.38 ; 128.16 ; 130.94 ; 131.51 ; 145.67$.

5-bromo-N1-(2-(dimethylamino)ethyl)benzene-1,2-diamine (25c) was obtained following the general reduction procedure. After evaporation, the crude extract was immediately engaged in the next step.

2-(6-bromo-1H-benzo[d][1,2,3]triazol-1-yl)-N,N-dimethylethan-1-amine (25d) was obtained by using the general cyclisation procedure. Flash chromatography (cyclohexane/EtOAc 3:1 $\rightarrow 0: 1$ ). Yield over two steps: 55 \%. Dark brown oil. ${ }^{1} \mathrm{H} N M R\left(400 M H z, C D C l_{3}\right)$ : ? $2.53(6 \mathrm{H}, \mathrm{s}), 3.25(2 \mathrm{H}, \mathrm{t}, \mathrm{J}=7.15 \mathrm{~Hz}), 4.90$

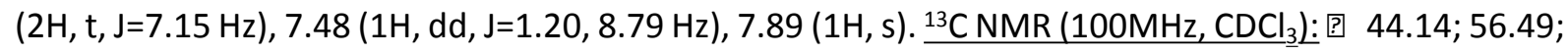
$112.64 ; 121.06 ; 122.13 ; 128.02 ; 134.10 ; 144.62 ; 176.16$.

- 6-bromo-1-((tetrahydrofuran-3-yl)methyl)-1H-benzo[d][1,2,3]triazole (27d)<smiles>Brc1ccc2nnn(CC3CCOC3)c2c1</smiles>

5-bromo-2-nitro-N-((tetrahydrofuran-3-yl)methyl)aniline (27b). was synthesized according to the general procedure using (tetrahydrofuran-3-yl)methanamine. Quantitative yield. ${ }^{1} \mathrm{H}-\mathrm{NMR}(400 \mathrm{MHz}$, $\mathrm{CDCl}_{3} L \delta 1.68-1.81(2 \mathrm{H}, \mathrm{m}), 2.14-2.25(2 \mathrm{H}, \mathrm{m}), 2.62-2.72(2 \mathrm{H}, \mathrm{m}), 3.26-3.36(4 \mathrm{H}, \mathrm{m}), 3.63-3.69$ $(2 \mathrm{H}, \mathrm{m}), 3.79(2 \mathrm{H}, \mathrm{q}, \mathrm{J}=7.86 \mathrm{~Hz}), 3.89(2 \mathrm{H}, \mathrm{q}, \mathrm{J}=5.23 \mathrm{~Hz}), 3.93-4.01(2 \mathrm{H}, \mathrm{m}), 6.78(2 \mathrm{H}, \mathrm{d}, \mathrm{J}=9.19 \mathrm{~Hz})$, $7.49(2 \mathrm{H}, \mathrm{dd}, \mathrm{J}=2.05,9.15 \mathrm{~Hz}), 8.10(1 \mathrm{H}, \mathrm{s}), 8.26(2 \mathrm{H}, \mathrm{d}, \mathrm{J}=2.19 \mathrm{~Hz}) .{ }^{13} \mathrm{C}-\mathrm{NMR}\left(100 \mathrm{MHz}, \mathrm{CDCl}_{3}\right) \delta 30.02$; $38.52 ; 46.21 ; 67.69 ; 71.22 ; 106.56 ; 115.46 ; 128.80 ; 132.13 ; 138.94 ; 144.29$.

5-bromo-N1-((tetrahydrofuran-3-yl)methyl)benzene-1,2-diamine (27c). was obtained following the general reduction procedure. After evaporation, the crude extract was immediately engaged in the next step.

6-bromo-1-((tetrahydrofuran-3-yl)methyl)-1H-benzo[d][1,2,3]triazole (27d) was obtained by using the general cyclisation procedure. Purified using flash chromatography (cyclohexane/EtOAc, 4:1). Light

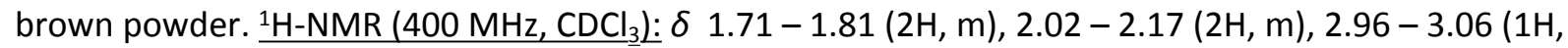
m), $3.63-3.72(1 \mathrm{H}, \mathrm{m}), 3.75-3.83(2 \mathrm{H}, \mathrm{q}, \mathrm{J}=7.46 \mathrm{~Hz}), 3.96-4.03(1 \mathrm{H}, \mathrm{m}), 4.59(2 \mathrm{H}, \mathrm{d}, \mathrm{J}=7.70 \mathrm{~Hz}), 7.44$ $(1 \mathrm{H}, \mathrm{d}, \mathrm{J}=8.75 \mathrm{~Hz}), 7.60(1 \mathrm{H}, \mathrm{t}, \mathrm{J}=4.37 \mathrm{~Hz}), 8.23(1 \mathrm{H}, \mathrm{s}) .{ }^{13} \mathrm{C}-\mathrm{NMR}\left(100 \mathrm{MHz}, \mathrm{CDCl}_{3}\right) \delta 24.86 ; 29.74 ; 39.63$; $50.63 ; 67.52 ; 70.80 ; 75.02 ; 110.36 ; 117.32 ; 122.77 ; 130.96 ; 132.11 ; 147.00$.

- 6-bromo-1-((tetrahydrofuran-2-yl)methyl)-1H-benzo[d][1,2,3]triazole (29d)<smiles>Brc1ccc2nnn(CC3CCCO3)c2c1</smiles>

-5-bromo-2-nitro-N-((tetrahydrofuran-2-yl)methyl)aniline (29b). Synthesized according to the general procedure using 2-(Aminomethyl)tetrahydrofuran. After cooling the reaction mixture to $0^{\circ} \mathrm{C}$, the precipitate was filtered and washed with cold $\mathrm{EtOH}$. The compound was obtained as a yellow powder. Yield: $50 \% .{ }^{1 \mathrm{H}-N M R}\left(400 \mathrm{MHz}, \mathrm{CDCl}_{3}\right): \delta 1.66-1.74(\mathrm{~m}, 1 \mathrm{H}), 1.94-2.01(\mathrm{~m}, 1 \mathrm{H}), 2.06-2.14(\mathrm{~m}, 1 \mathrm{H})$, $3.27-3.34(\mathrm{~m}, 1 \mathrm{H}), 3.41-3.46(\mathrm{~m}, 1 \mathrm{H}), 3.79-3.87(\mathrm{~m}, 1 \mathrm{H}), 3.93-4.00(\mathrm{~m}, 1 \mathrm{H}), 4.17-4.23(\mathrm{~m}, 1 \mathrm{H}), 6.76$ 
(d, J= 9.08Hz, 1H), $7.05(\mathrm{~s}, 1 \mathrm{H}), 8.03(\mathrm{~d}, \mathrm{~J}=8.69 \mathrm{~Hz}, 1 \mathrm{H}), 8.25$ (bs, $1 \mathrm{H}) \cdot{ }^{13} \mathrm{C}-\mathrm{NMR}\left(100 \mathrm{MHz}, \mathrm{CDCl}_{3}\right): \delta$ $25.87 ; 29.17$; $47.08 ; 68.57 ; 116.57 ; 118.75 ; 128.18 ; 131.11 ; 131.61 ; 145.92$.

-5-bromo-N-((tetrahydrofuran-2-yl)methyl)benzene-1,2-diamine (29c). Obtained following the general reduction procedure. The compound was immediately engaged in the next step.

- 6-bromo-1-((tetrahydrofuran-2-yl)methyl)-1H-benzotriazole (29d). Obtained following the general cyclisation procedure. Purified using flash chromatography (cyclohexane/EtOAc, 4:1). Light brown powder. Yield $=53 \% .{ }^{1} \mathrm{H}-\mathrm{NMR}\left(400 \mathrm{MHz}, \mathrm{CDCl}_{3}\right) \delta 1.54-1.64(\mathrm{~m}, 1 \mathrm{H}), 1.78-1.88(\mathrm{~m}, 1 \mathrm{H}), 2.03-2.11$ $(1 \mathrm{H}, \mathrm{m}), 3.67-3.70(\mathrm{~m}, 2 \mathrm{H}), 4.35-4.40(\mathrm{~m}, 1 \mathrm{H}), 4.68(\mathrm{dd}, \mathrm{J}=14.49 \mathrm{~Hz}, \mathrm{~J}=5.04 \mathrm{~Hz}, 1 \mathrm{H}), 4.80(\mathrm{dd}, \mathrm{J}=14.69$ $\mathrm{Hz}, \mathrm{J}=4.01 \mathrm{~Hz} 1 \mathrm{H}), 7.54-7.62(\mathrm{~m}, 2 \mathrm{H}), 8.19(\mathrm{~s}, 1 \mathrm{H}) .{ }^{13} \mathrm{C}-\mathrm{NMR}\left(100 \mathrm{MHz}, \mathrm{CDCl}_{3}\right) \delta 25.67 ; 28.64 ; 30.97$; $52.43 ; 68.58 ; 113.70 ; 120.86 ; 121.69 ; 127.62 ; 134.85 ; 144.81$.

- 4-(2-(6-bromo-1H-benzo[d][1,2,3]triazol-1-yl)ethyl)morpholine (31d)<smiles>Brc1ccc2nnn(CCN3CCOCC3)c2c1</smiles>

-5-bromo-N-(2-morpholinoethyl)-2-nitroaniline (31b). Synthesized according to the general procedure using 4-(2-aminoethyl)morpholine. After cooling the reaction mixture to $0^{\circ} \mathrm{C}$, the precipitate was

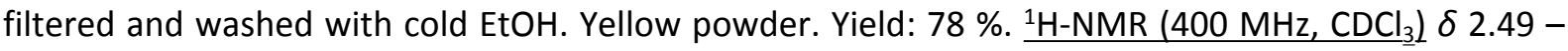
$2.56(\mathrm{~m}, 4 \mathrm{H}), 2.73(\mathrm{t}, \mathrm{J}=17.93 \mathrm{~Hz}, 2 \mathrm{H}), 3.30-3.37(\mathrm{~m}, 2 \mathrm{H}), 3.75(\mathrm{t}, \mathrm{J}=4.48 \mathrm{~Hz}, 4 \mathrm{H}), 6.75(\mathrm{~d}, \mathrm{~J}=9,08 \mathrm{~Hz}$, $1 \mathrm{H}), 7.00(\mathrm{~s}, 1 \mathrm{H}), 8.03(\mathrm{~d}, \mathrm{~J}=9.08 \mathrm{~Hz}, 1 \mathrm{H}), 8.55(\mathrm{~s}, 1 \mathrm{H}) .{ }^{13} \mathrm{C}-\mathrm{NMR}\left(100 \mathrm{MHz}, \mathrm{CDCl}_{3}\right) \delta 39.41 ; 53.13 ; 55.83$; $67.00 ; 116.72 ; 118.50 ; 128.19 ; 131.02 ; 131.55 ; 145.59$.

-5-bromo-N-(2-morpholinoethyl)benzene-1,2-diamine (31c). Obtained following the general reduction procedure. The compound was immediately engaged in the next step.

- 4-(2-(6-bromo-1H-benzo[d][1,2,3]triazol-1-yl)ethyl)morpholine (31d). Obtained following the general cyclisation procedure. The obtained compound was purified using flash chromatography (cyclohexane/EtOAc, 1:1). Light brown powder. Yield $=53 \%$. ${ }^{1 \mathrm{H}-N M R}\left(400 \mathrm{MHz}, \mathrm{CDCl}_{3}\right) \delta 7.92$ (d, J= $8.80 \mathrm{~Hz}, 1 \mathrm{H}), 7.80(\mathrm{~s}, 1 \mathrm{H}), 7.48(\mathrm{~d}, \mathrm{~J}=8.79 \mathrm{~Hz}, 1 \mathrm{H}), 4.72(\mathrm{t}, \mathrm{J}=6.38 \mathrm{~Hz}, 2 \mathrm{H}), 3.63-3.69(\mathrm{~m}, 4 \mathrm{H}), 2.92(\mathrm{t}$, $\mathrm{J}=6.39 \mathrm{~Hz}, 2 \mathrm{H}), 2.50-2.55(\mathrm{~m}, 4 \mathrm{H}) .{ }^{13} \mathrm{C}-\mathrm{NMR}\left(100 \mathrm{MHz}, \mathrm{CDCl}_{3} \mathrm{~L} \delta 46.07 ; 53.62 ; 60.42 ; 66.82 ; 112.85\right.$; $121.18 ; 121.52 ; 127.59 ; 134.37 ; 144.82$.

- tert-butyl 4-((6-bromo-1H-benzo[d][1,2,3]triazol-1-yl)methyl)piperidine-1-carboxylate (33d)<smiles>CC(C)(C)OC(=O)N1CCC(Cn2nnc3ccc(Br)cc32)CC1</smiles>

- tert-butyl 4-(((5-bromo-2-nitrophenyl)amino)methyl)piperidine-1-carboxylate (33b). Synthesized according to the general procedure using tert-butyl 4-(aminomethyl)piperidine-1-carboxylate. Agitated overnight at room temperature. An orange oil was obtained following evaporation under reduced pressure. $\underline{{ }^{1} \mathrm{H}-\mathrm{NMR}\left(400 \mathrm{MHz}, \mathrm{CDCl}_{3}\right)}: \delta 1.19-1.32(2 \mathrm{H}, \mathrm{m}), 1.47(9 \mathrm{H}, \mathrm{s}), 1.77-1.92(3 \mathrm{H}, \mathrm{m}), 2.63-2.83$ 
$(2 \mathrm{H}, \mathrm{m}), 3.19(2 \mathrm{H}, \mathrm{t}, \mathrm{J}=5.67 \mathrm{~Hz}), 4.00-4.30(2 \mathrm{H}, \mathrm{m}), 6.76(1 \mathrm{H}, \mathrm{d}, \mathrm{J}=9.09 \mathrm{~Hz}), 7.00(1 \mathrm{H}, \mathrm{s}), 8.03(1 \mathrm{H}, \mathrm{d}$, $\mathrm{J}=9.10 \mathrm{~Hz}), 8.11-8.19(1 \mathrm{H}, \mathrm{m})$.

- tert-butyl 4-((2-amino-5-bromophenyl)amino)methyl)piperidine-1-carboxylate (33c). Obtained following the general reduction procedure as a black oil with a yield of $82 \%$, which was used in the next step without further purification. ${ }^{1} \mathrm{H}-\mathrm{NMR}\left(400 \mathrm{MHz}, \mathrm{CDCl}_{3}\right): \delta 1.05-1.21(3 \mathrm{H}, \mathrm{m}), 1.38(9 \mathrm{H}, \mathrm{s})$, $1.60-1.75(3 \mathrm{H}, \mathrm{m}), 2.56-2.70(2 \mathrm{H}, \mathrm{m}), 2.87(2 \mathrm{H}, \mathrm{d}, \mathrm{J}=5.90 \mathrm{~Hz}), 3.20(2 \mathrm{H}, \mathrm{bs}), 3.44(1 \mathrm{H}, \mathrm{bs}), 3.98-4.12$ $(2 \mathrm{H}, \mathrm{m}), 6.48(2 \mathrm{H}, \mathrm{d}, \mathrm{J}=8.08 \mathrm{~Hz}), 6.61(2 \mathrm{H}, \mathrm{s}), 6.65(2 \mathrm{H}, \mathrm{d}, \mathrm{J}=8.07 \mathrm{~Hz})$.

- tert-butyl 4-((6-bromo-1H-benzo[d][1,2,3]triazol-1-yl)methyl)piperidine-1-carboxylate (33d). Obtained following the general cyclisation procedure. The obtained compound was purified using flash chromatography (cyclohexane/EtOAc, 2:1 $\rightarrow 1: 1)$. Brown powder. Yield $=84 \%$. $\underline{\text { H-NMR }(400 \mathrm{MHz}}$, $\left.\mathrm{CDCl}_{3}\right) \delta 1.23-1.36(2 \mathrm{H}, \mathrm{m}), 1.45(9 \mathrm{H}, \mathrm{s}), 1.54-1.63(2 \mathrm{H}, \mathrm{m}), 2.16-2.29(1 \mathrm{H}, \mathrm{m}), 2.61-2.73(2 \mathrm{H}, \mathrm{m})$, $4.06-4.25(2 \mathrm{H}, \mathrm{m}), 4.47(2 \mathrm{H}, \mathrm{d}, \mathrm{J}=7.00 \mathrm{~Hz}), 7.48(1 \mathrm{H}, \mathrm{d}, \mathrm{J}=7.99 \mathrm{~Hz}), 7.69(1 \mathrm{H}, \mathrm{s}), 7.94(1 \mathrm{H}, \mathrm{d}, \mathrm{J}=8.75 \mathrm{~Hz})$. ${ }^{13} \mathrm{C}-\mathrm{NMR}\left(100 \mathrm{MHz}_{,} \mathrm{CDCl}_{3}\right) \delta 28.42 ; 29.77 ; 37.12 ; 53.44 ; 79.71 ; 112.19 ; 121.33 ; 121.86 ; 127.71 ; 134.41$; $144.68 ; 154.66$.

- tert-butyl 3-((6-bromo-1H-benzo[d][1,2,3]triazol-1-yl)methyl)pyrrolidine-1-carboxylate (35d)<smiles>CC(C)(C)OC(=O)N1CCC(Cn2nnc3ccc(Br)cc32)C1</smiles>

tert-butyl 3-(((5-bromo-2-nitrophenyl)amino)methyl)pyrrolidine-1-carboxylate (35b). Synthesized according to the general procedure using tert-butyl 3-(aminomethyl)pyrrolidine-1-carboxylate. Brown oil. Quantitative yield. ${ }^{1} \mathrm{H}-\mathrm{NMR}\left(400 \mathrm{MHz}, \mathrm{CDCl}_{3}\right): \delta 1.47(9 \mathrm{H}, \mathrm{s}), 1.68-1.82(1 \mathrm{H}, \mathrm{m}), 2.09-2.20(1 \mathrm{H}$, m), $2.55-2.68(1 \mathrm{H}, \mathrm{m}), 3.09-3.20(1 \mathrm{H}, \mathrm{m}), 3.23-3.69(5 \mathrm{H}, \mathrm{m}), 6.78(1 \mathrm{H}, \mathrm{d}, \mathrm{J}=9.03 \mathrm{~Hz}), 7.01(1 \mathrm{H}, \mathrm{s})$, $8.02(1 \mathrm{H}, \mathrm{d}, \mathrm{J}=9.07 \mathrm{~Hz}), 8.08-8.12(1 \mathrm{H}, \mathrm{m})$.

tert-butyl 3-(((2-amino-5-bromophenyl)amino)methyl)pyrrolidine-1-carboxylate (35c). Obtained following the general reduction procedure. Quantitative yield. ${ }^{1} \mathrm{H}-\mathrm{NMR}\left(400 \mathrm{MHz}, \mathrm{CDCl}_{3}\right): \delta 1.47(1 \mathrm{H}$, s), $1.64-1.76(1 \mathrm{H}, \mathrm{m}), 2.02-2.12(1 \mathrm{H}, \mathrm{m}), 2.42-2.57(1 \mathrm{H}, \mathrm{m}), 3.00-3.20(3 \mathrm{H}, \mathrm{m}), 3.24-3.66(5 \mathrm{H}$, m), $6.58(1 \mathrm{H}, \mathrm{d}, \mathrm{J}=7.24 \mathrm{~Hz}), 6.71(1 \mathrm{H}, \mathrm{s}), 6.77(1 \mathrm{H}, \mathrm{d}, \mathrm{J}=7.88 \mathrm{~Hz})$.

tert-butyl 3-((6-bromo-1H-benzo[d][1,2,3]triazol-1-yl)methyl)pyrrolidine-1-carboxylate

(35d). Obtained following the general cyclisation procedure. Purified using flash chromatography (cyclohexane/EtOAc, 1:0 $\rightarrow$ 1:4). Brown powder. Yield $=70 \% .{ }^{1} \mathrm{H}-\mathrm{NMR}\left(400 \mathrm{MHz}, \mathrm{CDCl}_{3}\right): \delta 1.46(9 \mathrm{H}$, s), $1.69-1.79(1 \mathrm{H}, \mathrm{m}), 1.92-2.05(1 \mathrm{H}, \mathrm{m}), 2.87-2.98(1 \mathrm{H}, \mathrm{m}), 3.17-3.26(1 \mathrm{H}, \mathrm{d}, \mathrm{J}=4.44 \mathrm{~Hz}), 3.28-$ $3.62(3 \mathrm{H}, \mathrm{m}, \mathrm{J}=10.21 \mathrm{~Hz}), 4.60(2 \mathrm{H}, \mathrm{d}, \mathrm{J}=6.79 \mathrm{~Hz}), 7.48(1 \mathrm{H}, \mathrm{d}, \mathrm{J}=8.62 \mathrm{~Hz}), 7.72(1 \mathrm{H}, \mathrm{s}), 7.94(1 \mathrm{H}, \mathrm{d}, \mathrm{J}=8.57$ $\mathrm{Hz})$.

- tert-butyl 4-((6-bromo-1H-benzo[d][1,2,3]triazol-1-yl)methyl)piperazine-1-carboxylate (37d)<smiles>CC(C)(C)OC(=O)N1CCN(Cn2nnc3ccc(Br)cc32)CC1</smiles> 
tert-butyl 4-(((5-bromo-2-nitrophenyl)amino)methyl)piperazine-1-carboxylate (37b). Synthesized according to the general procedure using tert-butyl 4-(aminomethyl)piperazine-1-carboxylate.

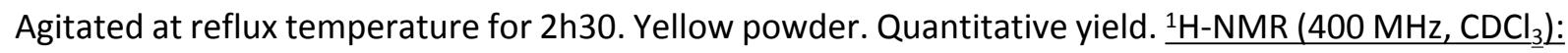
$\delta 1.47(9 \mathrm{H}, \mathrm{s}), 1.52-1.65(1 \mathrm{H}, \mathrm{m}), 1.67-1.80(1 \mathrm{H}, \mathrm{m}), 2.08-2.19(1 \mathrm{H}, \mathrm{m}), 2.59(1 \mathrm{H}, \mathrm{d}, \mathrm{J}=6.84 \mathrm{~Hz})$, $3.07-3.20(1 \mathrm{H}, \mathrm{m}), 3.21-3.69(5 \mathrm{H}, \mathrm{m}), 6.76(1 \mathrm{H}, \mathrm{d}, \mathrm{J}=9.16 \mathrm{~Hz}), 7.51(1 \mathrm{H}, \mathrm{d}, \mathrm{J}=7.80 \mathrm{~Hz}), 8.08(1 \mathrm{H}, \mathrm{s})$, $8.33(1 \mathrm{H}, \mathrm{s}) .{ }_{13}^{13} \mathrm{C}-\mathrm{NMR}\left(100 \mathrm{MHz}, \mathrm{CDCl}_{3}\right): \delta 14.21 ; 21.09 ; 28.51 ; 37.52 ; 38.42 ; 45.18 ; 45.73 ; 49.62 ; 60.42$; $79.54 ; 106.80 ; 115.33 ; 129.06 ; 132.36 ; 139.05 ; 144.18 ; 154.49$.

tert-butyl 4-(((2-amino-5-bromophenyl)amino)methyl)piperazine-1-carboxylate (37c). Obtained following the general reduction procedure. Quantitative yield. ${ }^{1} \mathrm{H}-\mathrm{NMR}\left(400 \mathrm{MHz}, \mathrm{CDCl}_{3}\right) \delta 1.46(9 \mathrm{H}$, s), $2.37-2.45(4 \mathrm{H}, \mathrm{s}), 2.65(2 \mathrm{H}, \mathrm{t}, \mathrm{J}=5.80 \mathrm{~Hz}), 3.11(2 \mathrm{H}, \mathrm{t}, \mathrm{J}=5.78 \mathrm{~Hz}), 3.38-3.47(4 \mathrm{H}, \mathrm{m}), 3.55(2 \mathrm{H}, \mathrm{br}$ s), $6.46(1 \mathrm{H}, \mathrm{d}, \mathrm{J}=8.36 \mathrm{~Hz}), 6.80(1 \mathrm{H}, \mathrm{d}, \mathrm{J}=2.08 \mathrm{~Hz}), 6.85(1 \mathrm{H}, \mathrm{dd}, \mathrm{J}=2.05,8.34 \mathrm{~Hz})$

tert-butyl 4-((6-bromo-1H-benzo[d][1,2,3]triazol-1-yl)methyl)piperazine-1-carboxylate (37d). Obtained following the general cyclisation procedure. Purified using flash chromatography (cyclohexane/EtOAc, $2: 1 \rightarrow 1: 1)$. Brown powder. Yield $=80 \%$. ${ }^{1} \mathrm{H}-\mathrm{NMR}\left(400 \mathrm{MHz}, \mathrm{CDCl}_{3} \mathrm{~L} \delta 1.45(9 \mathrm{H}, \mathrm{s}), 2.36-2.52(4 \mathrm{H}, \mathrm{m})\right.$, $2.94(2 \mathrm{H}, \mathrm{t}, \mathrm{J}=6.40 \mathrm{~Hz}), 3.25-3.47(4 \mathrm{H}, \mathrm{s}), 4.74(2 \mathrm{H}, \mathrm{t}, \mathrm{J}=6.39 \mathrm{~Hz}), 7.46(1 \mathrm{H}, \mathrm{d}, \mathrm{J}=8.75 \mathrm{~Hz}), 7.58(1 \mathrm{H}, \mathrm{dd}$, $\mathrm{J}=1.48,8.76 \mathrm{~Hz}), 8.21(1 \mathrm{H}, \mathrm{d}, \mathrm{J}=0.84 \mathrm{~Hz}) .{ }^{13} \mathrm{C}-\mathrm{NMR}\left(100 \mathrm{MHz}, \mathrm{CDCl}_{3} 2 \delta 28.39 ; 46.33 ; 53.01 ; 57.27 ; 79.81\right.$; $110.91 ; 117.13 ; 122.61 ; 130.62 ; 132.31 ; 147.06 ; 154.64$.

- 3-(6-bromo-1H-benzo[d][1,2,3]triazol-1-yl)propane-1,2-diol (39d)<smiles>OCC(O)Cn1nnc2ccc(Br)cc21</smiles>

3-((5-bromo-2-nitrophenyl)amino)propane-1,2-diol (39b). Synthesized according to the general procedure using 3-aminopropane-1,2-diol. Quantitative yield. ${ }^{1} \mathrm{H} \mathrm{NMR}$ (400MHz, DMSO): ? $3.22-3.52$ $(4 \mathrm{H}, \mathrm{m}), 3.73(1 \mathrm{H}, \mathrm{s}), 4.82(1 \mathrm{H}, \mathrm{s}), 5.20(1 \mathrm{H}, \mathrm{s}), 6.84(1 \mathrm{H}, \mathrm{dd}, \mathrm{J}=1.57,9.10 \mathrm{~Hz}), 7.29(1 \mathrm{H}, \mathrm{s}), 8.00(1 \mathrm{H}, \mathrm{d}$, $\mathrm{J}=9.10 \mathrm{~Hz}), 8.35(1 \mathrm{H}, \mathrm{t}, \mathrm{J}=4.86 \mathrm{~Hz}) .{ }^{13} \mathrm{C} \mathrm{NMR}(100 \mathrm{MHz}, \mathrm{DMSO})$ : ] 46.13; 64.04; 69.82; 117.40; 118.53; $128.53 ; 130.67 ; 131.47 ; 146.43$.

3-((2-amino-5-bromophenyl)amino)propane-1,2-diol (39c). Obtained following the general reduction procedure. After evaporation, the crude extract was immediately engaged in the next step.

3-(6-bromo-1H-benzo[d][1,2,3]triazol-1-yl)propane-1,2-diol (39d). Obtained following the general cyclisation procedure. Flash chromatography: EtOAc (100 \%). Yield: $75 \%$. Brown powder. ${ }^{1 \mathrm{H}} \mathrm{NMR}$ (400MHz, MeOD): $3.33(1 \mathrm{H}, \mathrm{s}), 3.62(1 \mathrm{H}, \mathrm{d}, \mathrm{J}=5.47 \mathrm{~Hz}), 4.11-4.19(1 \mathrm{H}, \mathrm{m}), 4.68-4.76(1 \mathrm{H}, \mathrm{m}), 4.86$ $-4.89(1 \mathrm{H}, \mathrm{m}), 7.67(1 \mathrm{H}, \mathrm{d}, \mathrm{J}=8.80 \mathrm{~Hz}), 7.81(1 \mathrm{H}, \mathrm{d}, \mathrm{J}=8.82 \mathrm{~Hz}), 8.18(1 \mathrm{H}, \mathrm{s}) .{ }^{13} \mathrm{C}$ NMR $(100 \mathrm{MHz}, \mathrm{MeOD}):$ [? $51.15 ; 63.25 ; 70.92 ; 112.56 ; 117.12 ; 120.92 ; 130.32 ; 133.15 ; 146.34$.

\subsection{Synthesis and methylation of 5-Bromotriazolopyridine}

\section{- 5-Bromotriazolopyridine (45)}

was obtained from the commercially available 5-bromopyridine-2,3-diamine (1504 mg, $8 \mathrm{mmol}$ ) using $\mathrm{NaNO}_{2}(1104 \mathrm{mg}, 16 \mathrm{mmol})$ in acetic acid $(16 \mathrm{~mL})$ using a sonication bath for $1 \mathrm{~h} .{ }^{1} \mathrm{H}-\mathrm{NMR}(400 \mathrm{MHz}$, DMSO) $\delta 8.82(1 \mathrm{H}, \mathrm{d}, \mathrm{J}=7.83 \mathrm{~Hz}) .{ }^{13} \mathrm{C}-\mathrm{NMR}(100 \mathrm{MHz}, \mathrm{DMSO}) \delta 116.93 ; 127.29 ; 150.46$. Light brown powder. Yield: $83 \%$. 
5-Bromotriazolopyridine (1194 mg, $6 \mathrm{mmol}$ ) was dissolved in $15 \mathrm{~mL}$ of $\mathrm{NaOH}_{\mathrm{aq}} 2 \mathrm{~N}$ and 1.6 eq. of $\mathrm{Me}_{2} \mathrm{SO}_{4}(9.6 \mathrm{mmol})$ were added. The reaction mixture was agitated at room temperature for $1 \mathrm{~h} 30$, until disappearance of starting material on TLC. The product gradually appeared in the form of an offwhite precipitate and contained $3 \mathrm{~N}$-methylated regioisomers. Crude yield: $96 \%$. The regioisomers were purified by flash chromatography in a gradient of cyclohexane/EtOAc $(4: 1 \rightarrow 2: 3)$.

- 6-bromo-1-methyl-1H-[1,2,3]triazolo[4,5-b]pyridine (14b)<smiles>Cn1nnc2ncc(Br)cc21</smiles>

${ }^{1} \mathrm{H}-\mathrm{NMR}(400 \mathrm{MHz}, \mathrm{DMSO}): \delta 4.33(3 \mathrm{H}, \mathrm{s}), 8.79(1 \mathrm{H}, \mathrm{d}, \mathrm{J}=1.95 \mathrm{~Hz}), 8.85(1 \mathrm{H}, \mathrm{d}, \mathrm{J}=1.98 \mathrm{~Hz}) \cdot{ }^{13} \mathrm{C}-\mathrm{NMR}(100$ $\underline{\mathrm{MHz}, \mathrm{DMSO}) \delta} 35.57 ; 118.51 ; 123.40 ; 127.39 ; 149.30 ; 155.67$. Beige powder. Purified yield : $13 \%$

- 6-bromo-3-methyl-3H-[1,2,3]triazolo[4,5-b]pyridine (15b)<smiles>Cn1nnc2cc(Br)cnc21</smiles>

${ }^{1} \mathrm{H}-\mathrm{NMR}(400 \mathrm{MHz}, \mathrm{DMSO}): \delta 4.31(3 \mathrm{H}, \mathrm{s}), 8.88(1 \mathrm{H}, \mathrm{d}, \mathrm{J}=1.89 \mathrm{~Hz}), 8.96(1 \mathrm{H}, \mathrm{d}, \mathrm{J}=1.88 \mathrm{~Hz}) \cdot{ }^{13} \mathrm{C}-\mathrm{NMR}(100$ MHz, DMSO): $\delta 33.58 ; 115.54 ; 131.06 ; 137.82 ; 144.81 ; 151.66$. White crystalline powder. Purified yield : $20.5 \%$

- 6-bromo-2-methyl-2H-[1,2,3]triazolo[4,5-b]pyridine (13b)<smiles>Cn1nc2cc(Br)cnc2n1</smiles>

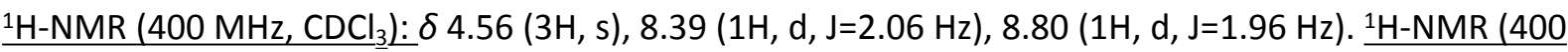
$\mathrm{MHz}, \mathrm{DMSO}): 4.55(3 \mathrm{H}, \mathrm{s}), 8.84-8.87(2 \mathrm{H}, \mathrm{m}) .{ }^{13} \mathrm{C}-\mathrm{NMR}(100 \mathrm{MHz}, \mathrm{DMSO}): \delta 44.70 ; 117.64 ; 129.47$; $137.34 ; 152.92 ; 154.16$. White crystalline powder. Purified yield: $11 \%$

A loss in purified yield is due to the lack of resolution of the glass column purification.

\subsection{General procedures of nitration (A) and reduction (B) of methylated 5- Bromobenzotriazoles}

\section{(A) Nitration}

$6.5 \mathrm{mmol}$ of $\mathrm{KNO}_{3}$ were dissolved in $3.4 \mathrm{~mL}$ of concentrated $\mathrm{H}_{2} \mathrm{SO}_{4}=$ Solution $A$.

$2 \mathrm{mmol}$ of the appropriate methylated 5-Chlorobenzotriazole was dissolved in $3.4 \mathrm{~mL}$ of concentrated $\mathrm{H}_{2} \mathrm{SO}_{4}=$ Solution $B$.

Solution $A$ was added dropwise into solution $B$. The reaction mixture was agitated for $1 \mathrm{~h} 30$ at $50^{\circ} \mathrm{C}$, until completion of the reaction. After cooling to room temperature, it was poured on ice and the precipitate was filtrated. The powder was washed until neutrality. In accordance with the literature nitration of 5-chloro-2-methyl-2H-benzo[d][1,2,3]triazole (AK-2-150) led to the formation of a second 
di-nitrated product: 5-bromo-2-methyl-4,6-dinitro-2H-benzo[d][1,2,3]triazole in addition to the mononitrated 5-chloro-2-methyl-4-nitro-2H-benzo[d][1,2,3]triazole.

\subsubsection{Nitrated N-methylbenzotriazoles}

- 5-chloro-2-methyl-4-nitro-2H-benzo[d][1,2,3]triazole (10b)

Obtained following the general procedure $A$ using $7 \mathrm{~b}, 77 \%$ yield, white powder. ${ }^{1} \mathrm{H}-\mathrm{NMR}(400$ $\mathrm{MHz}, \mathrm{CDCl}_{3} \mathrm{~L} \delta 8.01(\mathrm{~d}, \mathrm{~J}=9.03 \mathrm{~Hz}, 1 \mathrm{H}), 7.49(\mathrm{~d}, \mathrm{~J}=9.03 \mathrm{~Hz}, 1 \mathrm{H}), 4.58(\mathrm{~s}, 3 \mathrm{H}) .{ }^{13} \mathrm{C}-\mathrm{NMR}\left(100 \mathrm{MHz}, \mathrm{CDCl}_{3}\right)$ $\delta 44.09 ; 118.83 ; 122.80 ; 125.95 ; 128.53 ; 138.26 ; 144.28$.

\section{- 5-chloro-2-methyl-4,6-dinitro-2H-benzo[d][1,2,3]triazole}

Obtained as a side product following the general procedure $A$ using $7 \mathbf{b}, 23 \%$ yield, white

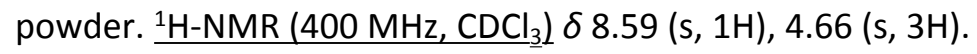

- 6-chloro-1-methyl-4-nitro-1H-benzo[d][1,2,3]triazole (11b)

Obtained following the general procedure $A$ using $\mathbf{8 b}$, quantitative yield, white powder. ${ }^{1} \mathrm{H}-$ NMR $\left(400 \mathrm{MHz}, \mathrm{CDCl}_{3}\right) \delta 4.26(3 \mathrm{H}, \mathrm{s}), 7.50(1 \mathrm{H}, \mathrm{d}, \mathrm{J}=8.83 \mathrm{~Hz}), 8.17(1 \mathrm{H}, \mathrm{d}, \mathrm{J}=8.83 \mathrm{~Hz}) .{ }^{13} \mathrm{C}-\mathrm{NMR}(100$ $\left.\mathrm{MHz}_{1} \mathrm{CDCl}_{3}\right) \delta 36.20 ; 123.73 ; 125.84 ; 126.01 ; 126.55 ; 146.94$.

- 5-chloro-1-methyl-4 nitro-1H-benzo[d][1,2,3]triazole (12b)

Obtained following the general procedure $A$ using $A K-170$, quantitative yield, white powder. $\underline{{ }^{1} \mathrm{H}-\mathrm{NMR}\left(400 \mathrm{MHz}, \mathrm{CDCl}_{3}\right)} \delta 4.39(3 \mathrm{H}, \mathrm{s}), 7.61(1 \mathrm{H}, \mathrm{d}, \mathrm{J}=8.84 \mathrm{~Hz}), 7.69(1 \mathrm{H}, \mathrm{d}, \mathrm{J}=8.84 \mathrm{~Hz}) .{ }^{13} \mathrm{C}-\mathrm{NMR}(100$

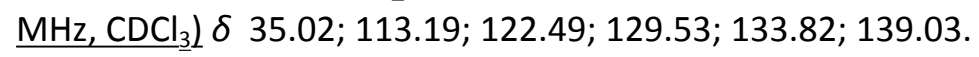

\section{(B) Reduction}

The product of nitration ( $2 \mathrm{mmol}$ ) was reduced using 10 equi. of iron powder $\left(\mathrm{Fe}_{(\mathrm{s})}\right)$ in a mixture of $3 \mathrm{~mL}$ of saturated $\mathrm{NH}_{4} \mathrm{Cl}$ and $11 \mathrm{~mL} \mathrm{EtOH}$ under reflux for $30 \mathrm{~min}$. to 16h. Upon completion, the reaction medium is filtrated over a celite pad, washed with EtOAc. After extraction, the $\mathrm{OL}$ is dried with $\mathrm{Na}_{2} \mathrm{SO}_{4}$ and concentrated in-vacuo.

\subsubsection{N-methylbenzotriazol-4-amines}

- 5-chloro-2-methyl-2H-benzo[d][1,2,3]triazol-4-amine (10c).

Obtained in quantitative yield, starting from 10b, as a powder. ${ }^{1} \mathrm{H}-\mathrm{NMR}$ ( $400 \mathrm{MHz}, \mathrm{DMSO}$ ): $\delta 4.45$ (s, 3H), 6.01 (bs, 1H), 7.02 (d, J= $8.92 \mathrm{~Hz}, 1 \mathrm{H}), 7.20$ (d, J= $8.93 \mathrm{~Hz}, 1 \mathrm{H}) .{ }^{13} \mathrm{C}-\mathrm{NMR}(100 \mathrm{MHz}, \mathrm{DMSO}) \delta 43.54$; $104.77 ; 107.79 ; 128.91 ; 135.31 ; 136.91 ; 144.02$.

- 6-chloro-1-methyl-1H-benzo[d][1,2,3]triazol-4-amine (11c).

Obtained in quantitative yield, starting from $11 \mathrm{~b}$, as a yellow powder. ${ }^{1} \mathrm{H}-\mathrm{NMR}\left(400 \mathrm{MHz}, \mathrm{CDCl}_{3}\right) \delta 4.45$ (bs, 2H), $4.52(\mathrm{~s}, 3 \mathrm{H}) 7.20(\mathrm{~d}, \mathrm{~J}=8.60 \mathrm{~Hz}, 1 \mathrm{H}), 7.34(\mathrm{~d}, \mathrm{~J}=8.60 \mathrm{~Hz}, 1 \mathrm{H}) .{ }^{13} \mathrm{C}-\mathrm{NMR}\left(100 \mathrm{MHz}, \mathrm{CDCl}_{3}\right) \delta 36.74$; $110.22 ; 116.31 ; 125.48 ; 126.08 ; 128.38 ; 146.49$.

- 5-chloro-1-methyl-1H-benzo[d][1,2,3]triazol-4-amine (12c).

Obtained starting from 12b. Purified using a DCM $+1 \% \mathrm{MeOH}$ flash column. Yield $=73 \% .{ }^{1} \mathrm{H}-\mathrm{NMR}(400$

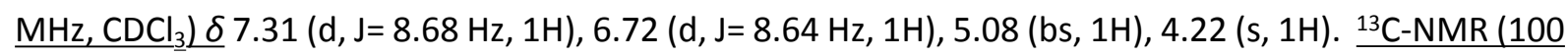
$\left.\mathrm{MHz}, \mathrm{CDCl}_{3}\right) \delta 34.35 ; 97.47 ; 109.71 ; 129.19 ; 133.51 ; 135.25 ; 136.99$. 


\subsection{General procedures for the Suzuki-Miyaura cross-coupling reaction}

1,4-Dioxane and $\mathrm{K}_{3} \mathrm{PO}_{4}$ solution in water were degassed by atmosphere exchange with nitrogen in a sonication bath for 45 minutes. We found that he use of $\mathrm{Pd}(\mathrm{dppf}) \mathrm{Cl}_{2}$ as catalyst significantly increases the yield as compared to $\mathrm{Pd}(\mathrm{OAc})_{2}$. The cross-coupling procedure was adapted from DM Knapp et al. J. Am. Chem. Soc. 2009, 131, 20, 6961-6963.

\section{(A) Using a pinacol boronic ester}

Aryl-halide $(1 \mathrm{mmol})$ and the boronic ester $(1.6 \mathrm{mmol})$ are introduced into a $25 \mathrm{ml}$ 2-neck roundbottom flask under inert atmosphere, then $\mathrm{Pd}(\mathrm{dppf}) \mathrm{Cl}_{2}(5 \mathrm{~mol} \%)$ and SPhos $(10 \mathrm{~mol} \%)$ are added. 10 $\mathrm{mL}$ of dioxane were introduced through the septum using a syringe. The reaction was left under agitation for 10 minutes until dissolution of reactants, before adding $2 \mathrm{~mL}$ of distilled water containing $\mathrm{K}_{3} \mathrm{PO}_{4}(3.5 \mathrm{mmol})$. The reaction was heated and left under agitation 3 to $16 \mathrm{~h}$ at $70^{\circ} \mathrm{C}$.

(B) Using a MIDA boronic ester

Aryl-halide $(1 \mathrm{mmol})$ and the boronic ester $(1.2 \mathrm{mmol})$ are introduced into a $25 \mathrm{ml}$ 2-neck roundbottom flask under inert atm., then $\mathrm{Pd}(\mathrm{dppf}) \mathrm{Cl}_{2}(5 \mathrm{~mol} \%)$ and SPhos $(10 \mathrm{~mol} \%)$ are added. $10 \mathrm{~mL}$ of dioxane were introduced through the septum using a syringe. The reaction was left under agitation for 10 minutes until dissolution of reactants, before adding $2 \mathrm{~mL}$ of distilled water containing $\mathrm{K}_{3} \mathrm{PO}_{4}(8.25$ $\mathrm{mmol})$.

The reaction was heated and left under agitation over-night at $70^{\circ} \mathrm{C}$.

\subsection{General deprotection procedures.}

2.8.1. Phenylsulfonyl $\left(\mathrm{SO}_{2} \mathrm{Ph}\right)$ deprotection

Two different deprotection protocols were used for removing N-phenylsulfonyl present on the indole of cross-coupled compounds depending on the stability of moieties present on the molecule.

(A) Strong deprotection conditions

$0.5 \mathrm{mmol}$ of starting compound were dissolved in $4 \mathrm{~mL}$ of a 1:1 solution of $\mathrm{MeOH} / \mathrm{H}_{2} \mathrm{O}$ containing 2.5 $\mathrm{M}$ of $\mathrm{KOH}$. The resulting mixture was refluxed until completion of the reaction ( $30 \mathrm{~min}$. $-48 \mathrm{~h}$.).

(B) Mild deprotection conditions

$0.5 \mathrm{mmol}$ of starting compound were dissolved in $4 \mathrm{~mL}$ of a $1 \mathrm{M}$ solution of TBAF in THF. The resulting mixture was refluxed until completion of the reaction ( $2 \mathrm{~h} .-48 \mathrm{~h}$.).

\subsection{2. tert-butyloxycarbonyl (Boc) deprotection (C)}

$0.5 \mathrm{mmol}$ of starting compound were dissolved in $4 \mathrm{~mL}$ of a 1:1 solution of $\mathrm{MeOH} / \mathrm{H} 2 \mathrm{O}$ containing $4 \mathrm{M}$ of $\mathrm{KOH}$. The resulting mixture was refluxed until completion of the reaction (30 min. $-48 \mathrm{~h}$.).

\subsection{Products of cross-coupling and deprotection.}

\subsubsection{List of compounds obtained following cross-coupling procedure A (using $\mathbf{4 3}$ )}

The following compounds were synthesized according to the general coupling procedure and then deprotected using the appropriate deprotection procedure.

- 6-(1H-indol-3-yl)-1H-benzo[d][1,2,3]triazole (6) 
<smiles>c1ccc2c(-c3ccc4nn[nH]c4c3)c[nH]c2c1</smiles>

1-(4-methoxybenzyl)-6-(1-(phenylsulfonyl)-1H-indol-3-yl)-1H-benzo[d][1,2,3]triazole was obtained after coupling between compounds $\mathbf{4 3}$ and $\mathbf{4 4}$. Purified using flash chromatography (Cyclohexane/ EtOAc 3:1). The obtained white powder underwent general deprotection procedure 2.8.1.A. to afford 6-(1H-indol-3-yl)-1-(4-methoxybenzyl)-1H-benzo[d][1,2,3]triazole, which was deprotected in pure TFA at reflux temperature. The reaction was left for $16 \mathrm{~h}$, after which only around $20 \%$ of the reactant were deprotected a flash chromatography, the rest was starting material. Purification by flash chromatography (cyclohexane/EtOAc) was performed. 6-(1H-indol-3-yl)-1-(4-methoxybenzyl)-1Hbenzo[d][1,2,3]triazole was deprotected again under the same conditions. This procedure was

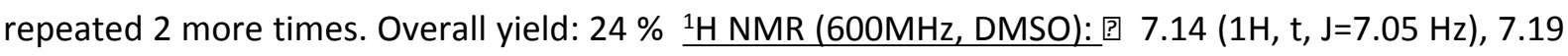
$(1 \mathrm{H}, \mathrm{t}, \mathrm{J}=7.10 \mathrm{~Hz}), 7.48(1 \mathrm{H}, \mathrm{d}, \mathrm{J}=8.02 \mathrm{~Hz}), 7.76(1 \mathrm{H}, \mathrm{dd}, \mathrm{J}=1.34,8.59 \mathrm{~Hz}), 7.82(1 \mathrm{H}, \mathrm{d}, \mathrm{J}=2.53 \mathrm{~Hz}), 7.94$ $(1 \mathrm{H}, \mathrm{d}, \mathrm{J}=7.90 \mathrm{~Hz}), 7.97(1 \mathrm{H}, \mathrm{d}, \mathrm{J}=8.58 \mathrm{~Hz}), 8.04(1 \mathrm{H}, \mathrm{s}), 11.45(1 \mathrm{H}, \mathrm{s}) \cdot{ }^{13} \mathrm{C}$ NMR $(150 \mathrm{MHz}, \mathrm{DMSO}):$ ? $110.29 ; 112.56 ; 115.88 ; 116.63 ; 119.35 ; 120.30 ; 122.06 ; 124.70 ; 125.48 ; 134.01 ; 137.43$. HRMS: $\mathrm{m} / \mathrm{z}$ $(\mathrm{El}+)$, calculated for: $\mathrm{C}_{14} \mathrm{H}_{10} \mathrm{~N}_{4} 235.0939[\mathrm{M}]^{+}$, found: $235.0639[\mathrm{M}]^{+}$. Melting temp. range: $120-124^{\circ} \mathrm{C}$.

- 5-(1H-indol-3-yl)-2-methyl-2H-benzo[d][1,2,3]triazole (7)<smiles>Cn1nc2ccc(-c3c[nH]c4ccccc34)cc2n1</smiles>

2-methyl-5-(1-(phenylsulfonyl)-1H-indol-3-yl)-2H-benzo[d][1,2,3]triazole was obtained after coupling 43 with 7b. Purified by flash chromatography (Cyclohexane/EtOAc: 3:1 $\rightarrow$ 2:1). Off white powder. Yield: 76\%. ${ }^{1} \mathrm{H}$ NMR $\left(400 \mathrm{MHz} \mathrm{CDCl}_{3}\right)$ : $4.54(3 \mathrm{H}, \mathrm{s}), 7.32(1 \mathrm{H}, \mathrm{t}, \mathrm{J}=7.51 \mathrm{~Hz}), 7.40(1 \mathrm{H}, \mathrm{t}, \mathrm{J}=7.51 \mathrm{~Hz}), 7.46$ $(2 \mathrm{H}, \mathrm{t}, \mathrm{J}=7.69 \mathrm{~Hz}), 7.56(1 \mathrm{H}, \mathrm{t}, \mathrm{J}=7.41 \mathrm{~Hz}), 7.62(1 \mathrm{H}, \mathrm{dd}, \mathrm{J}=8.82,1.09 \mathrm{~Hz}), 7.79(1 \mathrm{H}, \mathrm{s}), 7.83(1 \mathrm{H}, \mathrm{d}, \mathrm{J}=7.86$

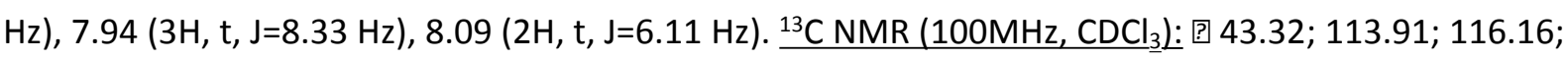
$118.44 ; 120.42 ; 123.38 ; 123.85 ; 125.21 ; 126.89 ; 127.43 ; 129.21 ; 129.41 ; 131.24 ; 134.01 ; 135.54$; $138.12 ; 144.06 ; 145.03$.

2-methyl-5-(1-(phenylsulfonyl)-1H-indol-3-yl)-2H-benzo[d][1,2,3]triazole was deprotected following general procedure $A$ to afford 7. Purified by flash chromatography (DCM/MeOH: 1:0 $\rightarrow$ 9:1). Off white powder. Yield: $56 \%$. ${ }^{1} \mathrm{H} \mathrm{NMR}\left(600 \mathrm{MHz}, \mathrm{CDCl}_{3}\right)$ : 回 $4.54(3 \mathrm{H}, \mathrm{s}), 7.22-7.25(1 \mathrm{H}, \mathrm{m}), 7.27-7.30(1 \mathrm{H}, \mathrm{m})$, $7.47(1 \mathrm{H}, \mathrm{dd}, \mathrm{J}=2.77,5.30 \mathrm{~Hz}), 7.73(1 \mathrm{H}, \mathrm{dd}, \mathrm{J}=1.47,8.82 \mathrm{~Hz}), 7.91(1 \mathrm{H}, \mathrm{d}, \mathrm{J}=8.81 \mathrm{~Hz}), 8.01(1 \mathrm{H}, \mathrm{d}, \mathrm{J}=7.93$ $\mathrm{Hz}), 8.11(1 \mathrm{H}, \mathrm{s}), 8.34(1 \mathrm{H}, \mathrm{s}) .{ }^{13} \mathrm{C}$ NMR $\left(150 \mathrm{MHz}, \mathrm{CDCl}_{3}\right):$ ? $43.16 ; 111.53 ; 114.50 ; 117.93 ; 118.15$; $119.77 ; 120.64 ; 122.31 ; 122.70 ; 125.72 ; 127.92 ; 133.90 ; 136.73 ; 143.56 ; 145.45$. HRMS: $\mathrm{m} / \mathrm{z}$ (EI+), calculated for: $\mathrm{C}_{15} \mathrm{H}_{12} \mathrm{~N}_{4} 249.1096[\mathrm{M}]^{+}$, found: $249.1132[\mathrm{M}]^{+}$. Melting temp. range: $157-160{ }^{\circ} \mathrm{C}$.

- 5-(1H-indol-3-yl)-2-methyl-2H-benzo[d][1,2,3]triazol-4-amine (10) 
<smiles>Cn1nc2ccc(-c3c[nH]c4ccccc34)c(N)c2n1</smiles>

2-methyl-5-(1-(phenylsulfonyl)-1H-indol-3-yl)-2H-benzo[d][1,2,3]triazol-4-amine was obtained after coupling 43 with 10c. Purified by flash chromatography (Cyclohexane/EtOAc: 3:1 $\rightarrow$ 1:1). Yield: $64 \%$. $\underline{1}$ H NMR $\left(400 \mathrm{MHz}_{1} \mathrm{CDCl}_{3}\right)$ : 回 $4.46(2 \mathrm{H}, \mathrm{s}) 4.51(3 \mathrm{H}, \mathrm{s}), 7.23-7.30(4 \mathrm{H}, \mathrm{m}), 7.39(1 \mathrm{H}, \mathrm{t}, \mathrm{J}=7.62 \mathrm{~Hz}), 7.50$ $(3 \mathrm{H}, \mathrm{q}, \mathrm{J}=8.21 \mathrm{~Hz}), 7.58(1 \mathrm{H}, \mathrm{t}, \mathrm{J}=7.41 \mathrm{~Hz}), 7.72(1 \mathrm{H}, \mathrm{s}), 7.97(2 \mathrm{H}, \mathrm{d}, \mathrm{J}=7.53 \mathrm{~Hz}), 8.10(1 \mathrm{H}, \mathrm{d}, \mathrm{J}=8.33 \mathrm{~Hz})$. ${ }^{13} \mathrm{C} \mathrm{NMR}\left(100 \mathrm{MHz}, \mathrm{CDCl}_{3}\right)$ : ? 43.16; 106.24; 108.69; 113.87; 120.74; 121.11; 123.58; 124.26; 125.22; $126.90 ; 129.42 ; 129.92 ; 130.29 ; 134.01 ; 134.83 ; 135.24$.

2-methyl-5-(1-(phenylsulfonyl)-1H-indol-3-yl)-2H-benzo[d][1,2,3]triazol-4-amine was deprotected following general procedure $A$ to afford 10. Purified by flash chromatography (Cyclohexane/EtOAc: $2: 1 \rightarrow 0: 1)$. Yield: $50 \%$. ${ }^{1} \mathrm{H}$ NMR $(600 \mathrm{MHz}, \mathrm{DMSO}):$ ? $4.52(3 \mathrm{H}, \mathrm{s}), 5.26(2 \mathrm{H}, \mathrm{s}), 7.09(1 \mathrm{H}, \mathrm{t}, \mathrm{J}=7.15 \mathrm{~Hz})$, $7.17(1 \mathrm{H}, \mathrm{d}, \mathrm{J}=8.59 \mathrm{~Hz}), 7.21(1 \mathrm{H}, \mathrm{t}, \mathrm{J}=7.50 \mathrm{~Hz}), 7.36(1 \mathrm{H}, \mathrm{d}, \mathrm{J}=8.59 \mathrm{~Hz}), 7.52(2 \mathrm{H}, \mathrm{dd}, \mathrm{J}=3.12,8.06 \mathrm{~Hz})$, $7.56(1 \mathrm{H}, \mathrm{d}, \mathrm{J}=2.43 \mathrm{~Hz}), 11.38(1 \mathrm{H}, \mathrm{s}) .{ }^{13} \mathrm{C}$ NMR $(150 \mathrm{MHz}, \mathrm{DMSO})$ : 回 43.39; 104.40; 111.10; 112.30; $112.91 ; 119.48 ; 119.72 ; 121.80 ; 124.54 ; 126.57 ; 131.32 ; 135.09 ; 136.80 ; 137.64 ; 144.43$.

. HRMS: $\mathrm{m} / \mathrm{z}(\mathrm{El}+)$, calculated for: $\mathrm{C}_{15} \mathrm{H}_{13} \mathrm{~N}_{5} 264.1205$ [M] $]^{+}$, found: 264.1242 [M] $]^{+}$. Melting temp. range: $135-139^{\circ} \mathrm{C}$.

- 6-(1H-indol-3-yl)-1-methyl-1H-benzo[d][1,2,3]triazole (8)<smiles>Cn1nnc2ccc(-c3c[nH]c4ccccc34)cc21</smiles>

1-methyl-6-(1-(phenylsulfonyl)-1H-indol-3-yl)-1H-benzo[d][1,2,3]triazole was obtained after coupling $\mathbf{4 3}$ with 8b. Purified by flash chromatography (Cyclohexane/EtOAc: $2: 1 \rightarrow 1: 1$ ). Off white powder. Yield: $87 \%$. ${ }^{1} \mathrm{H}$ NMR $(400 \mathrm{MHz}, \mathrm{DMSO})$ : 回 $4.35(3 \mathrm{H}, \mathrm{s}, 7.34(1 \mathrm{H}, \mathrm{t}, \mathrm{J}=7.20 \mathrm{~Hz}), 7.42(1 \mathrm{H}, \mathrm{t}, \mathrm{J}=7.38 \mathrm{~Hz}), 7.48$ $(2 \mathrm{H}, \mathrm{t}, \mathrm{J}=7.69 \mathrm{~Hz}), 7.58(1 \mathrm{H}, \mathrm{t}, \mathrm{J}=7.45 \mathrm{~Hz}), 7.62(1 \mathrm{H}, \mathrm{dd}, \mathrm{J}=1.24,8.62 \mathrm{~Hz}), 7.71(1 \mathrm{H}, \mathrm{s}), 7.78-7.83(2 \mathrm{H}$, m), $7.96(2 \mathrm{H}, \mathrm{d}, \mathrm{J}=7.44 \mathrm{~Hz}), 8.08-8.15(2 \mathrm{H}, \mathrm{m}) .{ }^{13} \mathrm{C} \mathrm{NMR}(100 \mathrm{MHz}, \mathrm{DMSO}):$ ? 34.35; 107.78; 113.97; $120.21 ; 120.45 ; 123.45 ; 123.68 ; 123.95 ; 124.72 ; 125.38 ; 126.92 ; 129.04 ; 129.47 ; 132.62 ; 134.09$; $134.12 ; 135.48 ; 138.02 ; 145.44$.

1-methyl-6-(1-(phenylsulfonyl)-1H-indol-3-yl)-1H-benzo[d][1,2,3]triazole was deprotected following general procedure $A$ to afford 8 . Purified by flash chromatography (DCM/MeOH: 1:0 $\rightarrow$ 9:1). Off white powder. Yield: $79 \%$. ${ }^{1} \mathrm{H}$ NMR $(400 \mathrm{MHz}, \mathrm{DMSO})$ : $4.36(3 \mathrm{H}, \mathrm{s}), 7.13-7.24(2 \mathrm{H}, \mathrm{m}), 7.50(1 \mathrm{H}, \mathrm{d}, \mathrm{J}=7.83$ $\mathrm{Hz}), 7.78(2 \mathrm{H}, \mathrm{d}, \mathrm{J}=8.88 \mathrm{~Hz}), 7.88(1 \mathrm{H}, \mathrm{d}, \mathrm{J}=2.24 \mathrm{~Hz}), 8.01-8.10(3 \mathrm{H}, \mathrm{m}), 11.54(1 \mathrm{H}, \mathrm{s}) .{ }^{13} \mathrm{C} \mathrm{NMR}(100 \mathrm{MHz}$, DMSO): 回 34.53; 106.63; 112.56; 115.63; 119.56; 119.73; 120.42; 122.20; 124.57; 125.21; 125.34; 134.73; 135.65; 137.47; 144.00. HRMS: $\mathrm{m} / \mathrm{z}(\mathrm{El}+)$, calculated for: $\mathrm{C}_{15} \mathrm{H}_{12} \mathrm{~N}_{4} 249.1096[\mathrm{M}]^{+}$, found: $249.1135[\mathrm{M}]^{+}$. Melting temp. range: $230-234^{\circ} \mathrm{C}$.

- 6-(1H-indol-3-yl)-1-methyl-1H-benzo[d][1,2,3]triazol-7-amine (11) 
<smiles>Cn1nnc2ccc(-c3c[nH]c4ccccc34)c(N)c21</smiles>

1-methyl-6-(1-(phenylsulfonyl)-1H-indol-3-yl)-1H-benzo[d][1,2,3]triazol-7-amine was obtained after coupling 43 with 11c. Purified by flash chromatography (Cyclohexane/EtOAc 1:0 $\rightarrow$ 1:2). Yield: $39 \%$. $\underline{1 H}$ NMR (400MHz, DMSO): 国 $4.08(2 \mathrm{H}, \mathrm{s}), 4.59(3 \mathrm{H}, \mathrm{s}), 7.18(1 \mathrm{H}, \mathrm{d}, \mathrm{J}=8.52 \mathrm{~Hz}), 7.29(1 \mathrm{H}, \mathrm{d}, \mathrm{J}=7.41 \mathrm{~Hz}), 7.40$ $(1 \mathrm{H}, \mathrm{d}, \mathrm{J}=8.04 \mathrm{~Hz}), 7.44(1 \mathrm{H}, \mathrm{d}, \mathrm{J}=7.88 \mathrm{~Hz}), 7.47-7.55(3 \mathrm{H}, \mathrm{m}), 7.60(1 \mathrm{H}, \mathrm{t}, \mathrm{J}=7.42 \mathrm{~Hz}), 7.70(1 \mathrm{H}, \mathrm{s}), 7.97$ $(2 \mathrm{H}, \mathrm{d}, \mathrm{J}=7.52 \mathrm{~Hz}), 8.10(1 \mathrm{H}, \mathrm{d}, \mathrm{J}=8.33 \mathrm{~Hz}) .{ }^{13} \mathrm{C} \mathrm{NMR}(100 \mathrm{MHz}, \mathrm{DMSO}):$ ? $36.96 ; 110.23 ; 113.89 ; 114.63$; $119.82 ; 120.86 ; 123.77 ; 124.70 ; 125.48 ; 126.92 ; 127.94 ; 129.50 ; 129.75 ; 129.81 ; 134.15 ; 135.18$; 138.03 .

1-methyl-6-(1-(phenylsulfonyl)-1H-indol-3-yl)-1H-benzo[d][1,2,3]triazol-7-amine was deprotected following general procedure $A$ to afford 11. Purified by flash chromatography (Cyclohexane/EtOAc 2:1 $\rightarrow 0: 1)$. ${ }^{1} \mathrm{H} \mathrm{NMR}(400 \mathrm{MHz}, \mathrm{DMSO})$ : ] $4.55(3 \mathrm{H}, \mathrm{s}), 4.99(2 \mathrm{H}, \mathrm{s}), 7.06(1 \mathrm{H}, \mathrm{t}, \mathrm{J}=7.44 \mathrm{~Hz}), 7.18(1 \mathrm{H}, \mathrm{t}, \mathrm{J}=7.52$ $\mathrm{Hz}), 7.23(1 \mathrm{H}, \mathrm{d}, \mathrm{J}=8.47 \mathrm{~Hz}), 7.33(1 \mathrm{H}, \mathrm{d}, \mathrm{J}=8.46 \mathrm{~Hz}), 7.44(1 \mathrm{H}, \mathrm{d}, \mathrm{J}=7.90 \mathrm{~Hz}), 7.47-7.57(2 \mathrm{H}, \mathrm{m}) .{ }^{13} \mathrm{C}$ NMR (100MHz, DMSO): 回 37.25; 107.93; 112.29; 112.41; 117.09; 119.70; 121.99; 125.06; 126.27; 126.51; 128.81; 131.19; 136.82; 146.79. HRMS: $\mathrm{m} / \mathrm{z}(\mathrm{El}+)$, calculated for: $\mathrm{C}_{15} \mathrm{H}_{13} \mathrm{~N}_{5} 264.1205$ [M] ${ }^{+}$, found: $264.1242[\mathrm{M}]^{+}$. Melting temp. range: $299-304{ }^{\circ} \mathrm{C}$.

- 5-(1H-indol-3-yl)-1-methyl-1H-benzo[d][1,2,3]triazole (9)<smiles>Cn1nnc2cc(-c3c[nH]c4ccccc34)ccc21</smiles>

1-methyl-5-(1-(phenylsulfonyl)-1H-indol-3-yl)-1H-benzo[d][1,2,3]triazole was obtained after coupling 43 with 9b. Purified by flash chromatography (Cyclohexane/EtOAc 3:1 $\rightarrow 0: 1$ ). Yield: $67 \%$. ${ }^{1 \mathrm{H}}$ NMR $\left(400 \mathrm{MHz} \mathrm{CDCl}_{3}\right)$ : 回 $4.34(3 \mathrm{H}, \mathrm{s}), 7.32(1 \mathrm{H}, \mathrm{t}, \mathrm{J}=7.53 \mathrm{~Hz}), 7.40(1 \mathrm{H}, \mathrm{t}, \mathrm{J}=7.64 \mathrm{~Hz}), 7.46(2 \mathrm{H}, \mathrm{t}, \mathrm{J}=7.69 \mathrm{~Hz})$, $7.55(1 \mathrm{H}, \mathrm{t}, \mathrm{J}=7.43 \mathrm{~Hz}), 7.60(1 \mathrm{H}, \mathrm{d}, \mathrm{J}=8.55 \mathrm{~Hz}), 7.72(1 \mathrm{H}, \mathrm{dd}, \mathrm{J}=1.12,8.55 \mathrm{~Hz}), 7.76(1 \mathrm{H}, \mathrm{s}), 7.80(1 \mathrm{H}, \mathrm{d}$, $\mathrm{J}=7.87 \mathrm{~Hz}), 7.95(2 \mathrm{H}, \mathrm{d}, \mathrm{J}=7.63 \mathrm{~Hz}), 8.09(1 \mathrm{H}, \mathrm{d}, \mathrm{J}=8.30 \mathrm{~Hz}), 8.26(1 \mathrm{H}, \mathrm{s}) \cdot{ }^{13} \mathrm{C}$ NMR $\left(100 \mathrm{MHz}, \mathrm{CDCl}_{3}\right)$ : 回 $34.42 ; 109.78 ; 113.90 ; 118.56 ; 120.24 ; 123.24 ; 123.46 ; 123.90 ; 125.26 ; 126.88 ; 128.13 ; 129.07$; $129.21 ; 129.43 ; 133.03 ; 134.06 ; 135.48 ; 138.05 ; 146.56$.

1-methyl-5-(1-(phenylsulfonyl)-1H-indol-3-yl)-1H-benzo[d][1,2,3]triazole was deprotected following general procedure $\mathrm{A}$ to afford 5-(1H-indol-3-yl)-1-methyl-1H-benzo[d][1,2,3]triazole (9). Purified by flash chromatography $\left(\mathrm{DCM} / \mathrm{MeOH}\right.$ 1:0 $\rightarrow$ 9: 1). ${ }^{1} \mathrm{H} \mathrm{NMR}\left(400 \mathrm{MHz}, \mathrm{CDCl}_{3}\right)$ : 国 $4.34(3 \mathrm{H}, \mathrm{s}), 7.24(1 \mathrm{H}, \mathrm{t}$, $\mathrm{J}=7.07 \mathrm{~Hz}), 7.29(1 \mathrm{H}, \mathrm{t}, \mathrm{J}=7.09 \mathrm{~Hz}), 7.45(1 \mathrm{H}, \mathrm{d}, \mathrm{J}=2.49 \mathrm{~Hz}), 7.48(1 \mathrm{H}, \mathrm{d}, \mathrm{J}=8.10 \mathrm{~Hz}), 7.58(1 \mathrm{H}, \mathrm{d}, \mathrm{J}=8.57$ $\mathrm{Hz}), 7.85(1 \mathrm{H}, \mathrm{dd}, \mathrm{J}=1.38,8.53 \mathrm{~Hz}), 7.98(1 \mathrm{H}, \mathrm{d}, \mathrm{J}=7.94 \mathrm{~Hz}), 8.31(1 \mathrm{H}, \mathrm{s}), 8.37(1 \mathrm{H}, \mathrm{s}) .{ }^{13} \mathrm{C} \mathrm{NMR}(100 \mathrm{MHz}$, $\left.\mathrm{CDCl}_{3}\right)$ : 目 34.31; 109.26; 111.54; 117.38; 117.73; 119.50; 120.63; 122.12; 122.69; 125.75; 128.33; 131.84; 132.36; 136.67; 146.91. HRMS: $\mathrm{m} / \mathrm{z}(\mathrm{El}+)$, calculated for : $\mathrm{C}_{15} \mathrm{H}_{12} \mathrm{~N}_{4} 249.1096$ [M] ${ }^{+}$, found: $249.1131[\mathrm{M}]^{+}$. Melting temp. range: $193-195^{\circ} \mathrm{C}$. 
<smiles>Cn1nnc2c([N+](=O)[O-])c(-c3c[nH]c4ccccc34)ccc21</smiles>

1-methyl-4-nitro-5-(1-(phenylsulfonyl)-1H-indol-3-yl)-1H-benzo[d][1,2,3]triazole was obtained after coupling $\mathbf{4 3}$ with $\mathbf{1 2 b}$. Purified by flash chromatography (DCM/MeOH 1:0 $\rightarrow 9: 1)$. Yield: $68 \%$. ${ }^{1 \mathrm{H}} \mathrm{NMR}$ $\left(400 \mathrm{MHz}, \mathrm{CDCl}_{3}\right)$ : 国 $4.45(3 \mathrm{H}, \mathrm{s}), 7.28(1 \mathrm{H}, \mathrm{t}, \mathrm{J}=7.53 \mathrm{~Hz}), 7.35-7.41(2 \mathrm{H}, \mathrm{m}), 7.42(1 \mathrm{H}, \mathrm{s}), 7.53(2 \mathrm{H}, \mathrm{t}$, $\mathrm{J}=7.58 \mathrm{~Hz}), 7.62(1 \mathrm{H}, \mathrm{t}, \mathrm{J}=7.35 \mathrm{~Hz}), 7.72(1 \mathrm{H}, \mathrm{d}, \mathrm{J}=8.57 \mathrm{~Hz}), 7.80(1 \mathrm{H}, \mathrm{s}), 7.87-7.96(3 \mathrm{H}, \mathrm{m}), 8.03(1 \mathrm{H}, \mathrm{d}$, $\mathrm{J}=8.30 \mathrm{~Hz}) .{ }^{13} \mathrm{C} \mathrm{NMR}\left(100 \mathrm{MHz}_{,} \mathrm{CDCl}_{3}\right)$ : 回 $34.93 ; 113.10 ; 113.75 ; 117.13 ; 119.41 ; 121.93 ; 124.08 ; 125.26$; $125.57 ; 126.72 ; 129.28 ; 129.48 ; 130.31 ; 134.26 ; 134.65 ; 137.42 ; 154.82$.

1-methyl-4-nitro-5-(1-(phenylsulfonyl)-1H-indol-3-yl)-1H-benzo[d][1,2,3]triazole was deprotected following general procedure $\mathrm{A}$ to afford 5-(1H-indol-3-yl)-1-methyl-4-nitro-1H-benzo[d][1,2,3]triazole (16). Purified by flash chromatography (DCM/MeOH 1:0 $\rightarrow$ 9: 1). Red powder. Yield: $46 \%$. $\underline{{ }^{1} \mathrm{H} N M R}$ (600MHz, DMSO): $4.44(3 \mathrm{H}, \mathrm{s}), 7.10(1 \mathrm{H}, \mathrm{t}, \mathrm{J}=7.48 \mathrm{~Hz}), 7.21(1 \mathrm{H}, \mathrm{t}, \mathrm{J}=7.55 \mathrm{~Hz}), 7.45(1 \mathrm{H}, \mathrm{d}, \mathrm{J}=7.98 \mathrm{~Hz})$, $7.51(1 \mathrm{H}, d, J=8.15 \mathrm{~Hz}), 7.61(1 \mathrm{H}, \mathrm{d}, J=2.50 \mathrm{~Hz}), 7.89(1 \mathrm{H}, \mathrm{d}, \mathrm{J}=8.63 \mathrm{~Hz}), 8.23(1 \mathrm{H}, \mathrm{d}, \mathrm{J}=8.62 \mathrm{~Hz}), 11.66$ $(1 \mathrm{H}, \mathrm{s}) .{ }^{13} \mathrm{C}$ NMR $(150 \mathrm{MHz}$, DMSO): 回 35.40; 109.75; 112.76; 115.22; 118.40; 120.61; 122.58; 124.89; $125.81 ; 126.03 ; 131.18 ; 134.12 ; 136.80 ; 137.58 ; 138.46$. HRMS: $\mathrm{m} / \mathrm{z}(\mathrm{El}+)$, calculated for: $\mathrm{C}_{15} \mathrm{H}_{13} \mathrm{~N}_{5}$ $294.0946[\mathrm{M}]^{+}$, found: $294.0987[\mathrm{M}]^{+}$. Melting temp. range: $269-277^{\circ} \mathrm{C}$.

- 5-(1H-indol-3-yl)-1-methyl-1H-benzo[d][1,2,3]triazol-4-amine (12)<smiles>Cn1nnc2c(N)c(-c3c[nH]c4ccccc34)ccc21</smiles>

1-methyl-5-(1-(phenylsulfonyl)-1H-indol-3-yl)-1H-benzo[d][1,2,3]triazol-4-amine was obtained after coupling 43 with 12c. Purified by flash chromatography (Cyclohexane/EtOAc 3:1 $\rightarrow 2: 1$ ). Yield: $65 \%$.

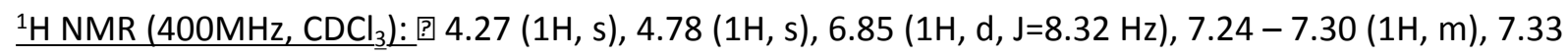
$(1 \mathrm{H}, \mathrm{d}, \mathrm{J}=8.32 \mathrm{~Hz}), 7.39(1 \mathrm{H}, \mathrm{t}, \mathrm{J}=7.56 \mathrm{~Hz}), 7.49(1 \mathrm{H}, \mathrm{t}, \mathrm{J}=7.50 \mathrm{~Hz}), 7.55-7.61(1 \mathrm{H}, \mathrm{m}), 7.71(1 \mathrm{H}, \mathrm{s}), 7.97$ $(1 \mathrm{H}, \mathrm{d}, \mathrm{J}=7.56 \mathrm{~Hz}), 8.10(1 \mathrm{H}, \mathrm{d}, \mathrm{J}=8.24 \mathrm{~Hz})$.

1-methyl-5-(1-(phenylsulfonyl)-1H-indol-3-yl)-1H-benzo[d][1,2,3]triazol-4-amine was deprotected following general procedure $A$ to afford 12. Purified by flash chromatography (Cyclohexane/EtOAc 4:1 $\rightarrow$ 1:1). Yield: $74 \%$. ${ }^{1} \mathrm{H} \mathrm{NMR}\left(400 \mathrm{MHz}_{1} \mathrm{CDCl}_{3}\right)$ : 国 $4.27(3 \mathrm{H}, \mathrm{s}), 4.85(2 \mathrm{H}, \mathrm{s}), 6.86(1 \mathrm{H}, \mathrm{d}, \mathrm{J}=8.30 \mathrm{~Hz}), 7.16$ $-7.19(1 \mathrm{H}, \mathrm{m}), 7.27-7.30(1 \mathrm{H}, \mathrm{m}), 7.37(2 \mathrm{H}, \mathrm{d}, \mathrm{J}=2.41 \mathrm{~Hz}), 7.45(1 \mathrm{H}, \mathrm{s}), 7.46(1 \mathrm{H}, \mathrm{s}), 7.49(2 \mathrm{H}, \mathrm{d}, \mathrm{J}=8.19$ $\mathrm{Hz}), 7.60(2 \mathrm{H}, \mathrm{d}, \mathrm{J}=7.57 \mathrm{~Hz}), 8.36(1 \mathrm{H}, \mathrm{br} \mathrm{s}) .{ }^{13} \mathrm{C} \mathrm{NMR}\left(100 \mathrm{MHz}, \mathrm{CDCl}_{3}\right)$ : ? 34.24; 96.85; 111.10; 111.44; $113.92 ; 120.11 ; 120.20 ; 122.65 ; 123.03 ; 126.58 ; 131.79 ; 134.10 ; 136.26 ; 136.61 ; 137.47$. HRMS: $\mathrm{m} / \mathrm{z}$ 
(El+), calculated for : $\mathrm{C}_{15} \mathrm{H}_{13} \mathrm{~N}_{5} 264.1205[\mathrm{M}]^{+}$, found: $264.1244[\mathrm{M}]^{+}$. Melting temp. range: $177-181$ ${ }^{\circ} \mathrm{C}$.

- 2-(6-(1H-indol-3-yl)-1H-benzo[d][1,2,3]triazol-1-yl)acetamide (21)<smiles>NC(=O)Cn1nnc2ccc(-c3c[nH]c4ccccc34)cc21</smiles>

2-(6-(1-(phenylsulfonyl)-1H-indol-3-yl)-1H-benzo[d][1,2,3]triazol-1-yl)acetamide was obtained after coupling $\mathbf{4 3}$ with $\mathbf{2 2 b}$. The coupling product was obtained bearing an acetamide instead of the initial acetonitrile. Purified by flash chromatography (cyclohexane/EtOAc 3:1). Yield: $80 \%$. ${ }^{1} \mathrm{H} \mathrm{NMR}(400 \mathrm{MHz}$, $\left.\mathrm{CDCl}_{3}\right)$ : $5.50(2 \mathrm{H}, \mathrm{s}), 7.39(1 \mathrm{H}, \mathrm{t}, \mathrm{J}=7.42 \mathrm{~Hz}), 7.44-7.50(2 \mathrm{H}, \mathrm{m}), 7.62(2 \mathrm{H}, \mathrm{t}, \mathrm{J}=7.56 \mathrm{~Hz}), 7.71(1 \mathrm{H}, \mathrm{t}$, $J=7.28 \mathrm{~Hz}), 7.80(1 \mathrm{H}, \mathrm{d}, \mathrm{J}=8.76 \mathrm{~Hz}), 7.88(1 \mathrm{H}, \mathrm{s}), 7.96(1 \mathrm{H}, \mathrm{d}, \mathrm{J}=7.92 \mathrm{~Hz}), 8.06(1 \mathrm{H}, \mathrm{d}, \mathrm{J}=8.24 \mathrm{~Hz}), 8.09-$ $8.17(4 \mathrm{H}, \mathrm{m}), 8.26(1 \mathrm{H}, \mathrm{s})$.

2-(6-(1-(phenylsulfonyl)-1H-indol-3-yl)-1H-benzo[d][1,2,3]triazol-1-yl)acetamide was deprotected following general procedure $\mathbf{B}$ to afford 22. Purified by flash chromatography (cyclohexane/EtOAc). Yield: $48 \%$. ${ }^{1} \mathrm{H}$ NMR $(600 \mathrm{MHz}, \mathrm{MeOD})$ : 回 $5.53(2 \mathrm{H}, \mathrm{s}), 7.17(1 \mathrm{H}, \mathrm{t}, \mathrm{J}=7.40 \mathrm{~Hz}), 7.21(1 \mathrm{H}, \mathrm{t}, \mathrm{J}=7.39 \mathrm{~Hz})$, $7.48(1 \mathrm{H}, \mathrm{d}, \mathrm{J}=8.02 \mathrm{~Hz}), 7.66(1 \mathrm{H}, \mathrm{s}), 7.83(1 \mathrm{H}, \mathrm{d}, \mathrm{J}=8.64 \mathrm{~Hz}), 7.94(1 \mathrm{H}, \mathrm{s}), 7.99(1 \mathrm{H}, \mathrm{d}, \mathrm{J}=7.88 \mathrm{~Hz}), 8.03$ $(1 \mathrm{H}, \mathrm{d}, \mathrm{J}=8.66 \mathrm{~Hz})$ Amide protons $\left(\mathrm{NH}_{2}\right)$ and indole $\mathrm{NH}$ not visible in $\mathrm{MeOD} .{ }^{13} \mathrm{C} \mathrm{NMR}(150 \mathrm{MHz}, \mathrm{MeOD})$ : ] 49.43; 106.30; 111.46; 115.90; 118.50; 118.73; 119.76; 121.67; 123.56; 125.02; 125.30; 134.72; 136.85; 137.43; 143.70; 169.28. HRMS: $\mathrm{m} / \mathrm{z}(\mathrm{El}+)$, calculated for: $\mathrm{C}_{16} \mathrm{H}_{14} \mathrm{~N}_{5} \mathrm{O} 292.1193$ [M] ${ }^{+}$, found: $292.1197[\mathrm{M}]^{+}$. Melting temp. range: $210-214^{\circ} \mathrm{C}$.

- 2-(6-(1H-indol-3-yl)-1H-benzo[d][1,2,3]triazol-1-yl)acetic acid (23)<smiles>O=C(O)Cn1nnc2ccc(-c3c[nH]c4ccccc34)cc21</smiles>

2-(6-(1-(phenylsulfonyl)-1H-indol-3-yl)-1H-benzo[d][1,2,3]triazol-1-yl)acetonitrile was deprotected following general procedure A. After completion, the reaction media was extracted with EtOAc and the $\mathrm{OL}$ was discarded. Then, the aqueous layer was acidified to $\mathrm{pH} 2$ and extracted again with EtOAc. The OL containing 24 was dried with $\mathrm{Na}_{2} \mathrm{SO}_{4}$ and evaporated. White powder. Yield: $72 \%$. $\underline{{ }^{1} \mathrm{H} \mathrm{NMR}}$ $\left(400 \mathrm{MHz}, \mathrm{CDCl}_{3}\right)$ : 回 $5.74(2 \mathrm{H}, \mathrm{s}), 7.13-7.25(2 \mathrm{H}, \mathrm{m}), 7.50(1 \mathrm{H}, \mathrm{d}, \mathrm{J}=7.87 \mathrm{~Hz}), 7.80(1 \mathrm{H}, \mathrm{d}, \mathrm{J}=8.73 \mathrm{~Hz})$, $7.88(1 \mathrm{H}, \mathrm{d}, \mathrm{J}=1.54 \mathrm{~Hz}), 8.04-8.13(3 \mathrm{H}, \mathrm{m}), 11.56(1 \mathrm{H}, \mathrm{s}) .{ }^{13} \mathrm{C} \mathrm{NMR}\left(100 \mathrm{MHz}, \mathrm{CDCl}_{3}\right):$ ? $49.10 ; 106.87$; $112.56 ; 115.53 ; 119.65 ; 119.77 ; 120.39 ; 122.22 ; 124.60 ; 125.27 ; 125.30 ; 134.95 ; 135.96 ; 137.49$; 143.92; 169.43. MS: $\mathrm{m} / \mathrm{z}(\mathrm{El}+)$, calculated for $\mathrm{C}_{16} \mathrm{H}_{13} \mathrm{~N}_{4} \mathrm{O}_{2}^{+}$: [M] ${ }^{+}$293.1, found: $293.1[\mathrm{M}]^{+}$. Melting temp. range: $248-255^{\circ} \mathrm{C}$.

- 2-(5-(1H-indol-3-yl)-1H-benzo[d][1,2,3]triazol-1-yl)acetamide (22) 
<smiles>NC(=O)Cn1nnc2cc(-c3c[nH]c4ccccc34)ccc21</smiles>

2-(5-(1-(phenylsulfonyl)-1H-indol-3-yl)-1H-benzo[d][1,2,3]triazol-1-yl)acetamide was obtained after coupling $\mathbf{4 3}$ with $\mathbf{2 2 b}$. The coupling product was obtained bearing an acetamide instead of the initial acetonitrile. Purified by flash chromatography (cyclohexane/EtOAc 3:1). Yield: $83 \%$. ${ }^{1} \mathrm{H} \mathrm{NMR}(400 \mathrm{MHz}$, $\left.\mathrm{CDCl}_{3}\right)$ : $5.48(2 \mathrm{H}, \mathrm{s}), 7.38(1 \mathrm{H}, \mathrm{t}, \mathrm{J}=7.47 \mathrm{~Hz}), 7.46(2 \mathrm{H}, \mathrm{t}, \mathrm{J}=7.79 \mathrm{~Hz}), 7.50(1 \mathrm{H}, \mathrm{s}), 7.63(2 \mathrm{H}, \mathrm{t}, \mathrm{J}=7.55 \mathrm{~Hz})$, $7.72(1 \mathrm{H}, \mathrm{t}, \mathrm{J}=7.24 \mathrm{~Hz}), 7.92(4 \mathrm{H}, \mathrm{d}, \mathrm{J}=7.19 \mathrm{~Hz}), 8.07(1 \mathrm{H}, \mathrm{d}, \mathrm{J}=8.16 \mathrm{~Hz}), 8.13(3 \mathrm{H}, \mathrm{d}, \mathrm{J}=7.68 \mathrm{~Hz}), 8.25(1 \mathrm{H}$, s), $8.37(1 \mathrm{H}, \mathrm{s})$.

2-(5-(1-(phenylsulfonyl)-1H-indol-3-yl)-1H-benzo[d][1,2,3]triazol-1-yl)acetamide was deprotected following general procedure $B$ to afford 23. Purified by flash chromatography (cyclohexane/EtOAc). Yield: 49 \%. ${ }^{1} \mathrm{H}$ NMR $(400 \mathrm{MHz}$, DMSO): $5.44(2 \mathrm{H}, \mathrm{s}), 7.14(1 \mathrm{H}, \mathrm{t}, \mathrm{J}=7.32 \mathrm{~Hz}), 7.19(1 \mathrm{H}, \mathrm{t}, \mathrm{J}=7.33 \mathrm{~Hz})$, $7.44-7.54(2 \mathrm{H}, \mathrm{m}), 7.78-7.85(2 \mathrm{H}, \mathrm{m}), 7.85-7.99(3 \mathrm{H}, \mathrm{m}), 8.23(1 \mathrm{H}, \mathrm{s}), 11.45(1 \mathrm{H}, \mathrm{s}) .{ }^{13} \mathrm{C} \mathrm{NMR}$ (100MHz, DMSO): 0 0.22; 111.67; 112.51; 115.31; 115.70; 119.31; 120.27; 122.04; 124.47; 125.47; 128.02; 137.38; 146.69; 168.02. HRMS: $\mathrm{m} / \mathrm{z}(\mathrm{El}+)$, calculated for: $\mathrm{C}_{16} \mathrm{H}_{14} \mathrm{~N}_{5} \mathrm{O} 292.1193$ [M] ${ }^{+}$, found: $292.1195[\mathrm{M}]^{+}$. Melting temp. range: $252-254^{\circ} \mathrm{C}$.

- 2-(5-(1H-indol-3-yl)-1H-benzo[d][1,2,3]triazol-1-yl)acetic acid (24)<smiles>O=C(O)Cn1nnc2cc(-c3c[nH]c4ccccc34)ccc21</smiles>

2-(5-(1-(phenylsulfonyl)-1H-indol-3-yl)-1H-benzo[d][1,2,3]triazol-1-yl)acetonitrile was deprotected following general procedure A. After completion, the reaction media was extracted with EtOAc and the $\mathrm{OL}$ was discarded. Then, the aqueous layer was acidified to $\mathrm{pH} 2$ and extracted again with EtOAc. The OL containing 25 was dried with $\mathrm{Na}_{2} \mathrm{SO}_{4}$ and evaporated. White powder. Yield: $63 \%$. ${ }^{1} \mathrm{H} \mathrm{NMR}$ $\left(400 \mathrm{MHz}, \mathrm{CDCl}_{3}\right)$ : 国 $5.69(2 \mathrm{H}, \mathrm{s}), 7.12-7.22(2 \mathrm{H}, \mathrm{m}), 7.49(1 \mathrm{H}, \mathrm{d}, \mathrm{J}=7.86 \mathrm{~Hz}), 7.83(1 \mathrm{H}, \mathrm{d}, \mathrm{J}=2.31 \mathrm{~Hz})$, $7.89-7.98(3 \mathrm{H}, \mathrm{m}), 8.25(1 \mathrm{H}, \mathrm{s}), 11.47(1 \mathrm{H}, \mathrm{s}), 13.46(1 \mathrm{H}, \mathrm{br} \mathrm{s}) .{ }^{13} \mathrm{C} \mathrm{NMR}\left(100 \mathrm{MHz}, \mathrm{CDCl}_{3}\right)$ : [ $111.54 ; 112.51 ; 115.36 ; 115.62 ; 119.32 ; 120.29 ; 122.05 ; 124.54 ; 125.45 ; 128.22 ; 132.51 ; 132.59$; 137.38; 146.55; 169.27. MS: $\mathrm{m} / \mathrm{z}(\mathrm{El}+)$, calculated for $\mathrm{C}_{16} \mathrm{H}_{13} \mathrm{~N}_{4} \mathrm{O}_{2}{ }^{+}: 293.1[\mathrm{M}]^{+}$, found: $293.1[\mathrm{M}]^{+}$. Melting temp. range: $250-252^{\circ} \mathrm{C}$.

2.12.2 List of compounds obtained following cross-coupling general procedure B (using 41)

A. Using 1-(phenylsulfonyl)-3-(4,4,5,5-tetramethyl-1,3,2-dioxaborolan-2-yl)-1H-indole

- 6-(1H-indol-3-yl)-2-methyl-2H-[1,2,3]triazolo[4,5-b]pyridine (13) 
<smiles>Cn1nc2cc(-c3c[nH]c4ccccc34)cnc2n1</smiles>

2-methyl-6-(1-(phenylsulfonyl)-1H-indol-3-yl)-2H-[1,2,3]triazolo[4,5-b]pyridine

was obtained after coupling 41 with 13. Purified by flash chromatography (Cyclohexane/EtOAc $4: 1 \rightarrow 1: 1)$. Yield: $77 \%$. ${ }^{1 \mathrm{H} N M R}\left(400 \mathrm{MHz} \mathrm{CDCl}_{3}\right)$ : ? $4.40(3 \mathrm{H}, \mathrm{s}), 7.37(1 \mathrm{H}, \mathrm{t}, \mathrm{J}=7.57 \mathrm{~Hz})$, $7.46(1 \mathrm{H}, \mathrm{t}, \mathrm{J}=8.05 \mathrm{~Hz}), 7.51(2 \mathrm{H}, \mathrm{t}, \mathrm{J}=7.70 \mathrm{~Hz}), 7.60(1 \mathrm{H}, \mathrm{t}, \mathrm{J}=7.47 \mathrm{~Hz}), 7.76(1 \mathrm{H}, \mathrm{d}, \mathrm{J}=7.93$ $\mathrm{Hz}), 7.87(1 \mathrm{H}, \mathrm{s}), 7.98(2 \mathrm{H}, \mathrm{d}, \mathrm{J}=7.51 \mathrm{~Hz}), 8.07(1 \mathrm{H}, \mathrm{d}, J=1.78 \mathrm{~Hz}), 8.12(1 \mathrm{H}, \mathrm{d}, J=8.32 \mathrm{~Hz})$, $9.01(1 \mathrm{H}, \mathrm{d}, \mathrm{J}=1.80 \mathrm{~Hz})$.

2-methyl-6-(1-(phenylsulfonyl)-1H-indol-3-yl)-2H-[1,2,3]triazolo[4,5-b]pyridine

was deprotected following general procedure $A$ to afford 13. Purified by flash chromatography (Cyclohexane/EtOAc 3:1 $\rightarrow$ 1:1). Yield: $68 \%$ 새 NMR (400MHz, DMSO): ? $4.40(3 \mathrm{H}, \mathrm{s}), 7.19$ $(1 \mathrm{H}, \mathrm{t}, \mathrm{J}=6.98 \mathrm{~Hz}), 7.23(1 \mathrm{H}, \mathrm{t}, \mathrm{J}=7.02 \mathrm{~Hz}), 7.52(1 \mathrm{H}, \mathrm{d}, \mathrm{J}=7.76 \mathrm{~Hz}), 8.05(1 \mathrm{H}, \mathrm{s}), 8.11(1 \mathrm{H}, \mathrm{d}$, $\mathrm{J}=7.75 \mathrm{~Hz}), 8.33(1 \mathrm{H}, \mathrm{s}), 8.54(1 \mathrm{H}, \mathrm{d}, \mathrm{J}=1.88 \mathrm{~Hz}), 9.11(1 \mathrm{H}, \mathrm{d}, \mathrm{J}=1.90 \mathrm{~Hz}), 11.75(1 \mathrm{H}, \mathrm{s}) .{ }^{13} \mathrm{C}$ NMR (100MHz, DMSO): ? 35.28; 112.30; 112.70; 114.82; 119.62; 120.69; 122.49; 125.23; $125.91 ; 126.86 ; 131.05 ; 137.45 ; 148.39 ; 155.53$. $\underline{\text { HRMS: }} \mathrm{m} / \mathrm{z}(\mathrm{EI}+)$, calculated for: $\mathrm{C}_{14} \mathrm{H}_{11} \mathrm{~N}_{5}$ $250.1048[\mathrm{M}]^{+}$, found: $250.1086[\mathrm{M}]^{+}$. Melting temp. range: $257-260{ }^{\circ} \mathrm{C}$.

- 6-(1H-indol-3-yl)-1-methyl-1H-[1,2,3]triazolo[4,5-b]pyridine (14)<smiles>Cn1nnc2ncc(-c3c[nH]c4ccccc34)cc21</smiles>

1-methyl-6-(1-(phenylsulfonyl)-1H-indol-3-yl)-1H-[1,2,3]triazolo[4,5-b]pyridine was obtained after coupling 41 with 14 . Purified by flash chromatography (Cyclohexane/EtOAc $4: 1 \rightarrow 1: 1)$. Yield: $49 \%$. ${ }^{1 \mathrm{H} \mathrm{NMR}}\left(400 \mathrm{MHz}_{1} \mathrm{CDCl}_{3}\right)$ : 国 $4.60(3 \mathrm{H}, \mathrm{s}), 7.36(1 \mathrm{H}, \mathrm{t}, \mathrm{J}=7.63 \mathrm{~Hz})$, $7.44(2 \mathrm{H}, \mathrm{t}, \mathrm{J}=7.71 \mathrm{~Hz}), 7.50(3 \mathrm{H}, \mathrm{t}, \mathrm{J}=7.71 \mathrm{~Hz}), 7.59(1 \mathrm{H}, \mathrm{t}, \mathrm{J}=7.48 \mathrm{~Hz}), 7.78(1 \mathrm{H}, \mathrm{d}, \mathrm{J}=7.82$ $\mathrm{Hz}), 7.85(1 \mathrm{H}, \mathrm{s}), 7.98(2 \mathrm{H}, \mathrm{d}, \mathrm{J}=7.54 \mathrm{~Hz}), 8.11(1 \mathrm{H}, \mathrm{d}, \mathrm{J}=8.30 \mathrm{~Hz}), 8.39(1 \mathrm{H}, \mathrm{d}, J=1.97 \mathrm{~Hz})$, $9.04(1 \mathrm{H}, \mathrm{d}, \mathrm{J}=1.93 \mathrm{~Hz})$.

1-methyl-6-(1-(phenylsulfonyl)-1H-indol-3-yl)-1H-[1,2,3]triazolo[4,5-b]pyridine

was deprotected following general procedure $A$ to afford 14. Purified by flash chromatography (Cyclohexane/EtOAc 3:1 $\rightarrow$ 1:1). ${ }^{1} \mathrm{H}$ NMR $(400 \mathrm{MHz}, \mathrm{DMSO})$ : ? $4.55(3 \mathrm{H}, \mathrm{s}), 7.17(1 \mathrm{H}, \mathrm{t}$, $\mathrm{J}=7.34 \mathrm{~Hz}), 7.22(1 \mathrm{H}, \mathrm{t}, \mathrm{J}=7.13 \mathrm{~Hz}), 7.51(1 \mathrm{H}, \mathrm{d}, \mathrm{J}=7.96 \mathrm{~Hz}), 7.98(1 \mathrm{H}, \mathrm{d}, \mathrm{J}=7.81 \mathrm{~Hz}), 8.02(1 \mathrm{H}$, d, J=2.52 Hz), $8.54(1 \mathrm{H}, \mathrm{d}, \mathrm{J}=1.99 \mathrm{~Hz}), 9.19(1 \mathrm{H}, \mathrm{d}, \mathrm{J}=1.91 \mathrm{~Hz}), 11.64(1 \mathrm{H}, \mathrm{s}) . \underline{{ }^{13} \mathrm{C} \mathrm{NMR}}$ (150MHz, DMSO): $44.26 ; 112.63 ; 112.66 ; 119.36 ; 120.72 ; 120.86 ; 122.44 ; 125.31$; 


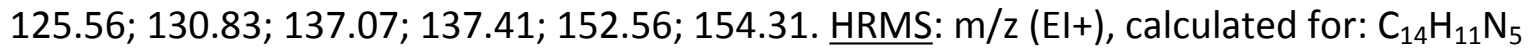
$250.1048[\mathrm{M}]^{+}$, found: $250.1086[\mathrm{M}]^{+}$. Melting temp. range: $206-211^{\circ} \mathrm{C}$.

- 6-(1H-indol-3-yl)-3-methyl-3H-[1,2,3]triazolo[4,5-b]pyridine (15)<smiles>Cn1nnc2cc(-c3c[nH]c4ccccc34)cnc21</smiles>

3-methyl-6-(1-(phenylsulfonyl)-1H-indol-3-yl)-3H-[1,2,3]triazolo[4,5-b]pyridine pyridine was obtained after coupling 41 with 14 . Purified by flash chromatography

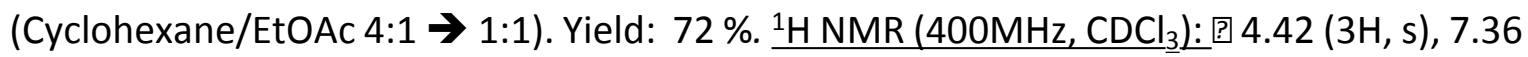
$(1 \mathrm{H}, \mathrm{t}, \mathrm{J}=7.32 \mathrm{~Hz}), 7.44(1 \mathrm{H}, \mathrm{t}, \mathrm{J}=7.72 \mathrm{~Hz}), 7.50(2 \mathrm{H}, \mathrm{t}, \mathrm{J}=7.69 \mathrm{~Hz}), 7.59(1 \mathrm{H}, \mathrm{t}, \mathrm{J}=7.46 \mathrm{~Hz})$, $7.74(1 \mathrm{H}, \mathrm{d}, \mathrm{J}=7.87 \mathrm{~Hz}), 7.83(1 \mathrm{H}, \mathrm{s}), 7.98(2 \mathrm{H}, \mathrm{d}, \mathrm{J}=7.45 \mathrm{~Hz}), 8.12(1 \mathrm{H}, \mathrm{d}, \mathrm{J}=8.35 \mathrm{~Hz}), 8.55$ $(1 \mathrm{H}, \mathrm{d}, \mathrm{J}=1.77 \mathrm{~Hz}), 8.92(1 \mathrm{H}, \mathrm{d}, \mathrm{J}=1.75 \mathrm{~Hz})$.

3-methyl-6-(1-(phenylsulfonyl)-1H-indol-3-yl)-3H-[1,2,3]triazolo[4,5-b]pyridine was deprotected following general procedure $A$ to afford 15. Purified by flash chromatography (Cyclohexane/EtOAc 3:1 $\rightarrow$ 1:1). Yield: $75 \%$. ${ }^{1} \mathrm{H} \mathrm{NMR}(400 \mathrm{MHz}, \mathrm{DMSO})$ : 囯 $4.33(3 \mathrm{H}, \mathrm{s}), 7.16$ $(1 \mathrm{H}, \mathrm{t}, \mathrm{J}=7.39 \mathrm{~Hz}), 7.22(1 \mathrm{H}, \mathrm{t}, \mathrm{J}=7.45 \mathrm{~Hz}), 7.51(1 \mathrm{H}, \mathrm{d}, \mathrm{J}=7.88 \mathrm{~Hz}), 7.94(1 \mathrm{H}, \mathrm{t}, \mathrm{J}=3.19 \mathrm{~Hz})$, $8.68(1 \mathrm{H}, \mathrm{d}, \mathrm{J}=1.03 \mathrm{~Hz}), 9.13(1 \mathrm{H}, \mathrm{d}, \mathrm{J}=1.02 \mathrm{~Hz}), 11.59(1 \mathrm{H}, \mathrm{s}) .{ }^{13} \mathrm{C}$ NMR $(100 \mathrm{MHz}, \mathrm{DMSO})$ : ? $33.35 ; 112.30 ; 112.62 ; 119.10 ; 120.67 ; 122.39 ; 123.88 ; 125.21 ; 125.30 ; 129.12 ; 137.25$; 137.31; 144.49; 150.71. HRMS: $\mathrm{m} / \mathrm{z}(\mathrm{El}+)$, calculated for: $\mathrm{C}_{14} \mathrm{H}_{11} \mathrm{~N}_{5} 250.1048$ [M] ${ }^{+}$, found: $250.1089[\mathrm{M}]^{+}$. Melting temp. range: $207-209^{\circ} \mathrm{C}$.

- 6-(1H-indol-3-yl)isoquinoline (2)<smiles>c1ccc2c(-c3ccc4cnccc4c3)c[nH]c2c1</smiles>

6-(1-(phenylsulfonyl)-1H-indol-3-yl)isoquinoline was obtained after coupling $\mathbf{4 1}$ with 6bromoisoquinoline. Purified by flash chromatography (Cyclohexane/EtOAc 3:1 $\rightarrow 0: 1$ ). Yield: \%. $\underline{{ }^{1} \mathrm{H}}$ NMR $\left(400 \mathrm{MHz}_{1} \mathrm{CDCl}_{3}\right)$ : 国 $7.36(1 \mathrm{H}, \mathrm{t}, \mathrm{J}=7.48 \mathrm{~Hz}), 7.43(1 \mathrm{H}, \mathrm{t}, \mathrm{J}=7.64 \mathrm{~Hz}), 7.48(2 \mathrm{H}, \mathrm{t}, \mathrm{J}=7.72 \mathrm{~Hz}), 7.57(1 \mathrm{H}$, $\mathrm{t}, \mathrm{J}=7.40 \mathrm{~Hz}), 7.70(1 \mathrm{H}, \mathrm{d}, \mathrm{J}=5.72 \mathrm{~Hz}), 7.84-7.90(3 \mathrm{H}, \mathrm{m}), 7.97(2 \mathrm{H}, \mathrm{d}, \mathrm{J}=7.60 \mathrm{~Hz}), 8.04-8.14(3 \mathrm{H}, \mathrm{m})$, $8.56(1 \mathrm{H}, \mathrm{d}, \mathrm{J}=5.72 \mathrm{~Hz}), 9.28(1 \mathrm{H}, \mathrm{s}) .{ }^{13} \mathrm{C}$ NMR $\left(100 \mathrm{MHz}, \mathrm{CDCl}_{3}\right)$ : 回 113.98; 120.36; 120.52; 123.16; $124.00 ; 124.04 ; 124.75 ; 125.40 ; 126.93 ; 127.60 ; 127.79 ; 128.42 ; 128.87 ; 129.47 ; 134.13 ; 135.29$; $135.57 ; 136.20 ; 138.03 ; 143.50 ; 152.24$. 
6-(1-(phenylsulfonyl)-1H-indol-3-yl)isoquinoline was deprotected following general procedure $\mathrm{A}$ to

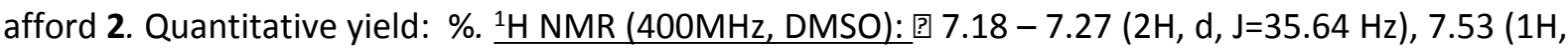
d, J=7.49 Hz), $7.90(1 \mathrm{H}, \mathrm{d}, \mathrm{J}=5.40 \mathrm{~Hz}), 8.03(1 \mathrm{H}, \mathrm{s}), 8.08-8.17(3 \mathrm{H}, \mathrm{m}), 8.29(1 \mathrm{H}, \mathrm{s}), 8.48(1 \mathrm{H}, \mathrm{d}, \mathrm{J}=5.55$ $\mathrm{Hz}), 9.26(1 \mathrm{H}, \mathrm{s}), 11.66(1 \mathrm{H}, \mathrm{s}) .{ }^{13} \mathrm{C}$ NMR $(100 \mathrm{MHz}, \mathrm{DMSO})$ : $112.69 ; 115.20 ; 119.88 ; 120.66 ; 120.71$; $121.66 ; 122.34 ; 125.27 ; 126.01 ; 126.91 ; 127.62 ; 128.36 ; 136.63 ; 137.66 ; 138.54 ; 143.60 ; 152.20$. HRMS: $\mathrm{m} / \mathrm{z}(\mathrm{El}+)$, calculated for : $\mathrm{C}_{17} \mathrm{H}_{11} \mathrm{~N}_{2} 245.1034[\mathrm{M}]^{+}$, found: 245.1073 [M] ${ }^{+}$. Melting temp. range: $219-223^{\circ} \mathrm{C}$.

- 7-(1H-indol-3-yl)isoquinoline (1)<smiles>c1ccc2c(-c3ccc4ccncc4c3)c[nH]c2c1</smiles>

7-(1-(phenylsulfonyl)-1H-indol-3-yl)isoquinoline was obtained after coupling $\mathbf{4 1}$ with 5bromoisoquinoline. Purified by flash chromatography (Cyclohexane/EtOAc $4: 1 \rightarrow 1: 4)$. Yield: \%. $\underline{{ }^{1} \mathrm{H}}$ NMR $\left(400 \mathrm{MHz}, C D C l_{3}\right)$ : 国 $7.36(1 \mathrm{H}, \mathrm{t}, \mathrm{J}=7.50 \mathrm{~Hz}), 7.43(1 \mathrm{H}, \mathrm{t}, \mathrm{J}=7.58 \mathrm{~Hz}), 7.48(2 \mathrm{H}, \mathrm{t}, \mathrm{J}=7.72 \mathrm{~Hz}), 7.57(1 \mathrm{H}$, t, J=7.42 Hz), $7.70(1 \mathrm{H}, \mathrm{d}, \mathrm{J}=5.72 \mathrm{~Hz}), 7.84-7.89(2 \mathrm{H}, \mathrm{m}), 7.92-8.00(4 \mathrm{H}, \mathrm{m}), 8.11(1 \mathrm{H}, \mathrm{d}, \mathrm{J}=8.24 \mathrm{~Hz})$, $8.20(1 \mathrm{H}, \mathrm{s}), 8.55(1 \mathrm{H}, \mathrm{d}, \mathrm{J}=5.72 \mathrm{~Hz}), 9.31(1 \mathrm{H}, \mathrm{s}) .{ }^{13} \mathrm{C} \mathrm{NMR}\left(100 \mathrm{MHz}_{1} \mathrm{CDCl}_{3}\right):$ ? $113.98 ; 120.29 ; 120.40$; 123.18 ; 123.63; 123.97; 125.36; 125.93; 126.91; 127.30; 128.96; 128.99; 129.45; 130.69; 132.18; $134.09 ; 135.05 ; 135.55 ; 138.05 ; 143.10 ; 152.48$.

7-(1-(phenylsulfonyl)-1H-indol-3-yl)isoquinoline was deprotected following general procedure $A$ to afford 1. Quantitative yield. ${ }^{1} \mathrm{H} N M R(400 \mathrm{MHz}, \mathrm{DMSO})$ : ? $7.22(2 \mathrm{H}, \mathrm{m}), 7.52(1 \mathrm{H}, \mathrm{d}, \mathrm{J}=7.44 \mathrm{~Hz}), 7.82$ $(1 \mathrm{H}, \mathrm{d}, \mathrm{J}=5.13 \mathrm{~Hz}), 7.97-8.05(2 \mathrm{H}, \mathrm{t}, \mathrm{J}=9.61 \mathrm{~Hz}), 8.14(1 \mathrm{H}, \mathrm{d}, \mathrm{J}=7.30 \mathrm{~Hz}), 8.20(1 \mathrm{H}, \mathrm{d}, \mathrm{J}=8.23 \mathrm{~Hz}), 8.46$ $(2 \mathrm{H}, \mathrm{d}, \mathrm{J}=8.28 \mathrm{~Hz}), 9.41(1 \mathrm{H}, \mathrm{s}) .{ }^{13} \mathrm{C}$ NMR $(100 \mathrm{MHz}, \mathrm{DMSO})$ : 112.62; 115.29; 119.80; 120.48; 120.56; $122.24 ; 122.97 ; 125.27 ; 125.30 ; 127.29 ; 129.59 ; 130.78 ; 133.75 ; 135.53 ; 137.58 ; 142.47 ; 152.65$. HRMS: $\mathrm{m} / \mathrm{z}(\mathrm{El}+)$, calculated for: $\mathrm{C}_{17} \mathrm{H}_{11} \mathrm{~N}_{2} 245.1034$ [M] ${ }^{+}$, found: $245.1073[\mathrm{M}]^{+}$. Melting temp. range: $208-214^{\circ} \mathrm{C}$.

- 7-(1H-indol-3-yl)quinazoline (4)<smiles>c1ccc2c(-c3ccc4cncnc4c3)c[nH]c2c1</smiles>

7-(1-(phenylsulfonyl)-1H-indol-3-yl)quinazoline was obtained after coupling $\mathbf{4 1}$ with 7bromoquinazoline. Purified by flash chromatography (Cyclohexane/EtOAc $4: 1 \rightarrow 1: 4)$. Yellow powder. Yield: $56 \%$. ${ }^{1} \mathrm{H}$ NMR $\left(400 \mathrm{MHz}, \mathrm{CDCl}_{3}\right)$ : 回 $7.37(1 \mathrm{H}, \mathrm{t}, \mathrm{J}=7.48 \mathrm{~Hz}), 7.44(1 \mathrm{H}, \mathrm{t}, \mathrm{J}=7.66 \mathrm{~Hz}), 7.49(2 \mathrm{H}, \mathrm{t}, \mathrm{J}=7.72$ $\mathrm{Hz}), 7.59(1 \mathrm{H}, \mathrm{t}, \mathrm{J}=7.40 \mathrm{~Hz}), 7.94-7.99(5 \mathrm{H}, \mathrm{m}), 8.04(1 \mathrm{H}, \mathrm{d}, \mathrm{J}=8.40 \mathrm{~Hz}), 8.11(1 \mathrm{H}, \mathrm{d}, \mathrm{J}=8.28 \mathrm{~Hz}), 8.32$ $(1 \mathrm{H}, \mathrm{s}), 9.36(1 \mathrm{H}, \mathrm{s}), 9.43(1 \mathrm{H}, \mathrm{s})$. 
7-(1-(phenylsulfonyl)-1H-indol-3-yl)quinazoline was deprotected following general procedure $\mathrm{A}$ to afford 4. Quantitative yield. ${ }^{1} \mathrm{H}$ NMR $(400 \mathrm{MHz}, \mathrm{DMSO})$ : ] $7.18-7.27(2 \mathrm{H}, \mathrm{m}), 7.53(1 \mathrm{H}, \mathrm{d}, \mathrm{J}=7.07 \mathrm{~Hz})$, $8.08(1 \mathrm{H}, \mathrm{d}, \mathrm{J}=7.76 \mathrm{~Hz}), 8.12-8.24(3 \mathrm{H}, \mathrm{q}, \mathrm{J}=9.53 \mathrm{~Hz}), 8.26(1 \mathrm{H}, \mathrm{s}), 9.25(1 \mathrm{H}, \mathrm{s}), 9.51(1 \mathrm{H}, \mathrm{s}), 11.79(1 \mathrm{H}$,

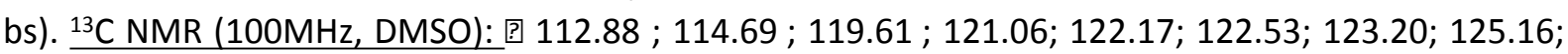
$127.05 ; 128.05 ; 128.47 ; 137.77 ; 142.80 ; 150.80 ; 155.93 ; 160.04$. HRMS: $\mathrm{m} / \mathrm{z}$ (El+), calculated for: $\mathrm{C}_{16} \mathrm{H}_{11} \mathrm{~N}_{3} 246.0987[\mathrm{M}]^{+}$, found: $246.1102[\mathrm{M}]^{+}$. Melting temp. range: $221-226^{\circ} \mathrm{C}$.

- 6-(1H-indol-3-yl)quinazoline (3)<smiles>c1ccc2c(-c3ccc4ncncc4c3)c[nH]c2c1</smiles>

6-(1-(phenylsulfonyl)-1H-indol-3-yl)quinazoline was obtained after coupling $\mathbf{4 1}$ with 6bromoquinazoline. Purified by flash chromatography (Cyclohexane/EtOAc 4:1 $\rightarrow$ 1:4). Yield: $68 \%$. $\underline{{ }^{1} \mathrm{H}}$ $\operatorname{NMR}\left(400 \mathrm{MHz}, C D \mathrm{Cl}_{3}\right)$ : 回 $7.37(1 \mathrm{H}, \mathrm{t}, \mathrm{J}=7.34 \mathrm{~Hz}), 7.44(1 \mathrm{H}, \mathrm{t}, \mathrm{J}=7.50 \mathrm{~Hz}), 7.49(2 \mathrm{H}, \mathrm{t}, \mathrm{J}=7.72 \mathrm{~Hz}), 7.59(1 \mathrm{H}$, t, J=7.43 Hz), $7.85(1 \mathrm{H}, \mathrm{d}, \mathrm{J}=7.95 \mathrm{~Hz}), 7.88(1 \mathrm{H}, \mathrm{s}), 7.98(1 \mathrm{H}, \mathrm{d}, \mathrm{J}=7.50 \mathrm{~Hz}), 8.12(1 \mathrm{H}, \mathrm{d}, \mathrm{J}=8.27 \mathrm{~Hz}), 8.14$ $-8.22(4 \mathrm{H}, \mathrm{m}), 9.36(1 \mathrm{H}, \mathrm{s}), 9.47(1 \mathrm{H}, \mathrm{s})$.

6-(1-(phenylsulfonyl)-1H-indol-3-yl)quinazoline was deprotected following general procedure $\mathrm{A}$ to afford 3. Quantitative yield. ${ }^{1} \mathrm{H} N M R(400 \mathrm{MHz}, \mathrm{DMSO})$ : ? $7.16-7.26(2 \mathrm{H}, \mathrm{m}), 7.53(1 \mathrm{H}, \mathrm{d}, \mathrm{J}=7.56 \mathrm{~Hz})$, $7.99-8.07(2 \mathrm{H}, \mathrm{d}, \mathrm{J}=8.00 \mathrm{~Hz}), 8.15(1 \mathrm{H}, \mathrm{d}, \mathrm{J}=7.52 \mathrm{~Hz}), 8.41-8.50(2 \mathrm{H}, \mathrm{m}), 9.22(1 \mathrm{H}, \mathrm{s}), 9.68(2 \mathrm{H}, \mathrm{s})$, $11.84(1 \mathrm{H}, \mathrm{s}) .{ }_{13}^{13} \mathrm{C} \mathrm{NMR}(100 \mathrm{MHz}, \mathrm{DMSO}):$ ? $112.76 ; 114.54 ; 119.75 ; 120.61 ; 122.33 ; 122.42 ; 125.16$; $125.85 ; 126.05 ; 128.41 ; 134.66 ; 136.18 ; 137.66 ; 148.19 ; 154.54 ; 160.67$. HRMS: $\mathrm{m} / \mathrm{z}(\mathrm{El}+)$, calculated for : $\mathrm{C}_{16} \mathrm{H}_{11} \mathrm{~N}_{3} 246.0987[\mathrm{M}]^{+}$, found: $246.1102[\mathrm{M}]^{+}$. Melting temp. range: $271-275^{\circ} \mathrm{C}$.

- 6-(1H-indol-3-yl)-3,4-dihydroquinazolin-2(1H)-one (5)<smiles>O=C1NCc2cc(-c3c[nH]c4ccccc34)ccc2N1</smiles>

6-(1-(phenylsulfonyl)-1H-indol-3-yl)-3,4-dihydroquinazolin-2(1H)-one was obtained after coupling 41 with 6-bromo-3,4-dihydroquinazolin-2(1H)-one. Purified by flash chromatography

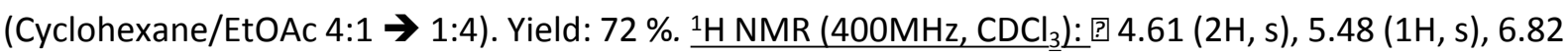
$(1 \mathrm{H}, \mathrm{d}, \mathrm{J}=8.14 \mathrm{~Hz}), 7.32(1 \mathrm{H}, \mathrm{s}), 7.24-7.32(2 \mathrm{H}, \mathrm{m}), 7.35-7.49(4 \mathrm{H}, \mathrm{m}), 7.52-7.58(1 \mathrm{H}, \mathrm{m}), 7.64(1 \mathrm{H}$, s), $7.71(1 \mathrm{H}, \mathrm{d}, J=7.80 \mathrm{~Hz}), 7.83(1 \mathrm{H}, \mathrm{s}), 7.92(2 \mathrm{H}, \mathrm{d}, \mathrm{J}=7.53 \mathrm{~Hz}), 8.06(1 \mathrm{H}, \mathrm{d}, J=8.28 \mathrm{~Hz})$.

6-(1-(phenylsulfonyl)-1H-indol-3-yl)-3,4-dihydroquinazolin-2(1H)-one was deprotected following general procedure A to afford 5. Quantitative yield. ${ }^{1} \mathrm{H} \mathrm{NMR}(400 \mathrm{MHz}, \mathrm{DMSO}):$ ? $4.39(2 \mathrm{H}, \mathrm{s}), 6.84(1 \mathrm{H}$, d, J=7.96 Hz), $7.10(1 \mathrm{H}, \mathrm{td}, \mathrm{J}=7.19,27.43 \mathrm{~Hz}), 7.38-7.46(3 \mathrm{H}, \mathrm{m}), 7.56(1 \mathrm{H}, \mathrm{s}), 7.84(1 \mathrm{H}, \mathrm{d}, \mathrm{J}=7.69 \mathrm{~Hz})$, $9.02(1 \mathrm{H}, \mathrm{br} \mathrm{s}), 11.25$ (1H, br s). ${ }^{13} \mathrm{C}$ NMR (150MHz, DMSO): ? $43.17 ; 112.30 ; 114.43 ; 116.04 ; 119.02$; 
$119.57 ; 119.79 ; 121.74 ; 122.97 ; 124.28 ; 125.45 ; 126.32 ; 129.28 ; 136.23 ; 137.24 ; 155.09$. HRMS: $\mathrm{m} / \mathrm{z}$ (El+), calculated for : $286.0912[\mathrm{M}+\mathrm{Na}]^{+}$, found: $286.1060[\mathrm{M}+\mathrm{Na}]^{+}$. Melting temp. range: $256-265$ ${ }^{\circ} \mathrm{C}$.

- 1-(cyclopropylmethyl)-6-(1H-indol-3-yl)-1H-benzo[d][1,2,3]triazole (19)<smiles>c1ccc2c(-c3ccc4nnn(CC5CC5)c4c3)c[nH]c2c1</smiles>

1-(cyclopropylmethyl)-6-(1-(phenylsulfonyl)-1H-indol-3-yl)-1H-benzo[d][1,2,3]triazole was obtained after coupling 41 with 19d. Purified by flash chromatography (Cyclohexane/EtOAc 4:1). Then, 1(cyclopropylmethyl)-6-(1-(phenylsulfonyl)-1H-indol-3-yl)-1H-benzo[d][1,2,3]triazole was deprotected following general procedure $A$ to afford 19. Yield over two steps: $69 \%$. $\left.{ }^{1} \mathrm{H} \mathrm{NMR} \mathrm{(400MHz,DMSO}\right)$ : ? $0.44-0.67(4 \mathrm{H}, \mathrm{m}), 1.34-1.52(1 \mathrm{H}, \mathrm{m}), 4.67(2 \mathrm{H}, \mathrm{d}, \mathrm{J}=4.76 \mathrm{~Hz}), 7.10-7.28(2 \mathrm{H}, \mathrm{s}), 7.50(1 \mathrm{H}, \mathrm{d}, \mathrm{J}=5.72$ $\mathrm{Hz}), 7.77(1 \mathrm{H}, \mathrm{d}, \mathrm{J}=7.40 \mathrm{~Hz}), 7.88(1 \mathrm{H}, \mathrm{s}), 7.97-8.21(3 \mathrm{H}, \mathrm{m}), 11.54(1 \mathrm{H}, \mathrm{s}) .{ }^{13} \mathrm{C} \mathrm{NMR}(100 \mathrm{MHz}, \mathrm{DMSO}):$ ] 4.43; $11.80 ; 52.12 ; 106.89 ; 112.56 ; 115.67 ; 119.65 ; 120.42 ; 122.18 ; 124.58 ; 125.26 ; 125.35 ; 134.15$; 135.67; 137.47; 144.12. HRMS: $\mathrm{m} / \mathrm{z}(\mathrm{El}+)$, calculated for: $\mathrm{C}_{18} \mathrm{H}_{16} \mathrm{~N}_{4} 289.1409$ [M] $]^{+}$, found: 289.1449 [M] . Melting temp. range: $129-134^{\circ} \mathrm{C}$.

- 1-(cyclopropylmethyl)-5-(1H-indol-3-yl)-1H-benzo[d][1,2,3]triazole (20)<smiles>c1ccc2c(-c3ccc4c(c3)nnn4CC3CC3)c[nH]c2c1</smiles>

1-(cyclopropylmethyl)-5-(1-(phenylsulfonyl)-1H-indol-3-yl)-1H-benzo[d][1,2,3]triazole was obtained after coupling 41 with 20d. Purified by flash chromatography (Cyclohexane/EtOAc 4:1). Then, 1(cyclopropylmethyl)-5-(1-(phenylsulfonyl)-1H-indol-3-yl)-1H-benzo[d][1,2,3]triazole was deprotected following general procedure $A$ to afford 20 . Yield over two steps: $60 \% .{ }^{1} \mathrm{H}$ NMR (400MHz, DMSO): ?] $0.47-0.54(2 \mathrm{H}, \mathrm{m}), 0.54-0.63(2 \mathrm{H}, \mathrm{m}), 1.36-1.46(1 \mathrm{H}, \mathrm{m}), 4.63(2 \mathrm{H}, \mathrm{d}, \mathrm{J}=7.17 \mathrm{~Hz}), 7.14(1 \mathrm{H}, \mathrm{t}, \mathrm{J}=7.36$ $\mathrm{Hz}), 7.19(1 \mathrm{H}, \mathrm{t}, \mathrm{J}=7.44 \mathrm{~Hz}), 7.49(1 \mathrm{H}, \mathrm{d}, \mathrm{J}=7.83 \mathrm{~Hz}), 7.83(1 \mathrm{H}, \mathrm{d}, \mathrm{J}=2.36 \mathrm{~Hz}), 7.89-8.03(3 \mathrm{H}, \mathrm{m}), 8.23$ $(1 \mathrm{H}, \mathrm{s}), 11.46(1 \mathrm{H}, \mathrm{s}) .{ }^{13} \mathrm{C}$ NMR $(100 \mathrm{MHz}, \mathrm{DMSO})$ : $4.41 ; 11.81 ; 52.38 ; 111.52 ; 112.51 ; 115.35 ; 115.66$;

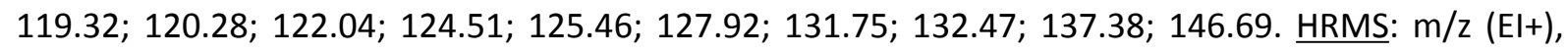
calculated for: $\mathrm{C}_{18} \mathrm{H}_{16} \mathrm{~N}_{4} 289.1409$ [M] ${ }^{+}$, found: 289.1447 [M] $]^{+}$. Melting temp. range: $206-213^{\circ} \mathrm{C}$.

- 2-(6-(1H-indol-3-yl)-1H-benzo[d][1,2,3]triazol-1-yl)-N,N-dimethylethan-1-amine (25) 
<smiles>CN(C)CCn1nnc2ccc(-c3c[nH]c4ccccc34)cc21</smiles>

2-(6-((1-(phenylsulfonyl)-1H-indol-3-yl)-1H-benzo[d][1,2,3]triazol-1-yl)- $N, N$-dimethylethan-1-amine was obtained after coupling 41 with 25d. Purified by flash chromatography (DCM/ $\mathrm{MeOH}_{0} \rightarrow 5 \%$ ). Then $\mathrm{N}, \mathrm{N}$-dimethyl-2-(6-(1-(phenylsulfonyl)-1H-indol-3-yl)-1H-benzo[d][1,2,3]triazol-1-yl)ethan-1-amine was deprotected following general procedure $A$ to afford 25. Yield over two steps: $50 \%$. $\underline{{ }^{1} \mathrm{H} \text { NMR }}$ (600MHz, DMSO): $2.22(6 \mathrm{H}, \mathrm{s}), 2.84(2 \mathrm{H}, \mathrm{s}), 4.86(2 \mathrm{H}, \mathrm{s}), 7.10-7.25(2 \mathrm{H}, \mathrm{m}), 7.43-7.55(1 \mathrm{H}, \mathrm{m}), 7.71$ $-7.80(1 \mathrm{H}, \mathrm{m}), 7.87(1 \mathrm{H}, \mathrm{s}), 7.98-8.06(2 \mathrm{H}, \mathrm{m}), 8.09(1 \mathrm{H}, \mathrm{s}), 11.55(1 \mathrm{H}, \mathrm{s}) .{ }^{13} \mathrm{C} \mathrm{NMR}(150 \mathrm{MHz}, \mathrm{DMSO}):$ 15.62; 46.04; 58.74; 107.06; 112.58; 115.71; 119.59; 120.39; 122.17; 124.52; 125.19; 125.40; 134.52; 135.53; 137.48; 144.03. HRMS: $\mathrm{m} / \mathrm{z}(\mathrm{El}+)$, calculated for: $\mathrm{C}_{18} \mathrm{H}_{19} \mathrm{~N}_{5} 306.1674[\mathrm{M}]^{+}$, found: 306.1716 [M] . Melting temp. range: $148-149^{\circ} \mathrm{C}$.

- 2-(5-(1H-indol-3-yl)-1H-benzo[d][1,2,3]triazol-1-yl)-N,N-dimethylethan-1-amine (26)<smiles>CN(C)CCn1nnc2cc(-c3c[nH]c4ccccc34)ccc21</smiles>

$\mathrm{N}, \mathrm{N}$-dimethyl-2-(5-(1-(phenylsulfonyl)-1H-indol-3-yl)-1H-benzo[d][1,2,3]triazol-1-yl)ethan-1-amine (AK-2-392-1) was obtained after coupling 41 with 26d. Purified by flash chromatography (DCM/ $\mathrm{MeOH}_{0}$ $\rightarrow 5 \%)$. Then, $\mathrm{N}, \mathrm{N}$-dimethyl-2-(5-(1-(phenylsulfonyl)-1H-indol-3-yl)-1H-benzo[d][1,2,3]triazol-1-yl)ethan1-amine was deprotected following general procedure $A$ to afford 26 . Yield over two steps: $43 \%$. ${ }^{1} \mathrm{H}$ NMR $\left(400 \mathrm{MHz}, \mathrm{CDCl}_{3}\right)$ : 国 $2.35(6 \mathrm{H}, \mathrm{s}), 2.95(2 \mathrm{H}, \mathrm{t}, \mathrm{J}=6.90 \mathrm{~Hz}), 4.77(2 \mathrm{H}, \mathrm{t}, \mathrm{J}=6.90 \mathrm{~Hz}), 7.23(1 \mathrm{H}, \mathrm{t}, \mathrm{J}=7.36$ $\mathrm{Hz}), 7.29(1 \mathrm{H}, \mathrm{d}, \mathrm{J}=7.12 \mathrm{~Hz}), 7.42(1 \mathrm{H}, \mathrm{s}), 7.47(1 \mathrm{H}, \mathrm{d}, \mathrm{J}=7.96 \mathrm{~Hz}), 7.61(1 \mathrm{H}, \mathrm{d}, \mathrm{J}=8.56 \mathrm{~Hz}), 7.82(1 \mathrm{H}, \mathrm{d}$, $\mathrm{J}=7.84 \mathrm{~Hz}), 7.97(1 \mathrm{H}, \mathrm{d}, \mathrm{J}=7.84 \mathrm{~Hz}), 8.30(1 \mathrm{H}, \mathrm{s}), 8.50(1 \mathrm{H}, \mathrm{bs}) .{ }^{13} \mathrm{C} \mathrm{NMR}\left(100 \mathrm{MHz}, \mathrm{CDCl}_{3}\right)$ : 回 45.64; 46.63; 58.46; 109.59; 111.57; 117.39; 117.68; 119.52; 120.58; 122.18; 122.64; 125.75; 128.29; 131.86; 132.03; 136.69; 146.92. $\mathrm{HRMS}: \mathrm{m} / \mathrm{z}(\mathrm{El}+)$, calculated for: $\mathrm{C}_{18} \mathrm{H}_{19} \mathrm{~N}_{5} 306.1674[\mathrm{M}]^{+}$, found: $306.1716[\mathrm{M}]^{+}$. Melting temp. range: $168-171^{\circ} \mathrm{C}$.

- 6-(1H-indol-3-yl)-1-((tetrahydrofuran-3-yl)methyl)-1H-benzo[d][1,2,3]triazole (27)<smiles>c1ccc2c(-c3ccc4nnn(CC5CCOC5)c4c3)c[nH]c2c1</smiles> 
6-(1-(phenylsulfonyl)-1H-indol-3-yl)-1-((tetrahydrofuran-3-yl)methyl)-1H-benzo[d][1,2,3]triazole was obtained after coupling $\mathbf{4 1}$ with $\mathbf{2 7 d}$. Purified by flash chromatography (Cyclohexane/EtOAc 2:1). Then,6-(1-(phenylsulfonyl)-1H-indol-3-yl)-1-((tetrahydrofuran-3-yl)methyl)-1H-benzo[d][1,2,3]triazole was deprotected following general procedure $A$ to afford 27. Yield over two steps: $40 \% . \mathrm{NMR}$ $\left(400 \mathrm{MHz}, \mathrm{CDCl}_{3}\right)$ : 回 $1.77-1.84(1 \mathrm{H}, \mathrm{m}), 2.06-2.13(1 \mathrm{H}, \mathrm{m}), 3.04-3.11(1 \mathrm{H}, \mathrm{m}), 3.71-3.75(1 \mathrm{H}, \mathrm{m})$, $3.79-3.85(2 \mathrm{H}, \mathrm{m}), 3.99-4.04(2 \mathrm{H}, \mathrm{m}), 4.64(4 \mathrm{H}, \mathrm{d}, \mathrm{J}=7.00 \mathrm{~Hz}), 7.24(1 \mathrm{H}, \mathrm{t}, \mathrm{J}=7.47 \mathrm{~Hz}), 7.29(1 \mathrm{H}, \mathrm{t}$, J=7.45 Hz), $7.45(1 \mathrm{H}, \mathrm{d}, \mathrm{J}=1.95 \mathrm{~Hz}), 7.48(1 \mathrm{H}, \mathrm{d}, \mathrm{J}=8.07 \mathrm{~Hz}), 7.59(1 \mathrm{H}, \mathrm{d}, \mathrm{J}=8.52 \mathrm{~Hz}), 7.84(1 \mathrm{H}, \mathrm{d}, \mathrm{J}=8.46$ $\mathrm{Hz}), 7.98(1 \mathrm{H}, \mathrm{d}, \mathrm{J}=7.92 \mathrm{~Hz}), 8.32(1 \mathrm{H}, \mathrm{s}), 8.40(1 \mathrm{H}, \mathrm{s}) .{ }^{13} \mathrm{C} \mathrm{NMR}\left(100 \mathrm{MHz}, \mathrm{CDCl}_{3}\right)$ : $29.86 ; 39.76 ; 50.51$; 67.61; 70.97; 109.21; 111.58; 117.53; 117.64; 119.50; 120.66; 122.18; 122.73; 125.73; 128.52; 131.98; 132.03; 136.69; 146.87. HRMS: $\mathrm{m} / \mathrm{z}(\mathrm{El}+)$, calculated for : $\mathrm{C}_{19} \mathrm{H}_{18} \mathrm{~N}_{4} \mathrm{O} 319.1514$ [M] ${ }^{+}$, found: 319.1551 $[\mathrm{M}]^{+}$. Melting temp. range: $216-218^{\circ} \mathrm{C}$.

- 5-(1H-indol-3-yl)-1-((tetrahydrofuran-3-yl)methyl)-1H-benzo[d][1,2,3]triazole (28)<smiles>c1ccc2c(-c3ccc4c(c3)nnn4CC3CCOC3)c[nH]c2c1</smiles>

5-(1H-indol-3-yl)-1-((tetrahydrofuran-3-yl)methyl)-1H-benzo[d][1,2,3]triazole was obtained after coupling 41 with 28d. Purified by flash chromatography (Cyclohexane/EtOAc $\rightarrow$ 2:1). Yield: $82 \%$. $\underline{1 H}$ $\operatorname{NMR}\left(400 \mathrm{MHz}, \mathrm{CDCl}_{3}\right)$ : $1.78-1.85(1 \mathrm{H}, \mathrm{m}), 2.06-2.13(1 \mathrm{H}, \mathrm{m}), 3.05-3.12(1 \mathrm{H}, \mathrm{m}), 3.73-3.77(1 \mathrm{H}$, $\mathrm{m}), 3.78-3.86(2 \mathrm{H}, \mathrm{m}), 4.01(1 \mathrm{H}, \mathrm{m}, \mathrm{J}=5.51 \mathrm{~Hz}), 4.65(2 \mathrm{H}, \mathrm{d}, \mathrm{J}=7.68 \mathrm{~Hz}), 7.24-7.28(1 \mathrm{H}, \mathrm{m}), 7.31(1 \mathrm{H}$, $\mathrm{t}, \mathrm{J}=7.44 \mathrm{~Hz}), 7.47-7.51(2 \mathrm{H}, \mathrm{m}), 7.71(1 \mathrm{H}, \mathrm{d}, \mathrm{J}=8.58 \mathrm{~Hz}), 7.75(1 \mathrm{H}, \mathrm{s}), 7.95(1 \mathrm{H}, \mathrm{d}, \mathrm{J}=7.92 \mathrm{~Hz}), 8.11(1 \mathrm{H}$, d, J=8.58 Hz), $8.48(1 \mathrm{H}, \mathrm{s}) .{ }^{13} \mathrm{C} \mathrm{NMR}\left(100 \mathrm{MHz}, \mathrm{CDCl}_{3}\right)$ : [29.88; 39.72; 50.35; 67.62; 70.99; 106.32; $111.74 ; 117.67 ; 119.39 ; 120.23 ; 120.85 ; 122.75 ; 122.91 ; 125.08 ; 125.58 ; 134.01 ; 135.60 ; 136.77$; 144.71.

5-(1H-indol-3-yl)-1-((tetrahydrofuran-3-yl)methyl)-1H-benzo[d][1,2,3]triazole was deprotected following general procedure $B$ to afford 28 . Yield: $55 \%$. ${ }^{1} \mathrm{H}$ NMR $(400 \mathrm{MHz}, \mathrm{DMSO})$ : ? $1.67-1.79(1 \mathrm{H}$, $\mathrm{m}), 1.92-2.02(1 \mathrm{H}, \mathrm{m}), 2.90-3.01(1 \mathrm{H}, \mathrm{m}), 3.55-3.62(1 \mathrm{H}, \mathrm{m}), 3.64-3.77(2 \mathrm{H}, \mathrm{m}), 3.81-3.89(1 \mathrm{H}$, m), $4.78(2 \mathrm{H}, \mathrm{d}, \mathrm{J}=7.41 \mathrm{~Hz}), 7.14-7.24(2 \mathrm{H}, \mathrm{m}), 7.50(1 \mathrm{H}, \mathrm{d}, \mathrm{J}=7.71 \mathrm{~Hz}), 7.79(1 \mathrm{H}, \mathrm{d}, \mathrm{J}=8.50 \mathrm{~Hz}), 7.88$ $(1 \mathrm{H}, \mathrm{s}), 8.05(1 \mathrm{H}, \mathrm{t}, \mathrm{J}=9.23 \mathrm{~Hz}), 8.12(1 \mathrm{H}, \mathrm{s}), 11.54(1 \mathrm{H}, \mathrm{s}) .{ }^{13} \mathrm{C}$ NMR $(100 \mathrm{MHz}, \mathrm{DMSO}):$ ? $29.91 ; 49.94$; $67.29 ; 70.61 ; 106.65 ; 112.55 ; 115.66 ; 119.71 ; 120.44 ; 122.19 ; 124.72 ; 125.34 ; 134.39 ; 135.91 ; 137.48$; 143.98. HRMS: $\mathrm{m} / \mathrm{z}(\mathrm{El}+)$, calculated for: $\mathrm{C}_{19} \mathrm{H}_{18} \mathrm{~N}_{4} \mathrm{O} 319.1514$ [M] ${ }^{+}$, found: 319.1551 [M] $]^{+}$. Melting temp. range: $158-162^{\circ} \mathrm{C}$.

- 6-(1H-indol-3-yl)-1-((tetrahydrofuran-2-yl)methyl)-1H-benzotriazole (29) 
<smiles>c1ccc2c(-c3ccc4nnn(CC5CCCO5)c4c3)c[nH]c2c1</smiles>

6-(1-(phenylsulfonyl)-1H-indol-3-yl)-1-((tetrahydrofuran-2-yl)methyl)-1H-benzo[d][1,2,3]triazole was obtained after coupling $\mathbf{4 1}$ with 29d. Purified by flash chromatography (Cyclohexane/EtOAc 2:1). Then, 6-(1-(phenylsulfonyl)-1H-indol-3-yl)-1-((tetrahydrofuran-2-yl)methyl)-1H-benzo[d][1,2,3]triazole was deprotected following general procedure $B$ to afford 29 . Yield over two steps: $35 \%$. ${ }^{1} \mathrm{H} \mathrm{NMR}(400 \mathrm{MHz}$, $\left.\mathrm{CDCl}_{3}\right)$ : $1.65-1.90(3 \mathrm{H}, \mathrm{m}), 2.04-2.13(1 \mathrm{H}, \mathrm{m}), 3.72-3.84(2 \mathrm{H}, \mathrm{m}), 4.43-4.50(1 \mathrm{H}, \mathrm{m}), 4.72(1 \mathrm{H}$, dd, J=14.46, $5.51 \mathrm{~Hz}), 4.84(1 \mathrm{H}, \mathrm{dd}, \mathrm{J}=14.46 \mathrm{~Hz}, 4.08 \mathrm{~Hz}), 7,23-7.31(2 \mathrm{H}, \mathrm{m}), 7.48-7.51(2 \mathrm{H}, \mathrm{m}), 7.67$ $(1 \mathrm{H}, \mathrm{d}, \mathrm{J}=4.71 \mathrm{~Hz}), 7.94(1 \mathrm{H}, \mathrm{s}), 8.01(1 \mathrm{H}, \mathrm{d} \mathrm{J}=7.72 \mathrm{~Hz}), 8.06(1 \mathrm{H}, \mathrm{d}, \mathrm{J}=8.62 \mathrm{~Hz}), 8.65(1 \mathrm{H}, \mathrm{br} \mathrm{s}) .{ }^{13} \mathrm{C} \mathrm{NMR}$ $\left(100 \mathrm{MHz}_{1} \mathrm{CDCl}_{3}\right)$ : 目 25.69; 28.80; 52.21; 68.60; 77.92; 107.85; 111.73; 117.69; 119.56; 119.72; 120.70 ; $122.72 ; 122.77 ; 124.86 ; 125.64 ; 134.60 ; 135.27 ; 136.80 ; 144.72$. HRMS: $\mathrm{m} / \mathrm{z}$ calculated for $\mathrm{C}_{19} \mathrm{H}_{18} \mathrm{~N}_{4} \mathrm{O}$ $319.1514[\mathrm{M}]^{+}$, found: $319.1550[\mathrm{M}]^{+}$. Melting temp. range: $173-178{ }^{\circ} \mathrm{C}$.

- 5-(1H-indol-3-yl)-1-((tetrahydrofuran-2-yl)methyl)-1H-benzotriazole (30)<smiles>c1ccc2c(-c3ccc4c(c3)nnn4CC3CCCO3)c[nH]c2c1</smiles>

5-(1-(phenylsulfonyl)-1H-indol-3-yl)-1-((tetrahydrofuran-2-yl)methyl)-1H-benzo[d][1,2,3]triazole was obtained after coupling $\mathbf{4 1}$ with 30d. Purified by flash chromatography (Cyclohexane/EtOAc 2:1). Yield: $85 \%$. ${ }^{1} \mathrm{H} \mathrm{NMR}\left(400 \mathrm{MHz}, \mathrm{CDCl}_{3}\right)$ : $1.65-1.93(3 \mathrm{H}, \mathrm{m}), 2.05-2.17(1 \mathrm{H}, \mathrm{m}), 3.70-3.82(2 \mathrm{H}, \mathrm{m}), 4.43(1 \mathrm{H}$, $\mathrm{m}, \mathrm{J}=4.61 \mathrm{~Hz}), 4.71(1 \mathrm{H}, \mathrm{dd}, \mathrm{J}=5.76,14.49 \mathrm{~Hz}), 4.85(1 \mathrm{H}, \mathrm{dd}, \mathrm{J}=3.74,14.49 \mathrm{~Hz}), 7.34(1 \mathrm{H}, \mathrm{t}, \mathrm{J}=7.53 \mathrm{~Hz})$, $7.41(1 \mathrm{H}, \mathrm{t}, \mathrm{J}=7.71 \mathrm{~Hz}), 7.48(1 \mathrm{H}, \mathrm{t}, \mathrm{J}=7.66 \mathrm{~Hz}), 7.54-7.63(1 \mathrm{H}, \mathrm{m}), 7.80(1 \mathrm{H}, \mathrm{s}), 7.84(1 \mathrm{H}, \mathrm{d}, \mathrm{J}=7.89 \mathrm{~Hz})$, $7.92(1 \mathrm{H}, \mathrm{s}), 7.96(1 \mathrm{H}, \mathrm{d}, \mathrm{J}=7.42 \mathrm{~Hz}), 8.07-8.14(2 \mathrm{H}, \mathrm{m})$.

5-(1-(phenylsulfonyl)-1H-indol-3-yl)-1-((tetrahydrofuran-2-yl)methyl)-1H-benzo[d][1,2,3]triazole was

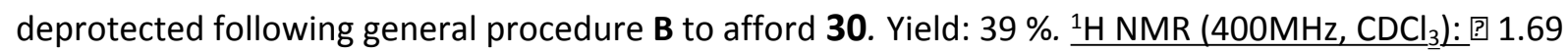
$-1.81(3 \mathrm{H}, \mathrm{m}), 1.99-2.08(1 \mathrm{H}, \mathrm{m}), 3.59-3.75(2 \mathrm{H}, \mathrm{m}), 4.30-4.40(1 \mathrm{H}, \mathrm{m}), 4.76(1 \mathrm{H}, \mathrm{dd} \mathrm{J}=14.43,6.82$ $\mathrm{Hz}), 4.84(1 \mathrm{H}, \mathrm{dd}, \mathrm{J}=14.43,3.75 \mathrm{~Hz}), 7.11-7.23(2 \mathrm{H}, \mathrm{m}), 7.48(1 \mathrm{H}, \mathrm{d}, \mathrm{J}=7.88 \mathrm{~Hz}), 7.81(1 \mathrm{H}, \mathrm{s}), 7.90-$ $7.98(3 \mathrm{H}, \mathrm{m}), 8.21(1 \mathrm{H}, \mathrm{s}), 11.45(1 \mathrm{H}, \mathrm{s}) .{ }^{13} \mathrm{C} \mathrm{NMR}\left(100 \mathrm{MHz} \mathrm{CDCl}_{3}\right)$ : 回 25.52; 28.81; 51.95; 67.92; 77.67; $112.01 ; 112.51 ; 115.16 ; 115.66 ; 119.33 ; 120.28 ; 122.05 ; 124.49 ; 125.45 ; 127.89 ; 132.43 ; 132.49$; 137.37; 146.53. HRMS: $m / z(E I+)$, calculated for: $\mathrm{C}_{19} \mathrm{H}_{18} \mathrm{~N}_{4} \mathrm{O} 319,15142[\mathrm{M}]^{+}$, found: $319,1550[\mathrm{M}]^{+}$. Melting temp. range: $205-216^{\circ} \mathrm{C}$. 
- 4-(2-(6-(1H-indol-3-yl)-1H-benzotriazol-1-yl)ethyl)morpholine (31)<smiles>c1ccc2c(-c3ccc4nnn(CCN5CCOCC5)c4c3)c[nH]c2c1</smiles>

4-(2-(6-(1-(phenylsulfonyl)-1H-indol-3-yl)-1H-benzo[d][1,2,3]triazol-1-yl)ethyl)morpholine was obtained after coupling $\mathbf{4 1}$ with 31d. Purified by flash chromatography (Cyclohexane/EtOAc $\rightarrow$ 2:1). Yield: $80 \%$. ${ }^{1 \mathrm{H} \mathrm{NMR}}\left(400 \mathrm{MHz}^{\mathrm{C}} \mathrm{CDCl}_{3}\right)$ : 回 $2.55-2.45(4 \mathrm{H}, \mathrm{m}), 2.93(2 \mathrm{H}, \mathrm{dt}, \mathrm{J}=13.2,6.7 \mathrm{~Hz}), 3.61(2 \mathrm{H}, \mathrm{d}$, $\mathrm{J}=4.0 \mathrm{~Hz}), 4.81-4.70(4 \mathrm{H}, \mathrm{m}), 7.30(2 \mathrm{H}, \mathrm{t}, \mathrm{J}=7.4 \mathrm{~Hz}), 7.39(1 \mathrm{H}, \mathrm{t}, \mathrm{J}=7.5 \mathrm{~Hz}), 7.45(2 \mathrm{H}, \mathrm{t}, \mathrm{J}=7.7 \mathrm{~Hz})$, $7.62-7.51(2 \mathrm{H}, \mathrm{m}), 7.78-7.72(2 \mathrm{H}, \mathrm{m}), 7.93(2 \mathrm{H}, \mathrm{d}, \mathrm{J}=7.5 \mathrm{~Hz}), 8.16-7.97(2 \mathrm{H}, \mathrm{m})$.

4-(2-(6-(1-(phenylsulfonyl)-1H-indol-3-yl)-1H-benzo[d][1,2,3]triazol-1-yl)ethyl)morpholine was deprotected following general procedure $A$ to afford 31. Yield: $42 \%{ }^{1} \mathrm{H} \mathrm{NMR}\left(400 \mathrm{MHz}_{1} \mathrm{CDCl}_{3}\right)$ : 2.48 $-2.58(4 \mathrm{H}, \mathrm{m}), 2.98(2 \mathrm{H}, \mathrm{t}, \mathrm{J}=6.53 \mathrm{~Hz}), 3.63-3.70(4 \mathrm{H}, \mathrm{m}), 4.78(2 \mathrm{H}, \mathrm{t}, \mathrm{J}=6.64 \mathrm{~Hz}), 7.23-7.30(2 \mathrm{H}, \mathrm{m}), 7.46-$ $7.51(2 \mathrm{H}, \mathrm{m}), 7.66(1 \mathrm{H}, \mathrm{d}, \mathrm{J}=8.50 \mathrm{~Hz}), 7.78(1 \mathrm{H}, \mathrm{s}), 7.95(1 \mathrm{H}, \mathrm{d}, \mathrm{J}=7.75 \mathrm{~Hz}), 8.07(1 \mathrm{H}, \mathrm{d}, \mathrm{J}=8.60 \mathrm{~Hz}), 8.92$

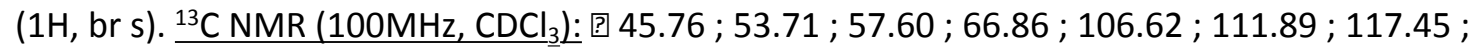
$119.33 ; 120.04 ; 120.70 ; 122.77 ; 122.92 ; 125.00 ; 125.55 ; 134.05 ; 135.48 ; 136.85 ; 144.69$. HRMS: $\mathrm{m} / \mathrm{z}(\mathrm{El}+)$, calculated for $\mathrm{C}_{20} \mathrm{H}_{21} \mathrm{~N}_{5} \mathrm{O}: 348,17797[\mathrm{M}]^{+}$, found: 348,1815 [M] $]^{+}$. Melting temp. range: 136 $-140^{\circ} \mathrm{C}$.

- 4-(2-(5-(1H-indol-3-yl)-1H-benzotriazol-1-yl)ethyl)morpholine (32)<smiles>c1ccc2c(-c3ccc4c(c3)nnn4CCN3CCOCC3)c[nH]c2c1</smiles>

4-(2-(5-(1-(phenylsulfonyl)-1H-indol-3-yl)-1H-benzo[d][1,2,3]triazol-1-yl)ethyl)morpholine was obtained after coupling $\mathbf{4 1}$ with $\mathbf{3 2 d}$. Purified by flash chromatography (Cyclohexane/EtOAc $\rightarrow$ 2:1). Quantitative yield. ${ }^{1} \mathrm{H} N M R\left(400 \mathrm{MHz}_{1} \mathrm{CDCl}_{3}\right): \delta 2.55-2.45(4 \mathrm{H}, \mathrm{m}), 2.93(2 \mathrm{H}, \mathrm{dt}, J=13.2,6.7 \mathrm{~Hz}), 3.61$ $(2 \mathrm{H}, \mathrm{d}, J=4.0 \mathrm{~Hz}), 4.81-4.70(4 \mathrm{H}, \mathrm{m}), 7.30(2 \mathrm{H}, \mathrm{t}, J=7.4 \mathrm{~Hz}), 7.39(1 \mathrm{H}, \mathrm{t}, J=7.5 \mathrm{~Hz}), 7.45(2 \mathrm{H}, \mathrm{t}, J=7.7$ $\mathrm{Hz}), 7.62-7.51(2 \mathrm{H}, \mathrm{m}), 7.78-7.72(2 \mathrm{H}, \mathrm{m}), 7.93(2 \mathrm{H}, \mathrm{d}, J=7.5 \mathrm{~Hz}), 8.16-7.97(2 \mathrm{H}, \mathrm{m})$.

4-(2-(5-(1-(phenylsulfonyl)-1H-indol-3-yl)-1H-benzo[d][1,2,3]triazol-1-yl)ethyl)morpholine deprotected following general procedure $A$ to afford 32 . Yield: $60 \%$. ${ }^{1 \mathrm{H} \mathrm{NMR}}\left(400 \mathrm{MHz}, \mathrm{CDCl}_{3}\right)$ : [? 2.51 $-2.60(4 \mathrm{H}, \mathrm{m}), 2.99(2 \mathrm{H}, \mathrm{t}, \mathrm{J}=6.29 \mathrm{~Hz}), 3.64-3.72(4 \mathrm{H}, \mathrm{m}), 4.78(2 \mathrm{H}, \mathrm{t}, \mathrm{J}=6.34 \mathrm{~Hz}), 7.19-7.32(2 \mathrm{H}, \mathrm{m})$, 7.43-7.50 (2H, m) ,7.61 (1H, d, J=8.43H), $7.83(1 \mathrm{H}, \mathrm{d}, \mathrm{J}=8.36 \mathrm{~Hz}), 7.97(1 \mathrm{H}, \mathrm{d}, \mathrm{J}=7.61 \mathrm{~Hz}), 8.30(1 \mathrm{H}, \mathrm{s}), 8.51$ $(1 \mathrm{H}, \mathrm{br} \mathrm{s}) .{ }^{13} \mathrm{C} \mathrm{NMR}\left(100 \mathrm{MHz}_{1} \mathrm{CDCl}_{3}\right)$ : $45.95 ; 53.69 ; 57.68 ; 66.90 ; 109.67 ; 111.62 ; 117.34 ; 117.59$; $119.49 ; 120.61 ; 122.23 ; 122.68 ; 125.71 ; 128.27 ; 131.93 ; 132.05 ; 136.70 ; 146.91$. HRMS: $\mathrm{m} / \mathrm{z}$ calculated for $\mathrm{C}_{20} \mathrm{H}_{21} \mathrm{~N}_{5} \mathrm{O}: 348,17797[\mathrm{M}]^{+}$, found: $348,1815[\mathrm{M}]^{+}$. Melting temp. range: $155-159{ }^{\circ} \mathrm{C}$. 
- 3-(6-(1H-indol-3-yl)-1H-benzo[d][1,2,3]triazol-1-yl)propane-1,2-diol (39)<smiles>OCC(O)Cn1nnc2ccc(-c3c[nH]c4ccccc34)cc21</smiles>

3-(6-((1-(phenylsulfonyl)-1H-indol-3-yl)-1H-benzo[d][1,2,3]triazol-1-yl)propane-1,2-diol was obtained after coupling 41 with 39d. Purified by flash chromatography (DCM/ $\left.\mathrm{MeOH}_{0 \rightarrow 5 \%}\right)$. Then, 3-(6-((1(phenylsulfonyl)-1H-indol-3-yl)-1H-benzo[d][1,2,3]triazol-1-yl)propane-1,2-diol was deprotected following general procedure $A$ to afford 39. Yield over two steps: $31 \% .{ }^{1} \mathrm{H} N \mathrm{NMR}(400 \mathrm{MHz}, \mathrm{MeOD})$ : [? $3.65(2 \mathrm{H}, \mathrm{d}, \mathrm{J}=5.05 \mathrm{~Hz}), 4.21(1 \mathrm{H}, \mathrm{bs}), 4.67-4.81(2 \mathrm{H}, \mathrm{m}), 7.07-7.31(2 \mathrm{H}, \mathrm{m}), 7.47(1 \mathrm{H}, \mathrm{d}, \mathrm{J}=7.77 \mathrm{~Hz})$, $7.58(1 \mathrm{H}, \mathrm{s}), 7.84-7.97(3 \mathrm{H}, \mathrm{m}), 8.19(1 \mathrm{H}, \mathrm{s})$. Labile protons not visible. ${ }^{13} \mathrm{C}$ NMR $(100 \mathrm{MHz}, \mathrm{MeOD})$ : ? 50.95; 63.35; 70.97; 110.79; 111.42; 114.58; 115.91; 118.51; 119.56; 121.55; 122.82; 125.40; 128.03; 132.52; 133.33; 137.33; 146.06. HRMS: $m / z(E l+)$, calculated for : $\mathrm{C}_{17} \mathrm{H}_{16} \mathrm{~N}_{4} \mathrm{O}_{2} 309.1307$ [M] ${ }^{+}$, found: $309.1346[\mathrm{M}]^{+}$. Melting temp. range: $65-73^{\circ} \mathrm{C}$.

- 3-(5-(1H-indol-3-yl)-1H-benzo[d][1,2,3]triazol-1-yl)propane-1,2-diol (40)<smiles>OCC(O)Cn1nnc2cc(-c3c[nH]c4ccccc34)ccc21</smiles>

3-(5-((1-(phenylsulfonyl)-1H-indol-3-yl)-1H-benzo[d][1,2,3]triazol-1-yl)propane-1,2-diol was obtained after coupling $\mathbf{4 1}$ with $\mathbf{4 0 d}$. Purified by flash chromatography (DCM/ $\left./ \mathrm{MeOH}_{0 \rightarrow 5 \%}\right)$. Then, 3-(5-((1(phenylsulfonyl)-1H-indol-3-yl)-1H-benzo[d][1,2,3]triazol-1-yl)propane-1,2-diol was deprotected following general procedure $A$ to afford 40 . Yield over two steps: $28 \%$. ${ }^{1 \mathrm{H} ~ N M R ~(600 M H z, ~ D M S O): ~ ?] ~}$ $3.40-3.52(2 \mathrm{H}, \mathrm{m}), 3.91-4.06(1 \mathrm{H}, \mathrm{m}), 4.62-4.72(1 \mathrm{H}, \mathrm{m}), 4.82-4.97(2 \mathrm{H}, \mathrm{m}), 5.20(1 \mathrm{H}, \mathrm{br} \mathrm{s}), 7.12$ - $7.24(2 \mathrm{H}, \mathrm{m}), 7.49(1 \mathrm{H}, \mathrm{d}, \mathrm{J}=7.19 \mathrm{~Hz}), 7.74(1 \mathrm{H}, \mathrm{d}, \mathrm{J}=7.80 \mathrm{~Hz}), 7.85(1 \mathrm{H}, \mathrm{s}), 8.03(2 \mathrm{H}, \mathrm{d}, \mathrm{J}=7.44 \mathrm{~Hz}), 8.09$ $(1 \mathrm{H}, \mathrm{s}), 11.51(1 \mathrm{H}, \mathrm{s}) .{ }^{13} \mathrm{C}$ NMR $(150 \mathrm{MHz}, \mathrm{DMSO}):$ ? $51.56 ; 63.84 ; 71.58 ; 107.71 ; 111.81 ; 112.56$; $115.79 ; 119.43 ; 119.64 ; 120.40 ; 122.18 ; 124.46 ; 125.06 ; 125.40 ; 135.20 ; 135.33 ; 137.49 ; 144.05$. HRMS: $\mathrm{m} / \mathrm{z}(\mathrm{El}+)$, calculated for : $\mathrm{C}_{17} \mathrm{H}_{16} \mathrm{~N}_{4} \mathrm{O}_{2} 309.1307[\mathrm{M}]^{+}$, found: 309.1346 [M] ${ }^{+}$. Melting temp. range: $59-65^{\circ} \mathrm{C}$.

2.9.2. List of compounds obtained following cross-coupling procedure $B$ A. using tert-butyl 6-fluoro-3-(4,4,5,5-tetramethyl-1,3,2-dioxaborolan-2-yl)-1H-indole-1carboxylate (42).

- 6-(6-fluoro-1H-indol-3-yl)-1-methyl-1H-benzotriazole (8a) 
<smiles>Cn1nnc2ccc(-c3c[nH]c4cc(F)ccc34)cc21</smiles>

tert-butyl 6-fluoro-3-(1-methyl-1H-benzo[d][1,2,3]triazol-6-yl)-1H-indole-1-carboxylate was obtained after coupling $\mathbf{4 2}$ with $\mathbf{8 b}$. Purified by flash chromatography (AcOEt/Cyclohexane 1:1). Yield: $65 \%$. $\underline{{ }^{1} \mathrm{H}}$ $\operatorname{NMR}(400 \mathrm{MHz}, \mathrm{CDCl} 3):$ ? $1.71(9 \mathrm{H}, \mathrm{s}), 4.36(3 \mathrm{H}, \mathrm{s}), 7.09(1 \mathrm{H}, \mathrm{t}, \mathrm{J}=8.77 \mathrm{~Hz}), 7.64(1 \mathrm{H}, \mathrm{d}, \mathrm{J}=8.55 \mathrm{~Hz}), 7.69$ $-7.82(3 \mathrm{H}, \mathrm{m}, \mathrm{J}=5.58 \mathrm{~Hz}), 7.98(1 \mathrm{H}, \mathrm{d}, \mathrm{J}=7.89 \mathrm{~Hz}), 8.14(1 \mathrm{H}, \mathrm{d}, \mathrm{J}=8.57 \mathrm{~Hz})$.

tert-butyl 6-fluoro-3-(1-methyl-1H-benzo[d][1,2,3]triazol-6-yl)-1H-indole-1-carboxylate was deprotected following general procedure $\mathrm{C}$ to afford 8a. Yield: Quantitative. ${ }^{1} \mathrm{H} \mathrm{NMR}(400 \mathrm{MHz}$, DMSO): $4.36(3 \mathrm{H}, \mathrm{s}), 7.02(1 \mathrm{H}, \mathrm{ddd}, \mathrm{J}=9.23, \mathrm{~J}=9.21, \mathrm{~J}=2.12, \mathrm{~J}=2.28 \mathrm{~Hz}), 7.27(1 \mathrm{H}, \mathrm{dd}, \mathrm{J}=7.89,1.98 \mathrm{~Hz})$, $7.75(1 \mathrm{H}, \mathrm{d}, \mathrm{J}=8.92 \mathrm{~Hz}), 7.87(1 \mathrm{H}, \mathrm{d}, \mathrm{J}=2,27 \mathrm{~Hz}), 8.03-8.07(3 \mathrm{H}, \mathrm{m}), 11.60(1 \mathrm{H}, \mathrm{s}) .{ }^{13} \mathrm{C} \mathrm{NMR}(100 \mathrm{MHz}$, DMSO): 34,$57 ; 98.46 ; 106.85 ; 108.78 ; 115.86 ; 119.64 ; 120.95 ; 122.24 ; 124.54 ; 125.78 ; 134.70$; 135.12; 137.41; 144.10; 161.66. $\underline{\text { HRMS: }} \mathrm{m} / \mathrm{z}(\mathrm{El}+)$, calculated for: $\mathrm{C}_{15} \mathrm{H}_{10} \mathrm{FN}_{4} \mathrm{Na}^{+} 2671041[\mathrm{M}+\mathrm{Na}$ ] 288.0782, found: $288.0894[\mathrm{M}+\mathrm{Na}]$ Melting temp. range: $236-240^{\circ} \mathrm{C}$.

- 6-(6-fluoro-1H-indol-3-yl)-1-(piperazin-1-ylmethyl)-1H-benzo[d][1,2,3]triazole (37a)<smiles>Fc1ccc2c(-c3ccc4nnn(CN5CCNCC5)c4c3)c[nH]c2c1</smiles>

tert-butyl 3-(1-((4-(tert-butoxycarbonyl)piperazin-1-yl)methyl)-1H-benzo[d][1,2,3]triazol-6-yl)-6fluoro-1H-indole-1-carboxylate was obtained after coupling 42 with $37 \mathrm{~d}$. Purified by flash chromatography (Cyclohexane/EtOAc 1:1). Then, it was deprotected using deprotection procedure $\mathbf{C}$ to afford 6-(6-fluoro-1H-indol-3-yl)-1-(piperazin-1-ylmethyl)-1H-benzo[d][1,2,3]triazole. Purified by flash chromatography (Cyclohexane/EtOAc 1:1 $\rightarrow$ 0:1). Yield over two steps: $18 \% .{ }^{1}$ H NMR $(600 \mathrm{MHz}$, $\left.\mathrm{CDCl}_{3}\right)$ : $2.36-2.43(3 \mathrm{H}, \mathrm{m}), 2.61(3 \mathrm{H}, \mathrm{t}, \mathrm{J}=4.59 \mathrm{~Hz}), 2.83(2 \mathrm{H}, \mathrm{t}, \mathrm{J}=6.39 \mathrm{~Hz}), 4.84(2 \mathrm{H}, \mathrm{t}, \mathrm{J}=6.39 \mathrm{~Hz}), 6.99$ $(1 \mathrm{H}, \mathrm{dt}, \mathrm{J}=4.14 \mathrm{~Hz}), 7.26(1 \mathrm{H}, \mathrm{dd}, \mathrm{J}=2.10,9.84 \mathrm{~Hz}), 7.81(1 \mathrm{H}, \mathrm{s}), 7.88(1 \mathrm{H}, \mathrm{dd}, \mathrm{J}=1.35,8.61 \mathrm{~Hz}), 7.90-$ $7.96(2 \mathrm{H}, \mathrm{m}), 8.20(1 \mathrm{H}, \mathrm{s}), 11.58(1 \mathrm{H}, \mathrm{s}) .{ }^{13} \mathrm{C} \mathrm{NMR}\left(150 \mathrm{MHz}, \mathrm{CDCl}_{3}\right)$ : (1C, d, J=25.36 Hz), $108.64(1 \mathrm{C}, d, J=24.25 \mathrm{~Hz}), 111.74 ; 115.44 ; 115.90 ; 120.51$ (1C, d, J=10.13 Hz), 122.40; 125.06; 127.68; 131.91; 132.29; 137.34 (1C, d, J=12.43 Hz); 146.52; 158.58; 160.14. HRMS: $\mathrm{m} / \mathrm{z}$ (El+), calculated for: $351.1689[\mathrm{M}]^{+}$, found: $351.1340[\mathrm{M}]^{+}$. Melting temp. range: $195-199{ }^{\circ} \mathrm{C}$.

B. Using tert-butyl 3-(4,4,5,5-tetramethyl-1,3,2-dioxaborolan-2-yl)-1H-indole-1-carboxylate

- 6-(1H-indol-3-yl)-1-(2,2,2-trifluoroethyl)-1H-benzo[d][1,2,3]triazole (17) 
<smiles>FC(F)(F)Cn1nnc2ccc(-c3c[nH]c4ccccc34)cc21</smiles>

tert-butyl 3-(1-(2,2,2-trifluoroethyl)-1H-benzo[d][1,2,3]triazol-6-yl)-1H-indole-1-carboxylate was obtained after coupling with 17d. Purified by flash chromatography (Cyclohexane/EtOAc 4:1 $\rightarrow$ 1:2).

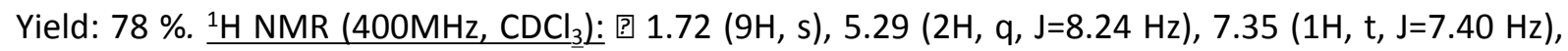
$7.42(1 \mathrm{H}, \mathrm{t}, \mathrm{J}=7.68 \mathrm{~Hz}), 7.73(1 \mathrm{H}, \mathrm{d}, \mathrm{J}=8.60 \mathrm{~Hz}), 7.77-7.86(3 \mathrm{H}, \mathrm{m}), 8.19(1 \mathrm{H}, \mathrm{d}, \mathrm{J}=8.60 \mathrm{~Hz}), 8.26(1 \mathrm{H}, \mathrm{d}$, $\mathrm{J}=7.76 \mathrm{~Hz})$.

tert-butyl 3-(1-(2,2,2-trifluoroethyl)-1H-benzo[d][1,2,3]triazol-6-yl)-1H-indole-1-carboxylate was deprotected using deprotection procedure $\mathbf{C}$ to afford 17. Purified by flash chromatography (Cyclohexane/EtOAc 4:1 $\rightarrow$ 1:2). Yield: $75 \%$. ${ }^{1} \mathrm{H} \mathrm{NMR}(400 \mathrm{MHz}, \mathrm{DMSO})$ : 国 $5.98(2 \mathrm{H}, \mathrm{q}, \mathrm{J}=9.12 \mathrm{~Hz}), 7.20$ $(2 \mathrm{H}$, quint, J=7.28 Hz), $7.51(1 \mathrm{H}, \mathrm{d}, \mathrm{J}=7.47 \mathrm{~Hz}), 7.85(1 \mathrm{H}, \mathrm{d}, \mathrm{J}=8.73 \mathrm{~Hz}), 7.91(1 \mathrm{H}, \mathrm{d}, \mathrm{J}=2.15 \mathrm{~Hz}), 8.08(1 \mathrm{H}$, d, J=7.62 Hz), $8.12(1 \mathrm{H}, \mathrm{d}, \mathrm{J}=8.71 \mathrm{~Hz}), 8.22(1 \mathrm{H}, \mathrm{s}), 11.60(1 \mathrm{H}, \mathrm{s}) .{ }^{13} \mathrm{C}$ NMR $(100 \mathrm{MHz}, \mathrm{DMSO}):$ ? 47.90 ; 48.24; 106.38; 112.63; 115.27; 119.70; 120.00; 120.56; 122.33; 125.19; 125.61; 134.93; 136.91; 137.52; 143.74. HRMS: $\mathrm{m} / \mathrm{z}(\mathrm{El}+)$, calculated for: $\mathrm{C}_{16} \mathrm{H}_{11} \mathrm{~F}_{3} \mathrm{~N}_{4} 317.0969[\mathrm{M}]^{+}$, found: $317.1006[\mathrm{M}]^{+}$. Melting temp. range: $171-172^{\circ} \mathrm{C}$.

- 6-(1H-indol-3-yl)-1-(2,2,2-trifluoroethyl)-1H-benzo[d][1,2,3]triazole (18)<smiles>FC(F)(F)Cn1nnc2cc(-c3c[nH]c4ccccc34)ccc21</smiles>

tert-butyl 3-(1-(2,2,2-trifluoroethyl)-1H-benzo[d][1,2,3]triazol-5-yl)-1H-indole-1-carboxylate was obtained after coupling with 18d. Purified by flash chromatography (Cyclohexane/EtOAc 4:1 $\rightarrow$ 1:2). Then, the coupling product was deprotected using deprotection procedure $\mathbf{C}$ to afford $\mathbf{1 8}$. Purified by

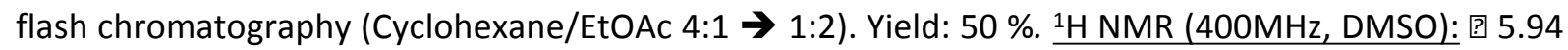
$(2 \mathrm{H}, \mathrm{q}, \mathrm{J}=9.08 \mathrm{~Hz}), 7.17(2 \mathrm{H}, \mathrm{td}, \mathrm{J}=7.34,20.39 \mathrm{~Hz}), 7.49(1 \mathrm{H}, \mathrm{d}, \mathrm{J}=7.85 \mathrm{~Hz}), 7.86(1 \mathrm{H}, \mathrm{d}, \mathrm{J}=1.90 \mathrm{~Hz}), 7.98$ $(2 \mathrm{H}, \mathrm{t}, \mathrm{J}=10.34 \mathrm{~Hz}), 8.03(2 \mathrm{H}, \mathrm{s}), 8.31(1 \mathrm{H}, \mathrm{s}), 11.50(1 \mathrm{H}, \mathrm{s}) .{ }^{13} \mathrm{C}$ NMR $(100 \mathrm{MHz}, \mathrm{DMSO})$ : 回 48.10 ; 48.44; $111.29 ; 112.54 ; 115.31 ; 115.54 ; 119.32 ; 120.37 ; 122.11 ; 124.78 ; 125.38 ; 129.07 ; 132.37 ; 133.28$; 137.38; 146.49. HRMS: $\mathrm{m} / z(\mathrm{El}+)$, calculated for: $\mathrm{C}_{16} \mathrm{H}_{11} \mathrm{~F}_{3} \mathrm{~N}_{4} 317.0969[\mathrm{M}]^{+}$, found: $317.1006[\mathrm{M}]^{+}$. Melting temp. range: $213-214^{\circ} \mathrm{C}$.

- 6-(1H-indol-3-yl)-1-(piperidin-4-ylmethyl)-1H-benzo[d][1,2,3]triazole (34) 
<smiles>c1ccc2c(-c3ccc4nnn(CC5CCNCC5)c4c3)c[nH]c2c1</smiles>

tert-butyl 3-(1-((1-(tert-butoxycarbonyl)piperidin-4-yl)methyl)-1H-benzo[d][1,2,3]triazol-6-yl)-1Hindole-1-carboxylate was obtained after coupling with 33d. Purified by flash chromatography

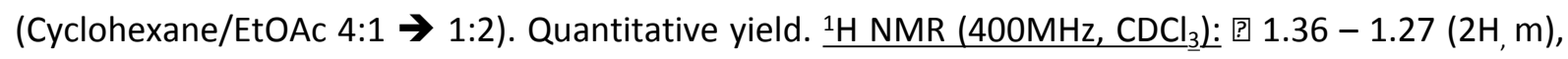
$1.43(9 \mathrm{H}, \mathrm{s}), 1.67(2 \mathrm{H}, \mathrm{s}), 1.72(9 \mathrm{H}, \mathrm{s}), 2.34-2.20(1 \mathrm{H}, \mathrm{m}), 2.66(2 \mathrm{H}, \mathrm{t}, J=12.3 \mathrm{~Hz}), 4.14(2 \mathrm{H}, \mathrm{s}), 4.55(2 \mathrm{H}$, $\mathrm{d}, J=7.0 \mathrm{~Hz}), 7.34(1 \mathrm{H}, \mathrm{t}, J=7.2 \mathrm{~Hz}), 7.42(1 \mathrm{H}, \mathrm{t}, J=7.4 \mathrm{~Hz}), 7.73-7.62(2 \mathrm{H}, \mathrm{m}), 7.81(2 \mathrm{H}, \mathrm{d}, J=7.4 \mathrm{~Hz})$, $8.14(1 \mathrm{H}, \mathrm{d}, J=8.5 \mathrm{~Hz}), 8.25(1 \mathrm{H}, \mathrm{d}, J=7.8 \mathrm{~Hz})$.

tert-butyl 3-(1-((1-(tert-butoxycarbonyl)piperidin-4-yl)methyl)-1H-benzo[d][1,2,3]triazol-6-yl)-1Hindole-1-carboxylate was deprotected following procedure $\mathbf{C}$ to afford 33. Purified by washing with diethylether. Quantitative yield. ${ }^{1} \mathrm{H}$ NMR $(400 \mathrm{MHz}, \mathrm{DMSO})$ : 回 $1.02-1.12(1 \mathrm{H}, \mathrm{m}), 1.45-1.58(2 \mathrm{H}, \mathrm{m})$, $1.72(2 \mathrm{H}, \mathrm{d}, \mathrm{J}=12.31 \mathrm{~Hz}), 2.25-2.39(2 \mathrm{H}, \mathrm{m}), 3.22(1 \mathrm{H}, \mathrm{d}, \mathrm{J}=10.72 \mathrm{~Hz}), 4.75(2 \mathrm{H}, \mathrm{d}, \mathrm{J}=4.50 \mathrm{~Hz}), 7.13-$ $7.24(2 \mathrm{H}, \mathrm{m}), 7.50(1 \mathrm{H}, \mathrm{d}, \mathrm{J}=6.69 \mathrm{~Hz}), 7.78(1 \mathrm{H}, \mathrm{d}, \mathrm{J}=8.02 \mathrm{~Hz}), 7.88(1 \mathrm{H}, \mathrm{s}), 7.99-8.14(3 \mathrm{H}, \mathrm{m}), 11.63$ $(1 \mathrm{H}, \mathrm{s}) .{ }^{13} \mathrm{C}$ NMR $(100 \mathrm{MHz}$, DMSO): 回 26.60; 34.72; 43.03; 52.04; 106.77; 112.59; 115.59; 119.71; $120.46 ; 122.17 ; 124.71 ; 125.33 ; 134.70 ; 135.96 ; 137.47 ; 143.91$. HRMS: $\mathrm{m} / \mathrm{z}(\mathrm{El}+)$, calculated for: $\mathrm{C}_{20} \mathrm{H}_{21} \mathrm{~N}_{5} 332.1831[\mathrm{M}]^{+}$, found: $332.1868[\mathrm{M}]^{+}$. Melting temp. range: $152-155^{\circ} \mathrm{C}$.

- 5-(1H-indol-3-yl)-1-(piperidin-4-ylmethyl)-1H-benzo[d][1,2,3]triazole (34)<smiles>c1ccc2c(-c3ccc4c(c3)nnn4CC3CCNCC3)c[nH]c2c1</smiles>

tert-butyl 3-(1-((1-(tert-butoxycarbonyl)piperidin-4-yl)methyl)-1H-benzo[d][1,2,3]triazol-5-yl)-1Hindole-1-carboxylate was obtained after coupling with $\mathbf{3 4 d}$. Purified by flash chromatography (Cyclohexane/EtOAc 1:0 $\rightarrow$ 1:1).Quantitative yield. ${ }^{1} \mathrm{H}$ NMR $\left(400 \mathrm{MHz}, \mathrm{CDCl}_{3}\right)$ : ? $1.36-1.27(2 \mathrm{H}, \mathrm{m})$, $1.43(9 \mathrm{H}, \mathrm{s}), 1.67(2 \mathrm{H}, \mathrm{s}), 1.72(9 \mathrm{H}, \mathrm{s}), 2.34-2.20(1 \mathrm{H}, \mathrm{m}), 2.66(2 \mathrm{H}, \mathrm{t}, J=12.3 \mathrm{~Hz}), 4.14(2 \mathrm{H}, \mathrm{s}), 4.55(2 \mathrm{H}$, $\mathrm{d}, J=7.0 \mathrm{~Hz}), 7.34(1 \mathrm{H}, \mathrm{t}, J=7.2 \mathrm{~Hz}), 7.42(1 \mathrm{H}, \mathrm{t}, J=7.4 \mathrm{~Hz}), 7.73-7.62(2 \mathrm{H}, \mathrm{m}), 7.81(2 \mathrm{H}, \mathrm{d}, J=7.4 \mathrm{~Hz})$, $8.14(1 \mathrm{H}, \mathrm{d}, J=8.5 \mathrm{~Hz}), 8.25(1 \mathrm{H}, \mathrm{d}, J=7.8 \mathrm{~Hz}), 8.33(1 \mathrm{H}, \mathrm{s})$.

tert-butyl 3-(1-((1-(tert-butoxycarbonyl)piperidin-4-yl)methyl)-1H-benzo[d][1,2,3]triazol-5-yl)-1Hindole-1-carboxylate was deprotected following procedure $\mathrm{C}$ to afford 34. Purified by washing with

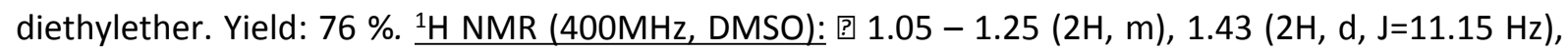
$1.98-2.13(1 \mathrm{H}, \mathrm{m}), 2.38(2 \mathrm{H}, \mathrm{t}, \mathrm{J}=11.55 \mathrm{~Hz}), 2.90(2 \mathrm{H}, \mathrm{d}, \mathrm{J}=11.05 \mathrm{~Hz}), 4.60(2 \mathrm{H}, \mathrm{d}, \mathrm{J}=6.48 \mathrm{~Hz}), 7.09-$ $7.23(2 \mathrm{H}, \mathrm{m}), 7.49(1 \mathrm{H}, \mathrm{d}, \mathrm{J}=7.68 \mathrm{~Hz}), 7.81(1 \mathrm{H}, \mathrm{s}), 7.88-8.01(3 \mathrm{H}, \mathrm{q}, \mathrm{J}=7.16 \mathrm{~Hz}), 8.22(1 \mathrm{H}, \mathrm{s}), 11.51(1 \mathrm{H}$, s). ${ }^{13} \mathrm{C} \mathrm{NMR}(100 \mathrm{MHz}, \mathrm{DMSO})$ : 回 $19.02 ; 26.60 ; 26.80 ; 34.71 ; 43.03 ; 52.04 ; 56.43 ; 56.52 ; 106.77 ; 112.55$; 
115.59; 119.72; 120.46; 122.18; 124.71; 125.33; 134.70; 135.96; 137.47; 143.91. HRMS: $m / z$ (EI+), calculated for: $\mathrm{C}_{20} \mathrm{H}_{21} \mathrm{~N}_{5} 332.1831[\mathrm{M}]^{+}$, found: $332.1868[\mathrm{M}]^{+}$. Melting temp. range: $215-219{ }^{\circ} \mathrm{C}$.

- 6-(1H-indol-3-yl)-1-(pyrrolidin-3-ylmethyl)-1H-benzo[d][1,2,3]triazole (35)<smiles>c1ccc2c(-c3ccc4nnn(CC5CCNC5)c4c3)c[nH]c2c1</smiles>

tert-butyl 3-(1-((1-(tert-butoxycarbonyl)pyrrolidin-3-yl)methyl)-1H-benzo[d][1,2,3]triazol-6-yl)-1Hindole-1-carboxylate was obtained after coupling with $35 \mathrm{~d}$. Purified by flash chromatography

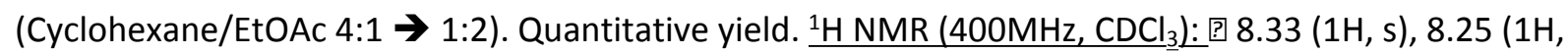
$\mathrm{d}, J=7.2 \mathrm{~Hz}), 7.82(2 \mathrm{H}, \mathrm{dd}, J=20.2,8.0 \mathrm{~Hz}), 7.60(1 \mathrm{H}, \mathrm{d}, J=8.5 \mathrm{~Hz}), 7.37(2 \mathrm{H}, \mathrm{dt}, J=30.0,7.3 \mathrm{~Hz}), 4.68$ $(1 \mathrm{H}, \mathrm{s}), 3.34(7 \mathrm{H}, \mathrm{m}), 1.71(9 \mathrm{H}, \mathrm{s}), 1.61(2 \mathrm{H}, \mathrm{s}), 1.46(9 \mathrm{H}, \mathrm{s})$.

tert-butyl 3-(1-((1-(tert-butoxycarbonyl)pyrrolidin-3-yl)methyl)-1H-benzo[d][1,2,3]triazol-6-yl)-1Hindole-1-carboxylate was deprotected following procedure $\mathrm{C}$ to afford 35 . Purified by washing with diethylether. Yield: $85 \%$. ${ }^{1} \mathrm{H}$ NMR (400MHz, DMSO): [ $1.54-1.75(1 \mathrm{H}, \mathrm{m}), 1.85-2.04(1 \mathrm{H}, \mathrm{m}), 2.80-$ $3.09(3 \mathrm{H}, \mathrm{m}), 3.17(2 \mathrm{H}, \mathrm{s}), 4.87(2 \mathrm{H}, \mathrm{s}), 7.18(2 \mathrm{H}, \mathrm{t}, \mathrm{J}=8.29 \mathrm{~Hz}), 7.50(2 \mathrm{H}, \mathrm{d}, \mathrm{J}=7.34 \mathrm{~Hz}), 7.79(2 \mathrm{H}, \mathrm{d}, \mathrm{J}=8.36$ $\mathrm{Hz}), 7.90(1 \mathrm{H}, \mathrm{s}), 8.00-8.12(2 \mathrm{H}, \mathrm{m}), 8.17(1 \mathrm{H}, \mathrm{s}), 11.65(1 \mathrm{H}, \mathrm{s}) .{ }^{13} \mathrm{C} N M R(100 \mathrm{MHz}, \mathrm{DMSO}):$ ? 26.80; 28.63; 38.90; 45.00; 48.52; 49.49; 106.61; 112.58; 115.56; 119.74; 120.45; 122.16; 124.75; 125.30; 125.37; 134.48; 136.04; 137.47; 143.89. HRMS: $\mathrm{m} / \mathrm{z}(\mathrm{El}+)$, calculated for : $\mathrm{C}_{19} \mathrm{H}_{19} \mathrm{~N}_{5} 318.1674$ [M] $]^{+}$, found: $318.1713[\mathrm{M}]^{+}$. Melting temp. range: $72-82^{\circ} \mathrm{C}$.

- 5-(1H-indol-3-yl)-1-(pyrrolidin-3-ylmethyl)-1H-benzo[d][1,2,3]triazole (36)<smiles>c1ccc2c(-c3ccc4c(c3)nnn4CC3CCNC3)c[nH]c2c1</smiles>

tert-butyl 3-(1-((1-(tert-butoxycarbonyl)pyrrolidin-3-yl)methyl)-1H-benzo[d][1,2,3]triazol-5-yl)-1H-

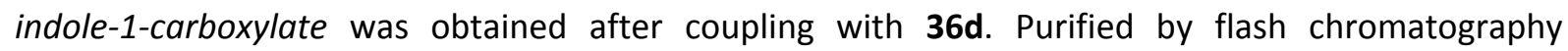
(Cyclohexane/EtOAc 1:0 $\rightarrow$ 1:1). Yield: $80 \%$. ${ }^{1} \mathrm{H} \mathrm{NMR}\left(400 \mathrm{MHz}, \mathrm{CDCl}_{3}\right)$ : $1.71(9 \mathrm{H}, \mathrm{s}), 2.91-3.05(1 \mathrm{H}$, $\mathrm{m}), 3.18-3.31(1 \mathrm{H}, \mathrm{m}), 3.31-3.45(1 \mathrm{H}, \mathrm{m}), 3.45-3.65(2 \mathrm{H}, \mathrm{m}), 4.64-4.72(2 \mathrm{H}, \mathrm{m}), 7.33(1 \mathrm{H}, \mathrm{t}, \mathrm{J}=7.36$ $\mathrm{Hz}), 7.41(1 \mathrm{H}, \mathrm{t}, \mathrm{J}=7.55 \mathrm{~Hz}), 7.60(1 \mathrm{H}, \mathrm{d}, \mathrm{J}=8.52 \mathrm{~Hz}), 7.77-7.82(2 \mathrm{H}, \mathrm{m}), 7.85(1 \mathrm{H}, \mathrm{d}, \mathrm{J}=7.81 \mathrm{~Hz}), 8.22-$ $8.29(1 \mathrm{H}, \mathrm{m}), 8.33(1 \mathrm{H}, \mathrm{s})$.

tert-butyl 3-(1-((1-(tert-butoxycarbonyl)pyrrolidin-3-yl)methyl)-1H-benzo[d][1,2,3]triazol-5-yl)-1Hindole-1-carboxylate was deprotected following procedure $\mathbf{C}$ to afford 36. Purified by washing with diethylether. Quantitative yield. ${ }^{1} \mathrm{H}$ NMR $(400 \mathrm{MHz}, \mathrm{DMSO})$ : ? $1.01-1.11(1 \mathrm{H}, \mathrm{m}), 2.79-2.88(2 \mathrm{H}, \mathrm{m})$, $3.30-3.46$ (multiplet under $\mathrm{H}_{2} \mathrm{O}$ signal), $4.80-4.96(2 \mathrm{H}, \mathrm{m}), 7.11-7.25(2 \mathrm{H}, \mathrm{m}), 7.49(1 \mathrm{H}, \mathrm{d}, \mathrm{J}=7.64$ 
$\mathrm{Hz}), 7.75(1 \mathrm{H}, \mathrm{d}, \mathrm{J}=8.46 \mathrm{~Hz}), 7.86(1 \mathrm{H}, \mathrm{s}), 7.99-8.07(2 \mathrm{H}, \mathrm{m}), 8.08(1 \mathrm{H}, \mathrm{s}), 11.53(1 \mathrm{H}, \mathrm{s}) .{ }^{13} \mathrm{C} \mathrm{NMR}$ (100MHz, DMSO): 26.81; 45.44; 45.98; 54.63; 58.36; 65.39; 107.03; 112.57; 115.70; 119.57; 119.68;

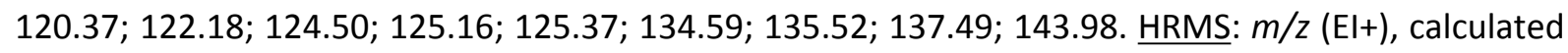
for : $\mathrm{C}_{19} \mathrm{H}_{19} \mathrm{~N}_{5} 318.1674[\mathrm{M}]^{+}$, found: $318.1711[\mathrm{M}]^{+}$. Melting temp. range: $179-182^{\circ} \mathrm{C}$.

- 6-(1H-indol-3-yl)-1-(2-(piperazin-1-yl)ethyl)-1H-benzo[d][1,2,3]triazole (37)<smiles>c1ccc2c(-c3ccc4nnn(CCN5CCNCC5)c4c3)c[nH]c2c1</smiles>

tert-butyl 3-(1-((1-(tert-butoxycarbonyl)piperidin-4-yl)methyl)-1H-benzo[d][1,2,3]triazol-6-yl)-1Hindole-1-carboxylate was obtained after coupling with 37d. Purified by flash chromatography (Cyclohexane/EtOAc 1:1). Yield: $85 \%$. ${ }^{1 \mathrm{H} N M R}(400 \mathrm{MHz}, \mathrm{DMSO}): 2.35-2.44(3 \mathrm{H}, \mathrm{m}), 2.58-2.65(3 \mathrm{H}$, m), $2.83(2 \mathrm{H}, \mathrm{t}, \mathrm{J}=6.21 \mathrm{~Hz}), 4.84(2 \mathrm{H}, \mathrm{t}, \mathrm{J}=6.20 \mathrm{~Hz}), 7.14(2 \mathrm{H}, \mathrm{t}, \mathrm{J}=7.08 \mathrm{~Hz}), 7.19(2 \mathrm{H}, \mathrm{t}, \mathrm{J}=7.47 \mathrm{~Hz}), 7.49$ $(2 \mathrm{H}, \mathrm{d}, \mathrm{J}=7.93 \mathrm{~Hz}), 7.83(2 \mathrm{H}, \mathrm{d}, \mathrm{J}=1.54 \mathrm{~Hz}), 7.89-7.98(3 \mathrm{H}, \mathrm{m}), 8.22(2 \mathrm{H}, \mathrm{s}), 11.50(1 \mathrm{H}, \mathrm{s}) .{ }^{13} \mathrm{C} \mathrm{NMR}$ (100MHz, DMSO): 45.65; 46.01; 54.59; 58.23; 111.66; 112.52; 115.28; 115.66; 119.32; 120.26; $122.02 ; 124.49 ; 125.46 ; 127.73 ; 132.16 ; 132.41 ; 137.40 ; 146.56$.

tert-butyl 3-(1-((1-(tert-butoxycarbonyl)piperidin-4-yl)methyl)-1H-benzo[d][1,2,3]triazol-6-yl)-1Hindole-1-carboxylate was deprotected using deprotection procedure $\mathrm{C}$ to afford 37 . Purified by washing with diethylether. Quantitative yield. ${ }^{1} \mathrm{H} \mathrm{NMR}(400 \mathrm{MHz}, \mathrm{DMSO})$ : $2.36-2.46(4 \mathrm{H}, \mathrm{m}), 2.56$ $-2.64(4 \mathrm{H}, \mathrm{m}), 2.81-2.90(2 \mathrm{H}, \mathrm{m}), 4.85-4.93(2 \mathrm{H}, \mathrm{m}), 7.14-7.24(2 \mathrm{H}, \mathrm{m}), 7.50(1 \mathrm{H}, \mathrm{d}, \mathrm{J}=7.68 \mathrm{~Hz})$, $7.77(1 \mathrm{H}, \mathrm{d}, \mathrm{J}=8.56 \mathrm{~Hz}), 7.88(1 \mathrm{H}, \mathrm{s}), 8.01-8.08(2 \mathrm{H}, \mathrm{m}), 8.10(1 \mathrm{H}, \mathrm{s}), 11.55(1 \mathrm{H}, \mathrm{s}) .{ }^{13} \mathrm{C} \mathrm{NMR}(100 \mathrm{MHz}$, DMSO): 回 45.65; 46.01; 54.59; 58.23; 111.66; 112.52; 115.28; 115.66; 119.32; 120.26; 122.02; 124.49; $125.46 ; 127.73 ; 132.16 ; 132.41 ; 137.40 ; 146.56$. HRMS: $\mathrm{m} / \mathrm{z}(\mathrm{El}+)$, calculated for: $\mathrm{C}_{20} \mathrm{H}_{22} \mathrm{~N}_{6} 347.1939$ $[\mathrm{M}]^{+}$, found: $347.1978[\mathrm{M}]^{+}$. Melting temp. range: $44-46^{\circ} \mathrm{C}$.

- 5-(1H-indol-3-yl)-1-(piperazin-4-yl)ethyl)-1H-benzo[d][1,2,3]triazole (38)<smiles>c1ccc2c(-c3ccc4c(c3)nnn4CCN3CCNCC3)c[nH]c2c1</smiles>

tert-butyl 3-(1-((4-(tert-butoxycarbonyl)piperazin-1-yl)methyl)-1H-benzo[d][1,2,3]triazol-5-yl)-1Hindole-1-carboxylate was obtained after coupling with 38d. Purified by flash chromatography (Cyclohexane/EtOAc 1:1). Yield: $80 \%$ \% ${ }^{1} \mathrm{H}$ NMR $\left(400 \mathrm{MHz}_{1} \mathrm{CDCl}_{3}\right)$ : 国 $2.43-2.55(4 \mathrm{H}, \mathrm{m}), 3.00(1 \mathrm{H}, \mathrm{t}$, $\mathrm{J}=6.50 \mathrm{~Hz}), 3.35-3.44(4 \mathrm{H}, \mathrm{m}), 4.80(2 \mathrm{H}, \mathrm{t}, \mathrm{J}=6.49 \mathrm{~Hz}), 7.33(1 \mathrm{H}, \mathrm{t}, \mathrm{J}=7.49 \mathrm{~Hz}), 7.40(1 \mathrm{H}, \mathrm{t}, \mathrm{J}=7.70 \mathrm{~Hz})$, $7.64(1 \mathrm{H}, \mathrm{d}, \mathrm{J}=8.55 \mathrm{~Hz}), 7.75-7.82(2 \mathrm{H}, \mathrm{m}), 7.85(1 \mathrm{H}, \mathrm{d}, \mathrm{J}=7.79 \mathrm{~Hz}), 8.20-8.29(1 \mathrm{H}, \mathrm{m}), 8.31(1 \mathrm{H}, \mathrm{s})$. 
tert-butyl 3-(1-((4-(tert-butoxycarbonyl)piperazin-1-yl)methyl)-1H-benzo[d][1,2,3]triazol-5-yl)-1Hindole-1-carboxylate was deprotected using deprotection procedure $\mathbf{C}$ to afford 38 . Purified by washing with diethylether. Quantitative yield. ${ }^{1} \mathrm{H} \mathrm{NMR}(400 \mathrm{MHz}, \mathrm{DMSO})$ : ? $2.41-2.49(3 \mathrm{H}, \mathrm{m}), 2.61-$ $2.67(3 \mathrm{H}, \mathrm{m}), 2.87(2 \mathrm{H}, \mathrm{t}, \mathrm{J}=6.16 \mathrm{~Hz}), 4.89(2 \mathrm{H}, \mathrm{t}, \mathrm{J}=6.17 \mathrm{~Hz}), 7.16(1 \mathrm{H}, \mathrm{t}, \mathrm{J}=7.14 \mathrm{~Hz}), 7.21(1 \mathrm{H}, \mathrm{t}, \mathrm{J}=7.45$ $\mathrm{Hz}), 7.50(1 \mathrm{H}, \mathrm{d}, \mathrm{J}=7.78 \mathrm{~Hz}), 7.77(1 \mathrm{H}, \mathrm{d}, \mathrm{J}=8.68 \mathrm{~Hz}), 7.88(1 \mathrm{H}, \mathrm{d}, \mathrm{J}=2.32 \mathrm{~Hz}), 8.01-8.07(2 \mathrm{H}, \mathrm{m}), 8.10$ $(1 \mathrm{H}, \mathrm{s}) .{ }^{13} \mathrm{C}$ NMR $(100 \mathrm{MHz}$, DMSO): 回 31.01; 37.72; 46.04; 53.58; 111.47; 112.53; 115.34; 115.63; $119.30 ; 120.26 ; 122.01 ; 124.51 ; 125.45 ; 127.96 ; 132.30 ; 132.47 ; 137.39 ; 146.47$. HRMS: $m / z$ (EI+), calculated for: $\mathrm{C}_{20} \mathrm{H}_{22} \mathrm{~N}_{6} 347.1939[\mathrm{M}]^{+}$, found: $347.1979[\mathrm{M}]^{+}$. Melting temp. range: $177-185^{\circ} \mathrm{C}$. 